$\underline{\text { Preprint typeset in JHEP style - PAPER VERSION }}$

hep-th/0607227

LPTENS-06-27

\title{
Lectures* on Black Holes, Topological Strings and Quantum Attractors
}

\author{
Boris Pioline \\ LPTHE, Universités Pierre et Marie Curie (Paris 6) et Denis Diderot (Paris 7), \\ 4 place Jussieu, 75252 Paris cedex 05, France \\ LPTENS, Département de Physique de l'ENS, 24 rue Lhomond \\ 75231 Paris cedex 05, France \\ E-mail: pioline@lpthe.jussieu.fr
}

\begin{abstract}
In these lecture notes, we review some recent developments on the relation between the macroscopic entropy of four-dimensional BPS black holes and the microscopic counting of states, beyond the thermodynamical, large charge limit. After a brief overview of charged black holes in supergravity and string theory, we give an extensive introduction to special and very special geometry, attractor flows and topological string theory, including holomorphic anomalies. We then expose the Ooguri-VerlindeVafa (OSV) conjecture which relates microscopic degeneracies to the topological string amplitude, and review precision tests of this formula on "small" black holes. Finally, motivated by a holographic interpretation of the OSV conjecture, we discuss the radial quantization of BPS black holes (i.e. quantum attractors) and present a recent conjecture relating exact black hole degeneracies to Fourier coefficients of certain automorphic forms.
\end{abstract}

${ }^{*}$ Lectures delivered at the RTN Winter School on Strings, Supergravity and Gauge theories, (CERN, January 16-20, 2006), the 11-th APCTP/KIAS String Winter School (Pohang, Feb 8-15 2005) and the Winter School on the Attractor Mechanism (Frascati, March 20-24, 2006). 


\section{Contents}

1. Introduction $\quad 2$

2. Extremal Black Holes in String Theory $\quad 4$

2.1 Black Hole Thermodynamics 4

2.2 Extremal Reissner-Nordström Black Holes 7

2.3 Black Hole Counting via D-branes 8

2.4 Counting $\mathcal{N}=4$ Dyons via Automorphic Forms 10

3. Special Geometry And Black Hole Attractors 12

$3.1 \mathcal{N}=2$ SUGRA and Special Geometry 13

$3.2 \mathcal{N}=2$ SUGRA and String Theory 16

3.3 Attractor Flows and Bekenstein-Hawking Entropy 18

3.4 Bekenstein-Hawking entropy and Legendre transform 20

3.5 Very Special Supergravities and Jordan Algebras 22

3.6 Bekenstein-Hawking Entropy in Very Special Supergravities 25

4. Topological String Primer $\quad 29$

4.1 Topological Sigma Models 29

4.1.1 Topological A-model 31

4.1.2 Topological B-model 32

4.2 Topological Strings 33

4.3 Gromov-Witten, Gopakumar-Vafa and Donaldson-Thomas Invariants 34

4.4 Holomorphic Anomalies and the Wave Function Property 37

5. Higher Derivative Corrections and Topological Strings 41

5.1 Gravitational F-terms and Topological Strings 41

5.2 Bekenstein-Hawking-Wald Entropy 44

5.3 The Ooguri-Strominger-Vafa Conjecture 45

6. Precision Counting of Small Black Holes 48

6.1 Degeneracies of DH states and the Rademacher formula 48

6.2 Macroscopic entropy and the topological amplitude $\quad 51$

6.3 Testing the OSV Formula 52

$6.4 \mathcal{N}=2$ Orbifolds $\quad 54$ 
7. Quantum Attractors and Automorphic Partition Functions 55

7.1 OSV Conjecture and Quantum Attractors 55

7.2 Attractor Flows and Geodesic Motion 58

$\begin{array}{lll}7.2 .1 & \text { Conserved charges } & 60\end{array}$

7.2.2 Extremal black holes and light-like geodesics 61

7.2.3 BPS Black Holes and BPS Geodesics 62

7.2.4 Radial flow in $\mathcal{N}=8$ and $\mathcal{N}=4$ SUGRA 62

7.2.5 Attractor Flow in $\mathcal{N}=2$ SUGRA and the $c^{*}$-map 63

7.2.6 The Universal Sector 66

7.3 Quantum Attractors 68

7.3.1 BPS Hilbert space and twistors 69

7.4 Very Special Quantum Attractors $\quad 70$

$\begin{array}{ll}\text { 7.4.1 Quasiconformal Action and Twistor Space } & 70\end{array}$

7.4.2 Embedding $H_{B P S}$ in $H$ and the Spherical Vector 73

$\begin{array}{lll}\text { 7.4.3 The Minimal Representation } & 73\end{array}$

$\begin{array}{lll}7.5 & \text { Speculations } & 74\end{array}$

7.5.1 The Generalized Topological String Amplitude 74

$\begin{array}{ll}\text { 7.5.2 Automorphic Partition Functions } & 76\end{array}$

$\begin{array}{ll}\text { 8. Conclusion } & 77\end{array}$

\section{Introduction}

Once regarded as unphysical solutions of General Relativity, black holes now occupy the central stage, both in astrophysics, with mounting evidence of stellar size and supermassive black holes in binary systems and in galactic centers, and in theoretical particle physics, where they plausibly dominate the high energy behavior of quantum gravity (see e.g. [1] and [2] for recent accounts).

In this respect, the Bekenstein-Hawking entropy of black holes is one of the very few hints in our hands about the nature of quantum gravity: just as the macroscopic thermodynamical properties of perfect gases hinted at their microscopic atomistic structure, the classical thermodynamical properties of black holes suggest the existence of quantized micro-states, whose dynamics would account for the macroscopic production of entropy. 
One of the great successes of string theory is to have made this idea precise, albeit for black holes which have little to do with reality: non-rotating, extremal, supersymmetric, charged black holes can indeed be viewed as bound states of D-branes and other extended objects, whose microscopic "open-string" fluctuations account for the macroscopic Bekenstein-Hawking entropy. (More generally, in the spirit of the AdS/CFT correspondence, the macroscopic gravitational dynamics should be describable by microscopic "gauge theoretical" degrees of freedom). The agreement is quantitatively exact in the "thermodynamical" limit of large charge, where the counting of the degrees of freedom requires only a gross understanding of their dynamics.

While the prospects of carrying this quantitative agreement over to more realistic black holes remain distant, it is interesting to investigate whether the already remarkable agreement found for supersymmetric extremal black holes can be pushed beyond the thermodynamical limit. Indeed, this regime in principle allows to probe quantumgravity corrections to the low energy Einstein-Maxwell Lagrangian, while testing our description of the microscopic degrees of freedom in greater detail.

The aim of these lectures is to describe some recent developments in this direction, in the context of BPS black holes of $\mathcal{N} \geq 2$ supergravity.

In Section 2, we give an overview of extremal Reissner-Nordström black holes, recall their string theory counting to leading order, and discuss an early proposal to relate the exact microscopic degeneracies to Fourier coefficients of a certain modular form.

In Section 3, we recall the essentials of special geometry, and describe the "attractor flow", which governs the radial evolution of the scalar fields and determines the horizon geometry in terms of asymptotic charges. We illustrate these results in the context of "very special supergravities", which enjoy special symmetries properties.

In Section 4, we give a self-contained introduction to topological string theory, which allows to compute an infinite set of higher-derivative "F-term" corrections in the low energy Lagrangian. We emphasize the wave function interpretation of the holomorphic anomaly, which underlies much of the subsequent developments.

In Section 5, we discuss the effects of these "F-term" corrections on the macroscopic entropy, and formulate the Ooguri-Strominger-Vafa (OSV) conjecture, which relates these macroscopic corrections to the micro-canonical counting.

In Section 6, we submit this conjecture to a precision test, in the context of "small black holes": these are dual to perturbative heterotic states, and can therefore be counted exactly using standard conformal field theory techniques.

Finally, in Section 7, motivated by a holographic re-interpretation of the OSV conjecture, we consider the problem of "quantizing the attractor flow". In this framework, we find evidence for a one-parameter generalization of the usual topological string amplitude, and conjecture a relation between black hole degeneracies and Fourier co- 
efficients of certain automorphic forms. These are analogous, yet different from the genus-2 modular forms discussed in Section 2.4, and it is hoped that they will allow for an exact counting of BPS black holes at finite values of the charges.

We have included a number of exercices, most of which are quite easy, which are intended to illustrate, complement or extend the discussion in the main text. The dedicated student might learn more from solving the exercices than from pondering over the text.

\section{Extremal Black Holes in String Theory}

In this section, we give a general overview of extremal black holes in Einstein-Maxwell theory, comment on their embedding in string theory, and outline their microscopic description as bound states of D-branes. We also review an early conjecture that relates the exact microscopic degeneracies of BPS black holes to Fourier coefficients of a certain modular form. We occasionally make use of notions that will be explained in later Sections. For a general introduction to black hole thermodynamics, the reader may consult e.g. $[3,4]$.

\subsection{Black Hole Thermodynamics}

Our starting point is the Einstein-Maxwell Lagrangian for gravity and a massless Abelian gauge field in $3+1$ dimensions,

$$
S=\int d^{4} x \frac{1}{16 \pi G}\left[\sqrt{-g} R-\frac{1}{4} F \wedge * F\right]
$$

Assuming staticity and spherical symmetry, the only solution with electric and magnetic charges $q$ and $p$ is the Reissner-Nordström black hole

$$
d s^{2}=-f(\rho) d t^{2}+f^{-1}(\rho) d \rho^{2}+\rho^{2} d \Omega^{2}, \quad F=p \sin \theta d \theta \wedge d \phi+q \frac{d t \wedge d \rho}{\rho^{2}}
$$

where $d \Omega^{2}=d \theta^{2}+\sin ^{2} \theta d \phi^{2}$ is the metric on the two-sphere, and $f(\rho)$ is given in terms of the ADM mass $M$ and the charges $(p, q)$ by

$$
f(\rho)=1-\frac{2 G M}{\rho}+\frac{p^{2}+q^{2}}{\rho^{2}}
$$

For most of what follows, we set the Newton constant $G=1$. The Schwarzschild black hole is recovered in the neutral case $p=q=0$.

The solution (2.2) has a curvature singularity at $\rho=0$, with diverging curvature invariant $R_{\mu \nu} R^{\mu \nu} \sim 4\left(p^{2}+q^{2}\right)^{2} / \rho^{8}$. When $M^{2}<p^{2}+q^{2}$, this is a naked singularity and 

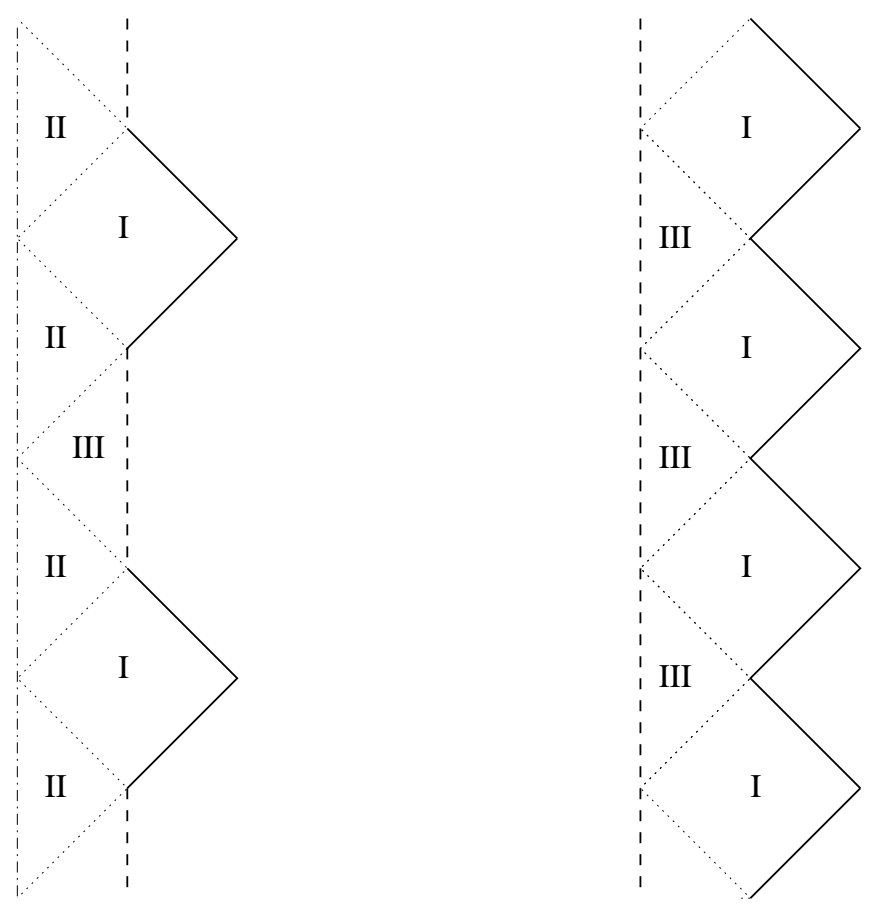

Figure 1: Penrose diagram of the non-extremal (left) and extremal (right) ReissnerNordström black holes. Dotted lines denote event horizons, dashed lines represent time-like singularities. The diagram on the left should be doubled along the dashed-dotted line.

the solution must be deemed unphysical. When $M^{2}>p^{2}+q^{2}$ however, there are two horizons at the zeros of $f(\rho)$,

$$
\rho_{ \pm}=M+\sqrt{M^{2}-p^{2}-q^{2}}
$$

which prevent the singularity to have any physical consequences for an observer at infinity, see the Penrose diagram on Figure 1. We shall denote by I, II, III the regions outside the horizon, between the two horizons and inside the inner horizon, respectively. Since the time-like component of the metric changes sign twice between regions I and III, the singularity at $\rho=0$ is time-like, and may be imputed to the existence of a physical source at $\rho=0$. This is unlike the Schwarzschild black hole, whose space-like singularity at $\rho=0$ raises more serious concerns.

Near the outer horizon, one may approximate

$$
f(\rho)=\frac{\left(\rho-\rho_{+}\right)\left(\rho-\rho_{-}\right)}{\rho^{2}} \sim \frac{\left(\rho_{+}-\rho_{-}\right)}{\rho_{+}^{2}} r
$$

where $\rho=\rho_{+}+r$, and the line element (2.2) by

$$
d s^{2} \sim\left[-\frac{\left(\rho_{+}-\rho_{-}\right)}{\rho_{+}^{2}} r d t^{2}+\frac{\rho_{+}^{2}}{\left(\rho_{+}-\rho_{-}\right)} \frac{d r^{2}}{r}\right]+\rho_{+}^{2} d \Omega_{2}^{2}
$$


Defining $t=2 \rho_{+}^{2} \tau /\left(\rho_{+}-\rho_{-}\right)$and $r=\eta^{2}$, the first term is recognized as Rindler space while the second term is a two-sphere of fixed radius,

$$
d s^{2}=\frac{4 \rho_{+}^{2}}{\rho_{+}-\rho_{-}}\left(-\eta^{2} d \tau^{2}+d \eta^{2}\right)+\rho_{+}^{2} d \Omega_{2}^{2} .
$$

Rindler space describes the patch of Minkowski space accessible to an observer $\mathcal{O}$ with constant acceleration $\kappa$. As spontaneous pair production takes place in the vacuum, $\mathcal{O}$ may observe only one member of that pair, while its correlated partner falls outside of $\mathcal{O}$ 's horizon. Hawking and Unruh have shown that, as a result, $\mathcal{O}$ detects a thermal spectrum of particles at temperature $T=\kappa /(2 \pi)$, where $\kappa$ is the acceleration, or "surface gravity" at the horizon [5, 6]. Equivalently, smoothness of the Wick-rotated geometry $\tau \rightarrow i \tau$ requires that $\tau$ be identified modulo $2 \pi i$. In terms of the inertial time $t$ at infinity, this requires $t \sim t+i \beta$ where $\beta$ is the inverse temperature

$$
\beta=\frac{1}{T}=\frac{4 \pi \rho_{+}^{2}}{\rho_{+}-\rho_{-}}
$$

Given an energy $M$ and a temperature $T$, it is natural to define the "BekensteinHawking" entropy $S_{B H}$ such that $d S_{B H} / d M=1 / T$ at fixed charges.

Exercice 1 By integrating (2.8), show that the entropy of a Reissner-Nordström black hole is equal to

$$
S_{B H}=\pi\left(M+\sqrt{M^{2}-p^{2}-q^{2}}\right)^{2}=\pi \rho_{+}^{2}
$$

Remarkably, the result is, up to a factor $1 /(4 G)$, just equal to the area of the horizon:

$$
S_{B H}=\frac{A}{4 G}
$$

This is a manifestation the following general statements, known as the "laws of black hole thermodynamics" (see e.g. [4, 7] and references therein):

0) The temperature $T=\kappa /(2 \pi)$ is uniform on the horizon;

I) Under quasi-static changes, $d M=(T / 4 G) d A+\phi d q+\chi d p$;

II) The horizon area always increases with time.

These statements rely purely on an analysis of the classical solutions to the action (2.1), and their singularities. The modifications needed to preserve the validity of these laws in the presence of corrections to the action (2.1) will be discussed in Section 6.2. 
The analogy of 0),I),II) with the usual laws of thermodynamics strongly suggests that it should be possible to identify the Bekenstein-Hawking entropy with the logarithm of the number of micro-states which lead to the same macroscopic black hole,

$$
S_{B H}=\log \Omega(M, p, q)
$$

where we set the Boltzmann constant to 1. In writing this equation, we took advantage of the "no hair" theorem which asserts that the black hole geometry, after transients, is completely specified by the charges measured at infinity.

Making sense of (2.11) microscopically requires quantizing gravity, which for us means using string theory. As yet, progress on this issue has mostly been restricted the case of extremal (or near-extremal) black holes, to which we turn now.

\subsection{Extremal Reissner-Nordström Black Holes}

In the discussion below (2.3), we left out one case, namely $M^{2}=p^{2}+q^{2}$. When this happens, the inner and outer horizons coalesce in a single degenerate horizon at $\rho=\sqrt{p^{2}+q^{2}}$, where the scale factor vanishes quadratically:

$$
f(\rho)=\left(1-\frac{\sqrt{p^{2}+q^{2}}}{\rho}\right)^{2} \sim \frac{r^{2}}{p^{2}+q^{2}}
$$

Such black holes are called "extremal", for reasons that will become clear below. In this case, defining $r=\left(p^{2}+q^{2}\right) / z$, we can rewrite the near-horizon geometry as

$$
d s \sim\left(p^{2}+q^{2}\right)\left[\frac{-d t^{2}+d z^{2}}{z^{2}}+d \Omega^{2}\right]
$$

which is now recognized as the product of two-dimensional Anti-de Sitter space $A d S_{2}$ times a two sphere. In contrast to (2.6), this is now a bona-fide solution of (2.1). The appearance of the $A d S_{2}$ factor raises the hope that such "extremal" black holes have an holographic description, although holography in $A d S_{2}$ is far less understood than in higher dimensions (see [8] for an early discussion).

An important consequence of $f(\rho)$ vanishing quadratically is that the Hawking temperature (2.8) is zero, so that the black hole no longer radiates: this is as it should, since otherwise its mass would go below the bound

$$
M^{2} \geq p^{2}+q^{2}
$$

producing a naked singularity. Black holes saturating this bound can be viewed as the stable endpoint of Hawking evaporation ${ }^{1}$, assuming that all charged particles are

\footnotetext{
${ }^{1}$ The evaporation end-point of neutral black holes is far less understood, and in particular leads to the celebrated "information paradox".
} 
massive. Moreover, the Bekenstein entropy remains finite

$$
S_{B H}=\pi\left(p^{2}+q^{2}\right)
$$

and becomes large in the limit of large charge. This is not unlike the large degeneracy of the lowest Landau level in condensed matter physics.

Upon embedding Einstein-Maxwell theory in extended supergravity, the "BPS" bound (2.14) is in fact a consequence of unitarity in a sector with non-vanishing central

charge $Z=\sqrt{p^{2}+q^{2}}$, see (3.17) below. The saturation of the bound implies that the black hole preserves some fraction of the supersymmetry of the vacuum. Since the corresponding representation of the supersymmetry algebra has smaller dimension that the generic one, such states are absolutely stable (unless they can pair up with an other extremal state with the same energy) [9]. They can be followed as the coupling is varied, which is part of the reason for their successful description in string theory. One important complication is that the extra massless scalar fields (or moduli) appearing in the supergravity Lagrangian, are sourced by the gauge fields, so have a non-trivial radial profile. Fortunately, in the case of extremal black holes, the near-horizon geometry, and in particular the horizon area, depends only on the electric-magnetic charges, being independent of the asymptotic values of the moduli (although it no longer takes the simple quadratic form (2.15), at tree-level it is still an homogeneous function of degree 2 in the charges). This is a consequence of the "attractor mechanism", which we will discuss at length in Sections 3 and 7.

As a side comment, it should be pointed out that even in supersymmetric theories, extremal black holes can exist which break all supersymmetries. In this case, the electromagnetic charges differ from the central charge, and the extremality bound is subject to quantum corrections. In this case, there may exist non-perturbative decay processes whereby an extremal black hole may break into smaller ones. The subject of non-supersymmetric extremal black holes has become of much interest recently, see e.g. $[10,11,12,13,14]$.

Exercice 2 Show that if black hole of mass and charge $(M, Q)$ breaks up into two black holes of mass and charge $\left(M_{1}, Q_{1}\right)$ and $\left(M_{2}, Q_{2}\right)$, then at least one of $M_{1} / Q_{1}$ and $M_{2} / Q_{2}$ must be smaller than $M / Q$. Conclude that quantum corrections should decrease the ratio $M / Q[14,15]$.

\subsection{Black Hole Counting via D-branes}

The ability of string theory to account microscopically for the Bekenstein-Hawking entropy of BPS black holes (2.15) is one of its most concrete successes. Since this 
subject is well covered in many reviews, we will only outline the argument, referring e.g. to $[16,17,18,19]$ for more details.

The main strategy, pioneered by Strominger and Vafa [20], is to represent the black hole as a bound state of solitons in string theory, and vary the coupling so that the degrees of freedom of these solitons become weakly coupled. The BPS property ensures that the number of micro-states will be conserved under this operation.

Consider for exemple 1/8 BPS black holes in Type II string theory on $T^{6}$, or $1 / 4$ BPS black holes on $K 3 \times T^{2}$ [21]. Both cases can treated simultaneously by writing the compact 6-manifold as $X=Y \times S_{1} \times S_{1}^{\prime}$, where $Y=T^{4}$ or $K 3$. Now consider a configuration of $Q_{6}$ D6-branes wrapped on $X, Q_{2}$ D2-branes wrapped on $S_{1} \times S_{1}^{\prime}$, $Q_{5}$ NS5-branes wrapped on $Y \times S_{1}$, carrying $N$ units of momentum along $S_{1}$. The resulting configuration is localized in the four non-compact directions, hence should be represented as a black hole of $\mathcal{N}=8$ or $\mathcal{N}=4$ supergravity $^{2}$. Its macroscopic entropy can be computed by studying the flow of the moduli with the above choice of charges, leading in either case to (Eq. (3.71) below)

$$
S_{B H}=2 \pi \sqrt{Q_{2} Q_{5} Q_{6} N}
$$

The micro-states correspond to open strings attached to the D2 and D6 branes, in the background of the NS5-branes. In the limit where $Y \times S_{1}^{\prime}$ is very small, they may be described by a two-dimensional field theory extending along the time and $S_{1}$ direction. The extremal micro-states correspond to the ground states of that field theory, with $N$ units of right-moving momentum along $S_{1}$. In the absence of the NS5-branes, the open strings are described at low energy by $U\left(Q_{2}\right) \times U\left(Q_{6}\right)$ gauge bosons together with bifundamental matter, which is known to flow to a CFT with central charge $c=6 Q_{2} Q_{6}$ in the infrared (see [18] for a detailed analysis of this point). In the presence of the NS5branes, localized at $Q_{5}$ points along $S_{1}^{\prime}$, the D2-branes generally break at the points where they intersect the NS5-branes. This effectively leads to $Q_{5} Q_{2}$ independent D2branes, hence a CFT with central charge $c_{\text {eff }}=6 Q_{2} Q_{5} Q_{6}$. By the Ramanujan-Hardy formula (Eq. (6.18) below), also known as the Cardy formula in the physics literature, the number of states carrying $N$ units of momentum grows exponentially as

$$
\Omega\left(Q_{2}, Q_{5}, Q_{6}, N\right) \sim \exp \left[2 \pi \sqrt{\frac{c_{\text {eff }}}{6} N}\right] \sim \exp \left[2 \pi \sqrt{Q_{2} Q_{5} Q_{6} N}\right]
$$

in precise agreement with the macroscopic answer (2.16).

\footnotetext{
${ }^{2}$ As usual in AdS/CFT correspondence, the closed string description is valid at large value of the t'Hooft coupling $g_{s} Q$, where $Q$ is any of the D-brane charges.
} 
While quantitatively successful, this argument has some obvious shortcomings. The degrees of freedom of the NS5-branes have been totally neglected, and the D2brane stretching between each of the NS5-branes were treated independently. A somewhat more tractable configuration can be obtained by T-dualizing along $S_{1}^{\prime}$, leading to a bound states of D1-D5 branes in the gravitational background of Kaluza-Klein monopoles [22]. The latter are purely gravitational solutions with orbifold singularities, so in principle can be treated by worldsheet techniques.

Key to this reasoning was the ability to lift the 4-dimensional black hole to a 5dimensional string, whose ground-state dynamics can be described by a two-dimensional "black string CFT", such that Cardy's formula is applicable. This indicates how to generalize the above argument to $1 / 2$-BPS black holes in $\mathcal{N}=2$ supergravity: any configuration of D0,D4 branes with vanishing D6-brane charge in type IIA string theory compactified on a Calabi-Yau threefold $X$ can be lifted in M-theory to a single M5brane wrapped around a general divisor (i.e. complex codimension one submanifold) $P$, with $N$ (the D0-brane charge) units of momentum along the M-theory direction [23]. The reduction of the $(0,2)$ tensor multiplet on the M5-brane worldvolume along the divisor $P$ leads to a $(0,4)$ SCFT in $1+1$ dimensions, whose left-moving central charge can be computed with some technical assumptions on $P$ :

$$
c_{L}=6 C(P)+c_{2} \cdot P
$$

Here, $C(P)$ is the self-intersection of $P$, while $c_{2}$ is the second Chern class of $X$. Using again Cardy's formula, this leads to

$$
\Omega \sim \exp \left[2 \pi \sqrt{\left.N\left[C(P)+\frac{1}{6} c_{2} \cdot P\right)\right]}\right]
$$

To leading order, this reproduces the macroscopic computation in $\mathcal{N}=2$ supergravity

$$
S_{B H}=2 \pi \sqrt{Q_{0} C\left(Q_{4}\right)}
$$

We shall return to formula (2.19) in Section 6 (Exercice 16), and show that the subleading contribution proportional to $c_{2}$ agrees with the macroscopic computation, provided one incorporates higher-derivative $R^{2}$ corrections.

\subsection{Counting $\mathcal{N}=4$ Dyons via Automorphic Forms}

In this subsection, we shall recall an interesting conjecture, due to E. Verlinde, H. Verlinde and R. Dijkgraaf (DVV), which purportedly relates the exact degeneracies of $1 / 4$-BPS states in $\mathcal{N}=4$ string theory, to Fourier coefficients of a certain automorphic form [24]. This conjecture has been the subject of much recent work, to which we will 
not be able to pay justice in this review. However, it plays an important inspirational role for some other conjectures relating black hole degeneracies and automorphic forms, that we will develop in Section 7.

Consider the heterotic string compactified on $T^{6}$, or equivalently the type II string on $K 3 \times T^{2}$. The moduli space factorizes into

$$
\frac{S l(2, \mathbb{R})}{U(1)} \times \frac{S O\left(6, n_{v}, \mathbb{R}\right)}{S O(6) \times S O\left(n_{v}\right)}
$$

with $n_{v}=22$. The first factor is the complex scalar in the $\mathcal{N}=4$ gravitational multiplet, and corresponds to the heterotic axio-dilaton $S$, or equivalently to the complexified Kähler modulus of $T^{2}$ on the type II side. Points in (2.21) related by an action of the duality group $\Gamma=S l(2, \mathbb{Z}) \times S O(6,22, \mathbb{Z})$ are conjectured to be equivalent under non-perturbative dualities.

The Bekenstein-Hawking entropy for 1/4-BPS black holes is given by [25]

$$
S_{B H}=\pi \sqrt{\left(\vec{q}_{e} \cdot \vec{q}_{e}\right)\left(\vec{q}_{m} \cdot \vec{q}_{m}\right)-\left(\vec{q}_{e} \cdot \vec{q}_{m}\right)^{2}}
$$

where $\vec{q}_{e}$ and $\vec{q}_{m}$ are the electric and magnetic charges in the natural heterotic polarization. $\left(\vec{q}_{m}, \vec{q}_{e}\right)$ transform as a doublet of $S O\left(6, n_{v}\right)$ vectors under $S l(2)$. Equation (2.22) is manifestly invariant under the continuous group $S l(2, \mathbb{R}) \times S O(6,22, \mathbb{R})$, a fortiori under its discrete subgroup $\Gamma$.

DVV proposed that the exact degeneracies should be given by the Fourier coefficients of the inverse of $\Phi_{10}$, the unique cusp form of $S p(4, \mathbb{Z})$ with modular weight 10:

$$
\Omega\left(\vec{q}_{e}, \vec{q}_{m}\right) \stackrel{?}{=} \int_{\gamma} d \tau \frac{1}{\Phi_{10}(\tau)} e^{-i\left(\rho \vec{q}_{m}^{2}+\sigma \vec{q}_{e}^{2}+2 \nu \vec{q}_{e} \cdot \vec{q}_{m}\right)}
$$

Here, $\tau=\left(\begin{array}{ll}\rho & \nu \\ \nu & \sigma\end{array}\right)$ parameterizes Siegel's upper half plane $S p(4, \mathbb{R}) / U(2)$ and $\gamma$ is the contour $0 \leq \rho, \nu, \sigma \leq 2 \pi$. One may think of $\omega$ as the period matrix of an auxiliary genus 2 Riemann surface, with modular group $S p(4, \mathbb{Z})$. The cusp form $\Phi_{10}$ has an infinite product representation

$$
\Phi_{10}(\tau)=e^{i(\rho+\sigma+\nu)} \prod_{(k, l, m)>0}\left(1-e^{i(k \rho+l \sigma+m)}\right)^{c\left(4 k l-m^{2}\right)}
$$

where $c(k)$ are the Fourier coefficients of the elliptic genus of $K 3$,

$$
\chi_{K 3}(\rho, \nu)=\sum_{h \geq 0, m \in \mathbb{Z}} c\left(4 h-m^{2}\right) e^{2 \pi i(h \rho+m z)}=24\left(\frac{\theta_{3}(\rho, z)}{\theta_{3}(\rho)}\right)^{2}-2 \frac{\left(\theta_{4}^{4}(\rho)-\theta_{2}^{4}(\rho)\right) \theta_{1}^{2}(\rho, z)}{\eta^{6}(\rho)}
$$


which shows that the Fourier coefficients obtained in this fashion are (in general nonpositive) integers.

The r.h.s. of (2.23) is manifestly invariant under continuous rotations in $S O(6,22, \mathbb{R})$, hence under its discrete subgroup $S O(6,22, \mathbb{Z})$. The invariance under $S l(2, \mathbb{Z})$ is more subtle, and uses the embedding of $S l(2, \mathbb{Z})$ inside $S p(4, \mathbb{Z})$; using the modular invariance of $\Phi_{10}$, one can cancel the action of $S l(2, \mathbb{Z})$ by a change of contour $\gamma \rightarrow \gamma^{\prime}$, and deform $\gamma^{\prime}$ back to $\gamma$ while avoiding singularities.

As a consistency check on this conjecture, one can extract the large charge behavior of $\Omega\left(\vec{q}_{e}, \vec{q}_{m}\right)$ by computing the contour integral in (2.23) by residues, and obtain agreement with (2.22) [24].

Exercice 3 By picking the residue at the divisor $D=\rho \sigma+\nu-\nu^{2} \sim 0$ and using $\Phi_{10}=D^{2} \eta^{24}\left(\rho^{\prime}\right) \eta^{24}\left(\sigma^{\prime}\right) / \operatorname{det}^{12}(\tau)$ where $\rho^{\prime}=-\frac{\sigma}{\rho \sigma-\nu^{2}}, \sigma^{\prime}=-\frac{\rho}{\rho \sigma-\nu^{2}}$, reproduce the leading charge behavior (2.22). You may seek help from [24, 26].

A recent "proof" of the DVV conjecture has recently been given by lifting 4D black holes with unit D6-brane charge to 5D, and using the Strominger-Vafa relation between degeneracies of 5D black hole and the elliptic genus of $H i l b(K 3)$ [27]. We will return to this $4 \mathrm{D} / 5 \mathrm{D}$ lift in Section 3.5. The conjecture has also been generalized to other $\mathcal{N}=4$ "CHL" models with different values of $n_{v}$ in $(2.21)$ [28, 29, 30]. More recently, the $S p(4, \mathbb{Z})$ symmetry has been motivated by representing $1 / 4$-BPS dyons as string networks on $T^{2}$, which lift to M2-branes with genus 2 topology [31]. Despite this interpretation, it is fair to say that the origin of $S p(4)$ remains rather mysterious. In Section 7, we will formulate a similar conjecture, which relies on the 3-dimensional U-duality group $S O(8,24, \mathbb{Z})$ obtained by reduction on a thermal circle.

\section{Special Geometry And Black Hole Attractors}

In this section, we expose the formalism of special geometry, which governs the couplings of vector multiplets in $\mathcal{N}=2, D=4$ supergravity. We then derive the attractor flow equations, governing the radial evolution of the scalars in spherically BPS geometries. Finally, we illustrate these these constructions in the context of "very special" supergravity theories, which are simple toy models of $\mathcal{N}=2$ supergravity with symmetric moduli spaces. We follow the notations of [32], which gives a good overview of the essentials of special geometry. Useful reviews of the attractor mechanism include $[33,34,35]$. 


\section{$3.1 \mathcal{N}=2$ SUGRA and Special Geometry}

A general "ungauged" $\mathcal{N}=2$ supergravity theory in 4 dimensions may be obtained by combining massless supersymmetric multiplets with spin less or equal to 2 :

i) The gravity multiplet, containing the graviton $g_{\mu \nu}$, two gravitini $\psi_{\mu}^{\alpha}$ and one Abelian gauge field known as the graviphoton;

ii) $n_{V}$ vector multiplets, each consisting of one Abelian gauge field $A_{\mu}$, two gaugini $\lambda^{\alpha}$ and one complex scalar. The complex scalars $z_{i}$ take values in a projective special Kähler manifold $\mathcal{M}_{V}$ of real dimension $2 n_{V}$.

iii) $n_{H}$ hypermultiplets, each consisting of two complex scalars and two hyperinis $\psi, \tilde{\psi}$. The scalars take values in a quaternionic-Kähler space $\mathcal{M}_{\mathcal{H}}$ of real dimension $4 n_{H}$.

Tensor multiplets are also possible, and can be dualized into hypermultiplets with special isometries. At two-derivative order, vector multiplets and hypermultiplets interact only gravitationally ${ }^{3}$. We will concentrate on the gravitational and vector multiplet sectors, which control the physics of charged BPS black holes. Nevertheless, we will encounter hypermultiplet moduli spaces in Section 7.2.5, when reducing the solutions to three dimensions.

The couplings of the vector multiplets, including the geometry of the scalar manifold $\mathcal{M}_{V}$, are conveniently described by means of a $S p\left(2 n_{V}+2\right)$ principal bundle $\mathcal{E}$ over $\mathcal{M}_{V}$, and its associated bundle in the fundamental representation of $S p\left(2 n_{V}+2\right)$. The origin of the symplectic symmetry lies in electric-magnetic duality, which mixes the $n_{V}$ vectors $\mathcal{A}_{\mu}$ and the graviphoton $\mathcal{A}_{\mu}$ together with their magnetic duals. Denoting a section $\Omega$ by its coordinates $\left(X^{I}, F_{I}\right)$, the antisymmetric product

$$
\left\langle\Omega, \Omega^{\prime}\right\rangle=\left\langle X^{I} F_{I}^{\prime}-X^{\prime I} F_{I}\right\rangle
$$

endows the fibers with a phase space structure, derived from the symplectic form $\langle d \Omega, d \Omega\rangle=d X^{I} \wedge d F_{I}$.

The geometry of the scalar manifold $\mathcal{M}_{V}$ is completely determined by a choice of a holomorphic section $\Omega(z)=\left(X^{I}(z), F_{I}(z)\right)$ taking value in a Lagrangian cone, namely a dilation invariant subspace such that $d X^{I} \wedge d F_{I}=0$. At generic points, one may express $F_{I}$ in terms of their canonical conjugate $X^{I}$ via a characteristic function $F\left(X^{I}\right)$ known as the prepotential:

$$
F_{I}=\frac{\partial F}{\partial X^{I}}, \quad F\left(X^{I}\right)=\frac{1}{2} X^{I} F_{I} .
$$

\footnotetext{
${ }^{3}$ This is no longer true in "gauged" supergravities, where some of the hypermultiplets become charged under the vectors.
} 
The second relation reflects the homogeneity of the Lagrangian, and implies that $F$ is an homogeneous function of degree 2 in the $X^{I}$. At generic points, the sections $X^{I}\left(I=0 \ldots n_{V}\right)$ may be chosen as projective holomorphic coordinates on $\mathcal{M}_{V}$ equivalently, the $n_{V}$ ratios $z^{i}=X^{i} / X^{0}\left(i=1 \ldots n_{V}\right)$ may be taken as the holomorphic coordinates; these are known as (projective) special coordinates. Note however that a choice of $F$ breaks manifest symplectic invariance, so special coordinates may not always be the most convenient ones.

Exercice 4 Show that a symplectic transformation $\left(X^{I}, F_{I}\right) \rightarrow\left(F_{I},-X^{I}\right)$, turns the prepotential into its Legendre transform.

Once the holomorphic section $\Omega(z)$ is given, the metric on $\mathcal{M}_{V}$ may be obtained from the Kähler potential

$$
K\left(z^{i}, \bar{z}^{i}\right)=-\log [i\langle\bar{\Omega}, \Omega\rangle]=-\log \left[i\left(\bar{X}^{I} F_{I}-X^{I} \bar{F}_{I}\right)\right]
$$

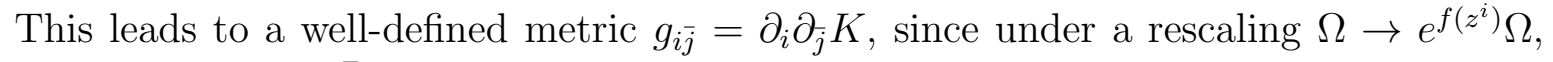
$K \rightarrow K-f(z)-\bar{f}(\bar{z})$ changes by a Kähler transformation. Equivalently, $\Omega$ should be viewed as a section of $\mathcal{E} \otimes \mathcal{L}$ where $\mathcal{L}$ is the Hodge bundle over $\mathcal{M}_{V}$, namely a line bundle whose curvature is equal to the Kähler form; its connection one-form is just $\left(\partial_{i} K d z^{i}-\partial_{\bar{i}} K d z^{\bar{i}}\right) /(2 i)$. The rescaled section $\tilde{\Omega}=e^{K / 2} \Omega$ is then normalized to 1 , and transforms by a phase under holomorphic rescalings of $\Omega$. For later purposes, it will be convenient to introduce the derived section $U_{i}=D_{i} \tilde{\Omega}=\left(f_{i}^{I}, h_{i I}\right)$ where

$$
\begin{aligned}
f_{i}^{I} & =e^{K / 2} D_{i} X^{I}=e^{K / 2}\left(\partial_{i} X^{I}+\partial_{i} K X^{I}\right) \\
h_{i I} & =e^{K / 2} D_{i} F_{I}=e^{K / 2}\left(\partial_{i} F_{I}+\partial_{i} K F_{I}\right)
\end{aligned}
$$

The metric may thus be reexpressed as

$$
g_{i \bar{j}}=-i\left\langle U_{i}, \bar{U}_{\bar{j}}\right\rangle=i\left(f_{i}^{I} \bar{h}_{\bar{j} I}-h_{i I} \bar{f}_{\bar{j}}^{I}\right)
$$

After some algebra, one may show that the Riemann tensor on $\mathcal{M}_{V}$ takes the form

$$
R_{i \bar{j} k \bar{l}}=g_{i \bar{j}} g_{k \bar{l}}+g_{i \bar{l}} g_{k \bar{j}}-e^{2 K} C_{i k m} \bar{C}_{\bar{j} \bar{l} \bar{n}} g^{m \bar{n}}
$$

where $C_{i j k}$ is a holomorphic, totally symmetric tensor ${ }^{4}$

$$
C_{i j k}=e^{-K}\left\langle D_{i} U_{j}, U_{k}\right\rangle
$$

\footnotetext{
${ }^{4}$ We follow the standard notation in the topological string literature, which differs from [32] by a factor of $e^{K}$.
} 
The foregoing formalism was in fact geared to produce a solution of Equation (3.7), which may be taken as the definition of a projective special Kähler manifold.

The kinetic terms of the $n_{V}+1$ Abelian gauge fields (including the graviphoton) may also be obtained from the holomorphic section $\Omega$ as

$$
\begin{aligned}
\mathcal{L}_{\text {Maxwell }} & =\int-\operatorname{Im} \mathcal{N}_{I J} \mathcal{F}^{I} \wedge \star \mathcal{F}^{J}+\operatorname{Re} \mathcal{N}_{I J} \mathcal{F}^{I} \wedge \mathcal{F}^{J} \\
& =\int \operatorname{Im}\left[\overline{\mathcal{N}}_{I J} \mathcal{F}^{I-} \wedge \star \mathcal{F}^{J-}\right]+\text { total der. }
\end{aligned}
$$

where $\mathcal{F}^{I-}=\left(\mathcal{F}^{I}-i \star \mathcal{F}^{I}\right) / 2, I=0 \ldots n_{V}$ is the anti-self dual part of the field-strength, and $\mathcal{N}_{I J}$ is defined by the relations

$$
F_{I}=\mathcal{N}_{I J} X^{J}, \quad h_{i I}=\overline{\mathcal{N}}_{I J} f_{i}^{J}
$$

In term of the prepotential $F$ and its Hessian $\tau_{I J}=\partial_{I} \partial_{J} F$,

$$
\mathcal{N}_{I J}=\bar{\tau}_{I J}+2 i \frac{(\operatorname{Im} \tau \cdot X)_{I}(\operatorname{Im} \tau \cdot X)_{J}}{X \cdot \operatorname{Im} \tau \cdot X}
$$

While $\operatorname{Im} \tau_{I J}$ has indefinite signature $\left(1, n_{V}\right), \operatorname{Im} \mathcal{N}_{I J}$ is a negative definite matrix, as appropriate for the positive definiteness of the gauge kinetic terms in (3.9).

Exercice 5 For later use, prove the relations

$$
K=-\log \left[-2 X^{I}[\operatorname{Im} \mathcal{N}]_{I J} \bar{X}^{J}\right], \quad f_{i}^{I}[\operatorname{Im} \mathcal{N}]_{I J} X^{J}=0
$$

In order to study the invariance of (3.9) under electric-magnetic duality, it is useful to introduce the dual vector

$$
\mathcal{G}_{I ; \mu \nu}=\frac{1}{2} \frac{\partial \mathcal{L}_{\text {Maxwell }}}{\partial \mathcal{F}^{I ; \mu \nu}}=\operatorname{Re} \mathcal{N}_{I J} \mathcal{F}^{J}+\operatorname{Im} \mathcal{N}_{I J} \star \mathcal{F}^{I}
$$

Under symplectic transformations, $\mathcal{N}$ transforms as a "period matrix" $\mathcal{N} \rightarrow(C+$ $D \mathcal{N})(A+B \mathcal{N})^{-1}$, while the field strengths $\left(\mathcal{F}^{I-}, \mathcal{G}_{I}^{-}=\overline{\mathcal{N}}_{I J} \mathcal{F}_{\mu \nu}^{J-}\right)$ transform as a symplectic vector, leaving (3.9) invariant. The electric and magnetic charges $\left(p^{I}, q_{I}\right)$ are measured by the integral on a 2 -sphere at spatial infinity of $\left(\mathcal{F}^{I-}, \mathcal{G}_{I}^{-}\right)$, and transform as a symplectic vector too.

One linear combination of the $n_{V}+1$ field-strengths, the graviphoton

$$
T_{\mu \nu}^{-}=-2 i e^{K / 2} X^{I} \operatorname{Im} \mathcal{N}_{I J} \mathcal{F}_{\mu \nu}^{J-}=e^{K / 2}\left(X^{I} \mathcal{G}_{I}^{-}-F_{I} \mathcal{F}^{I-}\right)
$$

plays a distinguished rôle, as its associated charge measured at infinity

$$
Z=e^{K / 2}\left(q_{I} X^{I}-p^{I} F_{I}\right):=e^{K / 2} W(X)
$$


appears as the central charge in $\mathcal{N}=2$ supersymmetry algebra,

$$
\left\{Q_{\alpha}^{i}, \bar{Q}_{\dot{\alpha} j}\right\}=P_{\mu} \sigma_{\alpha \dot{\alpha}}^{\mu} \delta_{j}^{i}, \quad\left\{Q_{\alpha}^{i}, Q_{\beta}^{j}\right\}=Z \epsilon^{i j} \epsilon_{\alpha \beta}
$$

In particular, there is a Bogomolony-Prasad-Sommerfeld (BPS) bound on the mass

$$
M^{2} \geq|Z|^{2} m_{P}^{2}
$$

where $m_{P}$ is the (duality invariant) 4-dimensional Planck scale, which is saturated when the state preserves 4 supersymmetries out of the 8 supersymmetries of the vacuum.

\section{$3.2 \mathcal{N}=2$ SUGRA and String Theory}

There are several ways to obtain $\mathcal{N}=2$ supergravities in 4 dimensions from string theory. Type IIB string compactified on a Calabi-Yau three-fold $Y$ leads to a $\mathcal{N}=2$ supergravity with $n_{V}=h_{2,1}(Y)$ vector multiplets and $n_{H}=h_{1,1}(Y)+1$ hypermultiplets. The scalars in $\mathcal{M}_{V}$ parameterize the complex structure of the Calabi-Yau metric on $Y$. The associated vector fields are the reduction of the 10D Ramond-Ramond 4-form on the various 3 -cycles in $H_{3}(Y, \mathbb{R})$. The holomorphic section is then given by the periods

of the holomorphic 3 -form $\Omega$ (abusing the notation) on a symplectic basis $\left(A^{I}, B_{I}\right)$ of $H_{3}(Y, \mathbb{R})$ :

$$
X^{I}=\int_{A^{I}} \Omega, \quad F_{I}=\int_{B^{I}} \Omega
$$

The Kähler potential on the moduli of complex structures is just

$$
K=-\log \left[i \int_{Y} \Omega \wedge \bar{\Omega}\right]
$$

which agrees with (3.3) by Riemann's bilinear identity. As we shall see later, it is determined purely at tree-level, and can be computed purely in field theory. The central charge of a state with electric-magnetic charges $p^{I}, q_{I}$ may be rewritten as

$$
Z=\frac{\int_{\gamma} \Omega}{\sqrt{i \int_{Y} \Omega \wedge \bar{\Omega}}}
$$

where $\gamma=q_{I} A^{I}-p^{I} B_{I}$, and is recognized as the mass of a D3-brane wrapped on a special Lagrangian 3-cycle $\gamma \in H_{3}(Y, \mathbb{Z})$.

On the other hand, the scalars in $\mathcal{M}_{H}$ parameterize the complexified Kähler structure of $Y$, the fluxes (or more appropriately, Wilson lines) of the Ramond-Ramond two-forms along $H_{\text {even }}(Y, \mathbb{R})$, as well as the axio-dilaton. The axio-dilaton, zero and sixform RR potentials form a "universal hypermultiplet" sector inside $\mathcal{M}_{H}$. In contrast 
to the vector-multiplet metric, the hyper-multiplet metric receives one-loop and nonperturbative corrections from Euclidean D-branes and NS-branes wrapped on $H_{\text {even }}(Y)$.

The situation in type IIA string compactified on a Calabi-Yau three-fold $\tilde{Y}$ is reversed: the vector-multiplet moduli space described the complexified Kähler structure of $\tilde{Y}$, while the hypermultiplet moduli space describes its complex structure, together with the Wilson lines of the Ramond-Ramond forms along $H_{\text {odd }}(\tilde{Y})$ and the axiodilaton. As in IIB, the vector-multiplet moduli space is determined at tree-level only, but receives $\alpha^{\prime}$ corrections. Letting $J=B_{N S}+i \omega_{K}$ be the complexified Kähler form, $\gamma^{A}$ be a basis of $H_{1,1}(\tilde{Y}, \mathbb{Z})$ and $\gamma_{A}$ the dual basis of $H_{2,2}(\tilde{Y}, \mathbb{Z})$, the holomorphic section $\Omega$ (not to be confused with the holomorphic three-form on $\tilde{Y}$ ) is determined projectively by the special coordinates

$$
X^{A} / X^{0}=\int_{\gamma^{A}} J, \quad F_{A} / X^{0}=\int_{\gamma_{A}} J \wedge J
$$

In the limit of large volume, the Kähler potential (in the gauge $X^{0}=1$ ) is given by the volume

$$
K=-\log \int_{\tilde{Y}} J \wedge J \wedge J
$$

originating from the cubic prepotential

$$
F=-\frac{1}{6} C_{A B C} \frac{X^{A} X^{B} X^{C}}{X^{0}}+\ldots
$$

Here, $C_{A B C}$ are the intersection numbers of the 4-cycles $\gamma_{A, B, C}$. At finite volume, there are corrections to (3.23) from worldsheet instantons wrapping effective curves in $H_{2}^{+}(\tilde{Y}, \mathbb{Z})$, to which we will return in Section 4.3. The central charge following from $(3.23)$ is

$$
Z=e^{K / 2} X^{0}\left(q_{0}+q_{A} \int_{\gamma^{A}} J-p^{A} \int_{\gamma_{A}} J \wedge J-p^{0} \int_{\tilde{Y}} J \wedge J \wedge J\right)
$$

so that $q_{0}, q_{A}, p^{A}, p^{0}$ can be identified as the D0,D2,D4 and D6 brane charge, respectively.

While (3.23) expresses the complete prepotential in terms of the geometry of $\tilde{Y}$, the most practical way of computing it is to use mirror symmetry, which relates type IIA compactified on $\tilde{Y}$ to type IIB compactified on $Y$, where $(Y, \tilde{Y})$ form a "mirror pair"; this implies in particular that $h_{1,1}(Y)=h_{2,1}(\tilde{Y})$ and $h_{1,1}(\tilde{Y})=h_{2,1}(Y)$ (see [36] for a review).

On the other hand, the tree-level metric on the hypermultiplet moduli space $\mathcal{M}_{H}$ in type IIA compactified on $\tilde{Y}$ may be obtained from the vector-multiplet metric $\mathcal{M}_{V}$ 
in type IIB compactified on the same Calabi-Yau $\tilde{Y}$, by compactifying on a circle $S^{1}$ to 3 dimensions, T-dualizing along $S^{1}$ and decompactifying back to 4 dimensions. We shall return to this "c-map" procedure in Section 7.2.5.

Finally, another way to obtain $\mathcal{N}=2$ supergravity in 4 dimensions is to compactify the heterotic string on $K 3 \times T^{2}$. Since the heterotic axio-dilaton is now a vectormultiplet, $\mathcal{M}_{V}$ now receives loop and instanton corrections, while $\mathcal{M}_{H}$ is determined purely at tree-level (albeit with $\alpha^{\prime}$ corrections).

\subsection{Attractor Flows and Bekenstein-Hawking Entropy}

We now turn to static, spherically symmetric BPS black hole solutions of $\mathcal{N}=2$ supergravity. The assumed isometries lead to the metric ansatz

$$
d s^{2}=-e^{2 U} d t^{2}+e^{-2 U}\left(d r^{2}+r^{2} d \Omega_{2}^{2}\right)
$$

where $d \Omega_{2}^{2}=d \theta^{2}+\sin ^{2} \theta d \phi^{2}$ is the round metric on $S^{2}$, and $U$ depends on $r$ only. We took advantage of the BPS property to restrict to flat 3D spatial slices ${ }^{5}$. Moreover, the scalars $z^{i}$ in the vector multiplet moduli space are taken to depend on $r$ only. The gauge fields are uniquely determined by the equations of motion and Bianchi identities:

$$
\mathcal{F}^{I-}=\frac{1}{2}\left[p^{I}-i[\operatorname{Im} \mathcal{N}]^{I J}\left(q_{J}-[\operatorname{Re} \mathcal{N}]_{J K} p^{K}\right)\right] \cdot\left[\sin \theta d \theta \wedge d \phi-i \frac{e^{2 U}}{r^{2}} d t \wedge d r\right]
$$

where $\left(p^{I}, q_{I}\right)$ are the magnetic and electric charges, and $[\operatorname{Im} \mathcal{N}]^{I J}=[\operatorname{Im} \mathcal{N}]_{I J}^{-1}$.

Assuming that the solution preserves half of the 8 supersymmetries, the gravitino and gaugino variations lead to a set of first-order equations $[38,39,40,34]^{6}$

$$
\begin{aligned}
& r^{2} \frac{d U}{d r}=-|Z| e^{U} \\
& r^{2} \frac{d z^{i}}{d r}=-2 e^{U} g^{i \bar{j}} \partial_{\bar{j}}|Z|
\end{aligned}
$$

where $Z$ is the central charge defined in (3.15). These equations govern the radial evolution of $U$ and $z^{i}(r)$, and are usually referred to as "attractor flow equations", for reasons which will become clear shortly. Defining $\mu=e^{-U}$, so that $r^{2} d \mu / d r=|Z|$, the second equation may be cast in the form of a gradient flow, or RG flow,

$$
\mu \frac{d z^{i}}{d \mu}=-g^{i \bar{j}} \partial_{\bar{j}} \log |Z|^{2}
$$

\footnotetext{
${ }^{5}$ This condition may be relaxed if one allows for a non-trivial profile of the hypermultiplets [37].

${ }^{6}$ We shall provide a full derivation of $(3.27),(3.28)$ in Section 7 , but for now we accept them and proceed with their consequences.
} 
The black hole horizon is reached when the component $g_{t t}$ of the metric goes to zero, i.e. when $\mu$ goes to infinity. The scalars $z^{i}$ settle to the minima of the potential $\log |Z|^{2}$, which depends only on the electric and magnetic charges; the vector multiplet scalars are therefore "attracted" to fixed values at the horizon $z_{*}^{i}$, independent of their asymptotic values at $r \rightarrow \infty$. This is in fact a consequence of extremality rather than supersymmetry, as was first noticed in [40].

We shall assume that the charges $p^{I}, q_{I}$ are chosen such that at the attractor point, $Z \neq 0$, since otherwise the solution becomes singular. Equation (3.27) may be easily integrated near the horizon,

$$
\mu=e^{-U} \sim\left|Z_{*}\right| / r
$$

Defining $z=\left|Z_{*}\right|^{2} / r$, it is easy to see that the near-horizon metric becomes $A d S_{2} \times S^{2}$, as in (2.13), where the prefactor $\left(p^{2}+q^{2}\right)$ is replaced by $\left|Z_{*}\right|^{2}$. The Bekenstein-Hawking entropy is one quarter of the horizon area,

$$
S_{B H}=\frac{1}{4} \cdot 4 \pi \lim _{r \rightarrow 0} e^{-2 U} r^{2}=\pi\left|Z_{*}\right|^{2}
$$

This is a function of the electric and magnetic charges only, by virtue of the attractor mechanism, except for possible discrete labels (or "area codes") corresponding to different basins of attraction.

We shall now put these results in a more manageable form, by making use of some special geometry identities discussed in Section 3. First, using the derived section $U_{i}=\left(f_{i}^{I}, h_{i I}\right)$ defined in (3.4) and the property (3.10), one easily finds

$$
\partial_{i} Z=f_{i}^{I}\left(q_{I}-\overline{\mathcal{N}}_{I J} p^{J}\right)-\frac{1}{2} Z \partial_{i} K, \quad \partial_{i} Z=\frac{1}{2} Z \partial_{i} K
$$

so that

$$
\frac{\partial_{i}|Z|}{|Z|}=\frac{1}{2}\left(\frac{\partial_{i} Z}{Z}+\frac{\partial_{i} \bar{Z}}{\bar{Z}}\right)=\frac{1}{Z} f_{i}^{I}\left(q_{I}-\overline{\mathcal{N}}_{I J} p^{J}\right)
$$

This allows to rewrite $(3.28)$ as

$$
r^{2} \frac{d z^{i}}{d r}=-\sqrt{\frac{Z}{\bar{Z}}} e^{U} g^{i \bar{j}} \bar{f}_{\bar{j}}^{J}\left(q_{I}-\mathcal{N}_{I J} p^{J}\right)
$$

The stationary value of $z^{i}$ at the horizon is thus obtained by setting the rhs of this equation to zero, i.e.

$$
f_{i}^{J}\left(q_{I}-\overline{\mathcal{N}}_{I J} p^{J}\right)=0
$$

The rectangular matrix $f_{i}^{I}$ has a unique zero eigenvector, given by the second equality in (3.12). Hence, (3.35) implies

$$
q_{I}-\overline{\mathcal{N}}_{I J} p^{J}=C \operatorname{Im} \mathcal{N}_{I J} X^{J}
$$


Contracting either side with $\bar{X}^{I}$ and using the first equation in (3.12) allows to compute the value of $\alpha$,

$$
C=-2 \bar{Z} e^{K / 2}
$$

Moreover, using again (3.10), one may rewrite (3.36) and its complex conjugate, equivalently as two real equations

$$
p^{I}=\operatorname{Im}\left(C X^{I}\right), \quad q_{I}=\operatorname{Im}\left(C F_{I}\right)
$$

Making use of the fact that near the horizon, $e^{-U} \sim\left|Z_{*}\right| / r$, it is convenient to rescale the holomorphic section $\Omega=\left(X^{I}, F_{I}\right)$ into

$$
\left(\begin{array}{c}
Y^{I} \\
G_{I}
\end{array}\right)=2 i r e^{K / 2-U} \sqrt{\frac{\bar{Z}}{Z}}\left(\begin{array}{c}
X^{I} \\
F_{I}
\end{array}\right)
$$

in such a way that

$$
e^{-K(Y, \bar{Y})}=4 r^{2} e^{-2 U}, \quad \arg W(Y)=\pi / 2
$$

where we defined, in line with (3.3) and (3.15),

$$
K(Y, \bar{Y})=-\log \left[i\left(\bar{Y}^{I} G_{I}-Y^{I} \bar{G}_{I}\right)\right], \quad W(Y)=q_{I} Y^{I}-p^{I} G_{I}
$$

In this fashion, we have incorporated the geometric variable $U$ into the symplectic section $\left(Y^{I}, G_{I}\right)$, and fixed the phase. In this new "gauge" ${ }^{7},(3.38)$ and (3.31) simplify into

$$
\begin{aligned}
\left(\begin{array}{c}
p^{I} \\
q_{I}
\end{array}\right) & =\operatorname{Re}\left(\begin{array}{c}
Y^{I} \\
G_{I}
\end{array}\right) \\
S_{B H} & =\frac{\pi}{4} e^{-K(Y, \bar{Y})}=\frac{i \pi}{4}\left[\bar{Y}^{I} G_{I}-Y^{I} \bar{G}_{I}\right]
\end{aligned}
$$

These equations, some times known as "stabilization equations", are the most convenient way of summarizing the endpoint of the attractor mechanism, as will become apparent in the next subsection.

\subsection{Bekenstein-Hawking entropy and Legendre transform}

A key observation for later developments is that the Bekenstein-Hawking entropy (3.42) is simply related by Legendre transform to the tree-level prepotential $F$. To see this,

\footnotetext{
${ }^{7}$ This is an abuse of language, since the scale factor is a priori not a holomorphic function of $z^{i}$.
} 
note that the first equation in (3.42) is trivially solved by setting $Y^{I}=p^{I}+i \phi^{I}$, where $\phi^{I}$ is real. The entropy is then rewritten as

$$
\begin{aligned}
S_{B H} & =\frac{i \pi}{4}\left[\left(Y^{I}-2 i \phi^{I}\right) G_{I}-\left(\bar{Y}^{I}+2 i \phi^{I}\right) \bar{G}_{I}\right] \\
& =\frac{i \pi}{2}[F(Y)-\bar{F}(\bar{Y})]+\frac{\pi}{2} \phi^{I}\left[G_{I}+\bar{G}_{I}\right]
\end{aligned}
$$

where, in going from the second to the third line, we used the homogeneity of the prepotential, $Y^{I} G_{I}=2 F(Y)$. On the other hand, the second stabilization equation yields

$$
q_{I}=\frac{1}{2}\left(G_{I}+\bar{G}_{I}\right)=\frac{1}{2 i}\left(\frac{\partial F}{\partial \phi^{I}}-\frac{\partial \bar{F}}{\partial \phi^{I}}\right)
$$

Thus, defining

$$
\mathcal{F}\left(p^{I}, \phi^{I}\right)=-\pi \operatorname{Im}\left[F\left(p^{I}+i \phi^{I}\right)\right]
$$

the last equation in (3.45) becomes

$$
S_{B H}\left(p^{I}, q_{I}\right)=\left\langle\mathcal{F}\left(p^{I}, \phi^{I}\right)+\pi \phi^{I} q_{I}\right\rangle_{\phi^{I}}
$$

where the r.h.s. is evaluated at its extremal value with respect to $\phi^{I}$. In usual thermodynamical terms, this implies that $\mathcal{F}\left(p^{I}, \phi^{I}\right)$ should be viewed as the free energy of an ensemble of black holes in which the magnetic charge $p^{I}$ is fixed, but the electric charge $q_{I}$ is free to fluctuate at an electric potential $\pi \phi^{I}$. The implications of this simple observation will be profound in Section 5.3, when we discuss the higher-derivative corrections to the Bekenstein-Hawking entropy.

Exercice 6 Apply this formalism to show that the entropy of a D0-D4 bound state in type IIA string theory compactified on a Calabi-Yau three-fold, in the large charge regime, is given by

$$
S_{B H}=2 \pi \sqrt{-C_{A B C} p^{A} p^{B} p^{C} q_{0}}
$$

and compare to $(2.20)$.

Exercice 7 Show that the Bekenstein-Hawking entropy (3.43) can be obtained by extremizing

$$
\Sigma(Y, \bar{Y})=\frac{\pi}{4} e^{-K(Y, \bar{Y})}+2 i[W(Y)-\bar{W}(Y)]
$$

with respect to $Y, \bar{Y}$, where $K(Y, \bar{Y})$ and $W(Y)$ are defined in $(3.41)$ [41, 42]. Note that (3.48) is recovered by extremizing over $\operatorname{Re}(Y)$. 


\subsection{Very Special Supergravities and Jordan Algebras}

In the remainder of this section, we illustrate the previous results on a special class of $\mathcal{N}=2$ supergravities, whose vector-multiplet moduli spaces are given by symmetric spaces. These are interesting toy models, which arise in various truncations of string compactifications. Moreover, they are related to by analytic continuation to $\mathcal{N}>2$ theories, which will be further discussed in Section 7.

The simplest way to construct these models is to start from 5 dimensions [43]: the vector multiplets consist of one real scalar for each vector, and their couplings are given by

$$
S=\int d^{5} x \sqrt{-g}\left(R-G_{i j} \partial_{\mu} \phi^{i} \partial_{\mu} \phi^{j}\right)-\stackrel{\circ}{a}_{A B} F^{A} \wedge \star F^{B}+\frac{1}{24} \int C_{A B C} A^{A} \wedge F^{B} \wedge F^{C}
$$

where the Chern-Simons-type couplings $C_{A B C}$ are constant, for gauge invariance. $\mathcal{N}=$ 2 supersymmetry requires the real scalar fields $\phi^{i}$ to take value in the cubic hypersurface $\mathcal{M}_{5}=\{\xi, N(\xi)=1\}$, where

$$
N(\xi)=\frac{1}{6} C_{A B C} \xi^{A} \xi^{B} \xi^{C}
$$

The metric $G_{i j}$ is then the pull-back of the ambiant space metric $a_{A B} d \xi^{A} d \xi^{B}$ to $\mathcal{M}_{5}$, where

$$
a_{A B}=-\frac{1}{2} \partial_{\xi^{A}} \partial_{\xi^{B}} N(\xi)
$$

The gauge couplings $\stackrel{\circ}{a}_{A B}$ are similarly the restriction of $a_{A B}$ to the hypersurface $\mathcal{M}_{5}$.

Upon reduction from 5 dimensions to 4 dimensions, using the standard KaluzaKlein ansatz

$$
d s_{5}^{2}=e^{2 \sigma}\left(d y+B_{\mu} d x^{\mu}\right)^{2}+e^{-\sigma} g_{\mu \nu} d x^{\mu} d x^{\nu}
$$

the Kaluza-Klein gauge field $B_{\mu}$ provides the graviphoton, while the constraint $N(\xi)=$ 1 is relaxed to $N(\xi)=e^{3 \sigma}$. Moreover, $\xi^{I}$ combine with the fifth components $a^{I}$ of the gauge fields $A_{M}^{I}$ into complex scalars $t^{I}=a^{I}+i \xi^{I}=X^{I} / X^{0}$, which are the special coordinates of a special Kähler manifold $\mathcal{M}_{4}$ with prepotential

$$
F=N\left(X^{A}\right) / X^{0}
$$

In general, neither $\mathcal{M}_{5}$ nor $\mathcal{M}_{4}$ are symmetric spaces. The conditions for $\mathcal{M}_{5}$ to be a symmetric space were analyzed in [43], and found to have a remarkably simple interpretation in terms of Jordan algebras: these are commutative, non-associative algebras $J$ satisfying the "Jordan identity"

$$
x \circ\left(y \circ x^{2}\right)=(x \circ y) \cdot x^{2}
$$

where $x^{2}=x \circ x$ (see [44] for a nice review). 
Exercice 8 Show that the algebra of $n \times n$ hermitean matrices with product $A \circ B=$ $\frac{1}{2}(A B+B A)$ is a Jordan algebra.

Jordan algebras were introduced and completely classified in [45] in an attempt to generalize quantum mechanics beyond the field of complex numbers. The ones relevant to us are those which admit a norm $N$ of degree 3 - rather than giving the axioms of the norm, we shall merely list the allowed possibilities:

i) One trivial case: $J=\mathbb{R}, N(\xi)=\xi^{3}$

ii) One infinite series: $J=\mathbb{R} \oplus \Gamma$ where $\Gamma$ is the Clifford algebra of $O(1, n-1)$, $N(\xi \oplus \gamma)=\xi \gamma^{a} \gamma^{b} \eta_{a b}$

iii) Four exceptional cases: $J=\operatorname{Herm}_{3}(\mathbb{D})$, the algebra of $3 \times 3$ hermitean matrices $\xi=\left(\begin{array}{lll}\alpha_{1} & x_{3} & \bar{x}_{2} \\ \bar{x}_{3} & \alpha_{2} & x_{1} \\ x_{2} & \bar{x}_{1} & \alpha_{3}\end{array}\right)$ where $\alpha_{i}$ are real and $x_{i}$ are in one of the four "division algebras" $\mathbb{D}=\mathbb{R}, \mathbb{C}$, the quaternions $\mathbb{H}$ or octonions $\mathbb{O}$. In each of these cases, the cubic norm is the "determinant" of $\xi$

$$
N(\xi)=\alpha_{1} \alpha_{2} \alpha_{3}-\alpha_{1} x_{1} \bar{x}_{1}-\alpha_{2} x_{2} \bar{x}_{2}-\alpha_{3} x_{3} \bar{x}_{3}+2 \operatorname{Re}\left(x_{1} x_{2} x_{3}\right)
$$

For $J_{3}^{\mathbb{C}}$, it is equivalent to the determinant of an unconstrained $3 \times 3$ real matrix, and for $J_{3}^{\mathbb{H}}$ to the Pfaffian of a $6 \times 6$ antisymmetric matrix.

To each of these Jordan algebras, one may attach several invariance groups, summarized in Table 1:

a) $\operatorname{Aut}(J)$, the group of automorphisms of $J$, which leaves invariant the structure constants of the Jordan product;

b) $\operatorname{Str}(J)$, the "structure" group, which leaves invariant the norm $N(\xi)$ up to a rescaling; and the "reduced structure group" $\operatorname{Str}_{0}(J)$, where the center has been divided out;

c) $\operatorname{Conf}(J)$, the "conformal" group, such that the norm of the difference of two elements $N\left(\xi-\xi^{\prime}\right)$ is multiplied by a product $f(\xi) f\left(\xi^{\prime}\right)$; as a result, the "cubic light-cone" $N\left(\xi-\xi^{\prime}\right)=0$ is invariant;

d) QConf $(J)$, the "quasi-conformal group", which we will describe in Section 7.4. 


\begin{tabular}{|c|c|c|c|c|}
\hline$J$ & $\operatorname{Aut}(J)$ & $\operatorname{Str}_{0}(J)$ & $\operatorname{Conf}(J)$ & $\mathrm{QConf}(J)$ \\
\hline $\mathbb{R}$ & 1 & 1 & $S l(2, \mathbb{R})$ & $G_{2(2)}$ \\
\hline $\mathbb{R} \oplus \Gamma_{n-1,1}$ & $S O(n-1)$ & $S O(n-1,1)$ & $S l(2) \times S O(n, 2)$ & $S O(n+2,4)$ \\
\hline$J_{3}^{\mathbb{R}}$ & $S O(3)$ & $S l(3, \mathbb{R})$ & $S p(6)$ & $F_{4(4)}$ \\
\hline$J_{3}^{\mathbb{C}}$ & $S U(3)$ & $S l(3, \mathbb{C})$ & $S U(3,3)$ & $E_{6(+2)}$ \\
\hline$J_{3}^{\mathbb{H}}$ & $U S p(6)$ & $S U^{*}(6)$ & $S O^{*}(12)$ & $E_{7(-5)}$ \\
\hline$J_{3}^{\bigotimes}$ & $F_{4}$ & $E_{6(-26)}$ & $E_{7(-25)}$ & $E_{8(-24)}$ \\
\hline
\end{tabular}

Table 1: Invariance groups associated to degree 3 Jordan algebras. The lower $4 \times 4$ part is known as the "Magic Square", due to its symmetry along the diagonal [46].

In the case ii) above, $\operatorname{Aut}(J), \operatorname{Str}(J)$ and $\operatorname{Conf}(J)$ are just the orthogonal group $S O(n-1)$, Lorentz group $S O(n-1,1)$ and conformal group $S O(n, 2)$ times an extra $S l(2)$ factor.

The relevance of these groups for physics is as follows: choosing $N(\xi)$ in (3.52) to be equal to the norm form of a Jordan algebra $J$, the vector-multiplet moduli spaces for the resulting $\mathcal{N}=2$ supergravity in $D=5$ and $D=4$ are symmetric spaces

$$
\mathcal{M}_{5}=\frac{\operatorname{Str}_{0}(J)}{\operatorname{Aut}(J)}, \quad \mathcal{M}_{4}=\frac{\operatorname{Conf}(J)}{\operatorname{Str}_{0}(J) \times U(1)}
$$

where $\widetilde{\operatorname{Str}}_{0}(J)$ denotes the compact real form of $\operatorname{Str}_{0}(J)$. In either case, the group in the denominator is the maximal subgroup of the one in the numerator, which guarantees that the quotient has positive definite signature. The resulting spaces are shown in Table 2, together with the ones which appear upon reduction to $D=3$ on a space-like and time-like direction respectively, which we will discuss in Section 7.4 below. The first column indicates the number of supercharges in the corresponding supergravity: the above discussion applies strictly speaking to cases with 8 supercharges (i.e. $\mathcal{N}=2$ supersymmetry in 4 dimensions), but other cases can also be reached with similar techniques, using different real forms of the Jordan algebras above ${ }^{8}$.

The $\operatorname{Str}_{0}(J)$ invariance of the metric on $\mathcal{M}_{5}$ is indeed obvious from (3.53) above. The $\operatorname{Conf}(J)$ invariance of the metric on the special Kähler space $\mathcal{M}_{4}$ is manifest too, since the Kähler potential following from (3.55) is the "cubic light-cone"

$$
K(z, \bar{z})=-\log N\left(z_{i}-\bar{z}_{i}\right)
$$

${ }^{8}$ For example, the cubic invariant of $E_{6(6)}$ appearing in $\mathcal{N}=8$ supergravity can be obtained from (3.57) by replacing the usual octonions $\mathbb{O}$ by the split octions $\mathbb{O}_{s}$, whose norm $x \bar{x}$ has split signature $(4,4)$, see $[47]$ for a recent discussion. 


\begin{tabular}{|c|c|c|c|c|c|}
\hline$Q$ & $J$ & $D=5$ & $D=4$ & $D=3$ & $D=3^{*}$ \\
\hline 8 & & & $\frac{S U(n, 1)}{S U(n) \times U(1)}$ & $\frac{S U(n+1,2)}{S U(n+1) \times S U(2) \times U(1)}$ & $\frac{S U(n+1,2)}{S U(n, 1) \times S l(2) \times U(1)}$ \\
\hline 8 & $\Gamma_{n-1,1}$ & $\mathbb{R} \times \frac{S O(n-1,1)}{S O(n-1)}$ & $\frac{S O(n, 2)}{S O(n) \times S O(2)} \times \frac{S l(2)}{U(1)}$ & $\frac{S O(n+2,4)}{S O(n+2) \times S O(4)}$ & $\frac{S O(n+2,4)}{S O(n, 2) \times S O(2,2)}$ \\
\hline 8 & & & $\frac{S l(2)}{U(1)}$ & $\frac{S U(2,1)}{S U(2) \times U(1)}$ & $\frac{S U(2,1)}{S l(2) \times U(1)}$ \\
\hline 8 & $\mathbb{R}$ & $\varnothing$ & $\frac{S l(2)}{U(1)}$ & $\frac{G_{2(2)}}{S O(4)}$ & $\frac{G_{2(2)}}{S O(2,2)}$ \\
\hline 8 & $J_{3}^{\mathbb{R}}$ & $\frac{S l(3)}{S O(3)}$ & $\frac{S p(6)}{S U(3) \times U(1)}$ & $\frac{F_{4(4)}}{U S p(6) \times S U(2)}$ & $\frac{F_{4(4)}}{S p(6) \times S l(2)}$ \\
\hline 8 & $J_{3}^{\mathbb{C}}$ & $\frac{S l(3, C)}{S U(3)}$ & $\frac{S U(3,3)}{S U(3) \times S U(3) \times U(1)}$ & $\frac{E_{6(+2)}}{S U(6) \times S U(2)}$ & $\frac{E_{6(+2)}}{S U(3,3) \times S l(2)}$ \\
\hline 24 & $J_{3}^{\mathbb{H}}$ & $\frac{S U^{*}(6)}{U S p(6)}$ & $\frac{S O^{*}(12)}{S U(6) \times U(1)}$ & $\frac{E_{7(-5)}}{S O(12) \times S U(2)}$ & $\frac{E_{7(-5)}}{S O^{*}(12) \times S l(2)}$ \\
\hline 8 & $J_{3}^{\mathbb{Q}}$ & $\frac{E_{6(-26)}}{F_{4}}$ & $\frac{E_{7(-25)}}{E_{6} \times U(1)}$ & $\frac{E_{8(-24)}}{E_{7} \times S U(2)}$ & $\frac{E_{8(-24)}}{E_{7(-25) \times S l(2)}}$ \\
\hline 10 & & & $\frac{S p(2 n, 4)}{S p(2 n) \times S p(4)}$ & \\
\hline 12 & & & $\frac{S U(n, 4)}{S U(n) \times S U(4)}$ & \\
\hline 16 & $\Gamma_{n-5,5}$ & $\mathbb{R} \times \frac{S O(n-5,5)}{S O(n-5) \times S O(5)}$ & $\frac{S l(2)}{U(1)} \times \frac{S O(n-4,6)}{S O(n-4) \times S O(6)}$ & $\frac{S O(n-2,8)}{S O(n-2) \times S O(8)}$ & $\frac{S O(n-2,8)}{S O(n-4,2) \times S O(2,2)}$ \\
\hline 18 & & & & $\frac{F_{4(-20)}}{S O(9)}$ & \\
\hline 20 & $M_{1,2}(\mathbb{O})$ & & $\frac{S U(5,1)}{S U(5) \times U(1)}$ & $\frac{E_{6(-14)}}{S O(10) \times S O(2)}$ & $\frac{E_{6(-14)}}{S O^{*}(10) \times S O(2)}$ \\
\hline 32 & $J_{3}^{\mathbb{Q}}$ & $\frac{E_{6(6)}}{U S p(8)}$ & $\frac{E_{7(7)}}{S U(8)}$ & $\frac{E_{8(8)}}{S O(16)}$ & $\frac{E_{8(8)}}{S O^{*}(16)}$ \\
\hline
\end{tabular}

Table 2: Moduli spaces for supergravities with symmetric moduli spaces. The last column refers to the reduction from 4 dimensions to 3 along a time-like direction.

invariant under Conf $(J)$ up to Kähler transformations. Such special Kähler spaces are known as hermitean symmetric tube domains, and are higher-dimensional analogues of Poincaré's upper half plane.

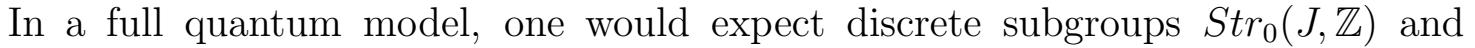
$\operatorname{Conf}(J, \mathbb{Z})$ to remain as quantum symmetries of the theory. We shall refer to these symmetries as "U-dualities".

It should be pointed out that there also exist $D=4$ SUGRAs with symmetric moduli space which do not descend from 5 dimensions: they may be described by a generalization of Jordan algebras known as "Freudenthal triple systems", but we will not discuss them in any detail here. Similarly, there exist $D=3$ supergravity with symmetric moduli spaces which cannot be lifted to 4 dimensions.

\subsection{Bekenstein-Hawking Entropy in Very Special Supergravities}

As an illustration of the simplicity of these models, we shall now proceed and compute the Bekenstein-Hawking entropy for BPS black holes with arbitrary charges, following 
[48]. A key property which renders the computation tractable is the fact that the prepotential (3.55) obtained from Jordan algebras is invariant (up to a sign) under Legendre transform in all variables, namely

$$
\left\langle N\left(X^{A}\right) / X^{0}+X^{A} Y_{A}+X^{0} Y_{0}\right\rangle_{X^{I}}=-N(Y) / Y^{0}
$$

Exercice 9 Show that (3.60) is equivalent to the "adjoint identity" for Jordan algebras, $X^{\sharp \sharp}=N(X) X$ where $X_{A}^{\sharp}=\frac{1}{2} C_{A B C} X^{B} X^{C}$ is the "quadratic map" from $J$ to its dual.

In fact, just imposing (3.60) leads to the same classification i),ii),iii) as above. This was shown independently in [49], as a first step in finding cubic analogues of the Gaussian, invariant under Fourier transform (see [50] for a short account).

Exercice 10 Check by explicit computation that for the "STU" model, $\left(1 / X^{0}\right) e^{N\left(X^{A}\right) / X^{0}}$ is invariant under Fourier transform, namely

$$
\int \frac{d X^{0} d X^{1} d X^{2} d X^{3}}{X^{0}} \exp \left[i \frac{X^{1} X^{2} X^{3}}{\hbar X^{0}}+i X^{I} Y_{I}\right]=\frac{\hbar}{Y^{0}} \exp \left[i \hbar \frac{Y_{1} Y_{2} Y_{3}}{Y_{0}}\right]
$$

Conclude that the semi-classical approximation to this integral is exact.

In order to compute the Bekenstein-Hawking entropy, we start from the "free energy" (3.47)

$$
\mathcal{F}(p, \phi)=\frac{\pi}{\left(p^{0}\right)^{2}+\left(\phi^{0}\right)^{2}}\left\{p^{0}\left[\phi^{A} p_{A}^{\sharp}-N(\phi)\right]+\phi^{0}\left[p^{A} \phi_{A}^{\sharp}-N(p)\right]\right\}
$$

To eliminate the quadratic term in $\phi^{A}$, let us change variables to

$$
x^{A}=\phi^{A}-\frac{\phi^{0}}{p^{0}} p^{A}, \quad x^{0}=\left[\left(p^{0}\right)^{2}+\left(\phi^{0}\right)^{2}\right] / p^{0}
$$

Moreover, we introduce an auxiliary variable $t$, such that, upon eliminating $t$, we recover (3.62):

$$
S_{B H}=\pi\left\langle-\frac{N\left(x^{A}\right)}{x^{0}}+\frac{p_{A}^{\sharp}+p^{0} q_{A}}{p^{0}} x^{A}-\frac{t}{4}\left(\frac{x^{0}}{p^{0}}-1\right)-\frac{\left(2 N(p)+p^{0} p^{I} q_{I}\right)^{2}}{t\left(p^{0}\right)^{2}}\right\rangle_{\left\{x^{I}, t\right\}}
$$

Extremizing over $x^{I}$ now amounts to Legendre transforming $N(x) / x^{0}$, which according to (3.60) reproduces $-N(y) / y^{0}$ where $y^{I}$ are the coefficients of the linear terms in $x^{I}$, so

$$
S_{B H}=\pi\left\langle 4 \frac{N\left[p_{A}^{\sharp}+p^{0} q_{A}\right]}{\left(p^{0}\right)^{2} t}-\frac{\left[2 N(p)+p^{0} p^{I} q_{I}\right]^{2}}{t\left(p^{0}\right)^{2}}-\frac{t}{4}\right\rangle_{t}
$$


Finally, extremizing over $t$ leads to

$$
S_{B H}=\frac{\pi}{p^{0}} \sqrt{4 N\left[p_{A}^{\sharp}+p^{0} q_{A}\right]-\left[2 N(p)+p^{0} p^{I} q_{I}\right]^{2}}
$$

The pole at $p^{0}=0$ is fake, since upon Taylor expanding $N\left[p_{A}^{\sharp}+p^{0} q_{A}\right]$ in the numerator and further using the homogeneity of $N$, its coefficient cancels. The final result gives the entropy as the square root of a quartic polynomial in the charges,

$$
S_{B H}=\pi \sqrt{I_{4}\left(p^{I}, q_{I}\right)}
$$

where

$$
I_{4}(p, q)=4 p^{0} N(q)-4 q_{0} N(p)+4 q_{\sharp}^{A} p_{A}^{\sharp}-\left(p^{0} q_{0}+p^{A} q_{A}\right)^{2}
$$

The fact that this quartic polynomial is invariant under the linear action of the fourdimensional "U-duality" group $\operatorname{Conf}(J)$ on the symplectic vector of charges $\left(p^{I}, q_{I}\right)$, follows from Freudenthal's "triple system construction". Several examples are worth mentioning:

- For the "STU" model with $N(\xi)=\xi^{1} \xi^{2} \xi^{3}$, the electric-magnetic charges transform as a $(2,2,2)$ of $\operatorname{Conf}(J)=S l(2)^{3}$, so can be viewed as sitting at the 8 corners of a cube; the quartic invariant is known as Cayley's "hyperdeterminant"

$$
I_{4}=-\frac{1}{2} \epsilon^{A B} \epsilon^{C D} \epsilon^{a b} \epsilon^{c d} \epsilon^{\alpha \gamma} \epsilon^{\beta \delta} Q_{A a \alpha} Q_{B b \beta} Q_{C c \gamma} Q_{D d \delta}
$$

This has recently been related to the "three-bit entanglement" in quantum information theory ${ }^{9}[51,52]$.

- More generally, for the infinite series, where the charges transform as a $(2, n)$ of $S l(2) \times S O(2, n)$, the quartic invariant is

$$
I_{4}=\left(\vec{Q}_{e} \cdot \vec{Q}_{e}\right)\left(\vec{Q}_{m} \cdot \vec{Q}_{m}\right)-\left(\vec{Q}_{e} \cdot \vec{Q}_{m}\right)^{2}
$$

Up to a change of signature of the orthogonal group, this is the quartic invariant which appears in the entropy of $1 / 4$-BPS black holes in $\mathcal{N}=4$ theories (2.22).

\footnotetext{
${ }^{9}$ According to Freudenthal's construction, the electric and magnetic charges naturally form a square (rather than a cube) $\left(\begin{array}{ll}p^{0} & p^{I} \\ q_{I} & q_{0}\end{array}\right)$, where the diagonal elements are in $\mathbb{R}$ while the off-diagonal ones are in the Jordan algebra $J$. This suggests that the "three-bit" interpretation of the STU model may be difficult to generalize.
} 
- In the exceptional $J_{3}^{\mathbb{Q}}$ case, $I_{4}$ is the quartic invariant of the 56 representation of $E_{7(-25)}$. Replacing $\mathbb{O}$ by the split octonions $\mathbb{O}_{s}$, one obtains the quartic invariant of $E_{7(7)}$, which appears in the entropy $S=\pi \sqrt{I_{4}}$ of $1 / 8$-BPS states in $\mathcal{N}=8$ supergravity [53],

$$
I_{4}(P, Q)=-\operatorname{Tr}(Q P Q P)+\frac{1}{4}(\operatorname{Tr} Q P)^{2}-4[\operatorname{Pf}(P)+\operatorname{Pf}(Q)]
$$

where the entries in the antisymmetric $8 \times 8$ matrices $Q$ and $P$ may be identified as [48]:

$$
Q=\left(\begin{array}{ccc}
{[D 2]^{i j}} & {[F 1]^{i}} & {[k k m]^{i}} \\
-[F 1]^{i} & 0 & {[D 6]} \\
-[k k m]^{i} & -[D 6] & 0
\end{array}\right), \quad P=\left(\begin{array}{ccc}
{[D 4]_{i j}} & {[N S 5]_{i}} & {[k k]_{i}} \\
-[N S 5]_{i} & 0 & {[D 0]} \\
-[k k]_{i} & -[D 0] & 0
\end{array}\right)
$$

Here, $[D 2]^{i j}$ denotes a D2-brane wrapped along the directions $i j$ on $T^{6},[D 4]_{i j}$ a D4-branes wrapped on all directions but $i j,[k k]_{i}$ a momentum state along direction $i,[\mathrm{kkm}]^{i}$ a Kaluza-Klein 5-monopole localized along the direction $i$ on $T^{6},[F 1]^{i}$ a fundamental string winding along direction $i$, and $[N S 5]_{i}$ a NS5-brane wrapped on all directions but $i$.

Exercice 11 Show that in the $\mathcal{N}=4$ truncation where only the $[F 1],[k k],[N S 5],[k k m]$ charges are retained, (3.71) reduces to the quartic invariant (2.22) under $S l(2) \times$ $S O(6,6)$. Similarly, in the $\mathcal{N}=2$ truncation where only $[D 0],[D 2],[D 4],[D 6]$ are kept, show that one obtains the quartic invariant of a spinor of $S O^{*}(12)$, based on the Jordan algebra $J_{3}^{\mathbb{H}}$.

The intermediate equation (3.65) also has an interesting interpretation: it is recognized as $1 / p^{0}$ times the entropy $S_{5 D}=\pi \sqrt{N(Q)-J^{2}}$ of a five-dimensional BPS black hole with electric charge and angular momentum

$$
\begin{aligned}
Q_{A} & =p^{0} q_{A}+C_{A B C} p^{B} p^{C} \\
2 J_{L} & =\left(p^{0}\right)^{2} q_{0}+p^{0} p^{A} q_{A}+2 N(p)
\end{aligned}
$$

Indeed, when the D6-brane charge $p^{0}$ is non-zero, the $4 \mathrm{D}$ black hole in Type IIA compactified on $\tilde{Y}$ may be lifted to a $5 \mathrm{D}$ black hole in M-theory on $\tilde{Y} \times T N_{p^{0}}$, where $T N$ denotes the 4-dimensional Euclidean Taub-NUT space with NUT charge $p^{0}$; at spatial infinity, this asymptotes to $\mathbb{R}^{3} \times S^{1}$, where the circle is taken to be the M-theory direction. Translations along this direction at infinity, conjugate to the D0-brane charge $q_{0}$, become $S U(2)$ rotations at the center of $T N$, where the black hole is assumed to sit. The remaining factors of $p^{0}$ are accounted for by taking into account the $\mathbb{R}^{4} / \mathbb{Z}_{p^{0}}$ singularity at the origin of $T N$ [54]. The formulae (3.73) extend this lift to an arbitrary choice of charges, in a manifestly duality invariant manner. 
Exercice 12 Using the fact that the degeneracies of five-dimensional black holes on $K 3 \times S^{1}$ are given by the Fourier coefficients of the elliptic genus of Hilb(K3), equal to $1 / \Phi_{10}$, show that the $D V V$ conjecture (2.23) holds for at least one $U$-duality orbit of 4-dimensional dyons in type II $/ K 3 \times T^{2}$ with one unit of D6-brane and some amout of D0,D2-brane charge. You might want to seek help from [27].

\section{Topological String Primer}

In the previous sections, we were concerned exclusively with low energy supergravity theories, whose Lagrangian contains at most two-derivative terms. This is sufficient in the limit of infinitely large charges, but not for more moderate values, where higherderivative corrections start playing a role. In this section, we give a self-contained introduction to topological string theory, which offers a practical way of to compute an infinite series of such corrections. Sections 4.1 and 4.2 draw heavily from [55]. Other valuable reviews of topological string theory include [56, 57, 58, 59, 60].

\subsection{Topological Sigma Models}

Type II strings compactified on a Kähler manifold $X$ of complex dimension $d$ are described by a $N=(2,2)$ sigma model

$$
S=2 t \int d^{2} z\left(g_{i \bar{j}} \partial \phi^{i} \bar{\partial} \phi^{\bar{j}}+g_{i \bar{j}} \bar{\partial} \phi^{i} \partial \phi^{\bar{j}}+i \psi_{-}^{\bar{i}} D \psi_{-}^{i} g_{i \bar{i}}+i \psi_{+}^{\bar{i}} \bar{D} \psi_{+}^{i} g_{i \bar{i}}+R_{i \bar{i} \bar{j}} \psi_{+}^{i} \psi_{+}^{\bar{i}} \psi_{-}^{j} \psi_{-}^{\bar{j}}\right)
$$

where $\phi$ is a map from a two-dimensional genus $g$ Riemann surface $\Sigma$ to $X, \psi_{ \pm}^{i}$ is a section of $K_{ \pm}^{1 / 2} \otimes \phi^{*}\left(T^{1,0} X\right), \psi_{ \pm}^{\bar{i}}$ is a section of $K_{ \pm}^{1 / 2} \otimes \phi^{*}\left(T^{0,1} X\right)$, and we denoted by $K_{+}$ the canonical bundle on $\Sigma$ (i.e. the bundle of $(1,0)$ forms) and $K_{-}$the anti-canonical bundle (of $(0,1)$ forms). The factor of $t$ (the string tension) is to keep track on the dependence on the overall volume of $X$.

This model is invariant under $N=(2,2)$ superconformal transformations generated with sections $\alpha_{ \pm}$and $\tilde{\alpha}_{ \pm}$of $K_{ \pm}^{1 / 2}$, acting e.g. as

$$
\delta \phi^{i}=i\left(\alpha_{-} \psi_{+}^{i}+\alpha_{+} \psi_{-}^{i}\right), \quad \delta \phi^{\bar{i}}=i\left(\tilde{\alpha}_{-} \psi_{+}^{\bar{i}}+\tilde{\alpha}_{+} \psi_{-}^{\bar{i}}\right)
$$

This implies chirally conserved supercurrents $G^{ \pm}$of conformal dimension 3/2, which together with $T$ and the current $J$ generate the $\mathcal{N}=2$ superconformal algebra,

$$
\begin{aligned}
G^{+}(z) G^{-}(0) & =\frac{2 c}{3} \frac{1}{z^{2}}+\left(\frac{2 J}{z^{2}}+\frac{\partial J+2 T}{z}\right)+\text { reg } \\
J(z) J(0) & =\frac{c}{3} \frac{1}{z^{2}}+\text { reg }
\end{aligned}
$$


The current $J$ appearing in the OPE (4.3) generates a $U(1)$ symmetry, such that $G_{ \pm}$ have charge $Q= \pm 1$ while $T$ and $J$ are neutral. In the (doubly degenerate) Ramond sectors $R_{ \pm}$, the zero-modes of the supercurrents generate a supersymmetry algebra

$$
\left(G_{0}^{+}\right)^{2}=\left(G_{0}^{-}\right)^{2}=0, \quad\left\{G_{0}^{+}, G_{0}^{-}\right\}=2\left(L_{0}^{R_{ \pm}}-\frac{c}{24}\right)
$$

Unitarity forces the right-hand side to be positive on any state. Moreover, the $\mathcal{N}=2$ algebra admits an automorphism known as spectral flow, which relates the NS and R sectors:

$$
J_{0}^{R_{ \pm}}=J_{0}^{N S} \mp \frac{c}{6}, \quad L_{0}^{R_{ \pm}}=L_{0}^{N S} \mp \frac{1}{2} J_{0}^{N S}+\frac{c}{24}
$$

The unitary bound $\Delta \geq c / 24$ in the R sector therefore implies a bound $\Delta \geq|Q| / 2$ after spectral flow. States which saturate this bound have no short distance singularities when brought together, and thus form a ring under OPE, known as the chiral ring of the $\mathcal{N}=2$ SCFT. Applying the spectral flow twice maps the NS sector back to itself, with $(\Delta, Q) \rightarrow\left(\Delta-Q+\frac{c}{6}, Q \mp \frac{c}{3}\right)$. In particular, the NS ground state is mapped to a state with $(\Delta, q)=\left(\frac{c}{6}, \mp \frac{c}{3}\right)$ in the chiral ring. For a Calabi-Yau three-fold, starting from the identity we thus obtain two $\mathrm{R}$ states with $(\Delta, q)=(3 / 8, \pm 3 / 2)$, and one NS state with $(\Delta, q)=(3 / 2, \pm 3)$ : these are identified geometrically as the covariantly constant spinor and the holomorphic $(3,0)$ form, respectively.

The spectral flow (4.6) above can be used to "twist" the $\mathcal{N}=2$ sigma model into a topological sigma model: for this, bosonize the $U(1)$ current $J=i \sqrt{3} \partial H$, so that the spectral flow operator becomes

$$
\Sigma_{ \pm}=\exp \left( \pm i \frac{\sqrt{3}}{2} H(z)\right)
$$

with $(\Delta=3 / 8, Q= \pm 3 / 2)$. The topological twist then amounts to adding a background charge $\pm \int \frac{\sqrt{3}}{2} H R^{(2)}$ : its effect is to change the two-dimensional spin $L_{0}$ into a linear combination $L_{0} \mp \frac{1}{2} J_{0}$ of the spin and the $U(1)$ charge. Under this operation, choosing the + sign, $\psi_{+}^{i}$ becomes a section of $\phi^{*}\left(T^{1,0} X\right)$, i.e. a worldsheet scalar, whereas $\psi_{+}^{\bar{i}}$ becomes a section of $K_{+} \otimes \phi^{*}\left(T^{0,1} X\right)$, i.e. a worldsheet one-form; simultaneously, the supersymmetry parameters $\alpha_{-}$and $\tilde{\alpha}_{-}$become a scalar and a section of $K^{-1}$, respectively. Alternatively, we may choose the $-\operatorname{sign}$ in (4.6), where instead $\psi_{+}^{i}$ would become a section of $K_{+} \otimes \phi^{*}\left(T^{1,0} X\right)$, while $\psi_{+}^{\bar{i}}$ would turn into a worldsheet scalar. In either case, it is necessary that the canonical bundle $K$ be trivial, in order for that the correlation functions be unaffected by the twist : this is achieved only when computing particular "topological amplitudes" in string theory, which we will discuss in Section 5.1 . 
Since the sigma model $(4.1)$ has $(2,2)$ superconformal invariance, it is possible to twist both left and right-movers by a spectral flow of either sign. Only the relative choice of sign is important, leading to two very distinct-looking theories, which we discuss in turn:

\subsubsection{Topological A-model}

Here, both $\psi_{+}^{i}$ and $\psi_{-}^{\bar{i}}$ are worldsheet scalars, and can be combined in a scalar $\chi \in$ $\phi^{*}(T X)$. On the other hand, $\psi_{-}^{i}$ and $\psi_{+}^{\bar{i}}$ become $(0,1)$ and $(1,0)$ forms $\psi_{\bar{z}}^{i}$ and $\psi_{z}^{\bar{i}}$ on the worldsheet. The action is rewritten as

$$
S=2 t \int d^{2} z\left(g_{i \bar{j}} \partial \phi^{i} \bar{\partial} \phi^{\bar{j}}+g_{i \bar{j}} \bar{\partial} \phi^{i} \partial \phi^{\bar{j}}+i \psi_{z}^{\bar{i}} \bar{D} \chi^{i} g_{i \bar{i}}+i \psi_{\bar{z}}^{\bar{i}} D \chi^{\bar{i}} g_{i \bar{i}}-R_{i \bar{i} j \bar{j}} \psi_{\bar{z}}^{i} \psi_{z}^{\bar{i}} \chi^{j} \chi^{\bar{j}}\right)
$$

It allows for a conserved "ghost" charge where $[\phi]=0,[\chi]=1,[\psi]=-1$, and is invariant under the scalar nilpotent operator $Q=G_{+}$,

$$
\left\{Q, \phi^{I}\right\}=\chi^{I}, \quad\left\{Q, \chi^{I}\right\}=0,\left\{Q, \psi_{\bar{z}}^{i}\right\}=i \bar{\partial} \phi^{i}-\chi^{j} \Gamma_{j k}^{i} \psi_{\bar{z}}^{k}
$$

The action (4.8) is in fact $Q$-exact, up to a total derivative term proportional to the pull-back of the Kähler form $\omega_{K}=i g_{i \bar{j}} d \phi^{i} \wedge d \phi^{\bar{j}}$, complexified into $J=B+i \omega_{K}$ by including the coupling to the NS two-form:

$$
S=-i\{Q, V\}-t \int_{\Sigma} \phi^{*}(J)
$$

where $V$ is the "gauge fermion"

$$
V=t \int d^{2} z g_{i \bar{j}}\left(\psi_{\bar{z}}^{i} \partial \phi^{\bar{j}}+\psi_{z}^{\bar{j}} \bar{\partial} \phi^{i}\right)
$$

This makes it clear that the theory is independent of the worldsheet metric, since the energy momentum tensor is $Q$-exact:

$$
T_{\alpha \beta}=\left\{Q, b_{\alpha \beta}\right\}, \quad b_{\alpha \beta}=\frac{\partial V}{\partial g^{\alpha \beta}}
$$

Moreover, the string tension $t$ appears only in the total derivative term so, in a sector with fixed homology class $\int_{\Sigma} \phi^{*}(J)$, the semi-classical limit $t \rightarrow 0$ is exact. The path integral thus localizes ${ }^{10}$ to the moduli space of $Q$-exact configurations,

$$
\partial_{\bar{z}} \phi^{i}=0, \quad \partial_{z} \phi^{\bar{i}}=0
$$

\footnotetext{
${ }^{10}$ Localization is a general feature of integrals with a fermionic symmetry $Q$ : decompose the space of fields into orbits of $Q$, parameterized by a Grassman variable $\theta$, times its orthogonal complement; since the integrand is independent of $\theta$ by assumption, the integral $\int d \theta$ vanishes by the usual rules of Grassmannian integration. This reasoning breaks down at the fixed points of $Q$, which is the locus to which the integral localizes.
} 
i.e. holomorphic maps from $\Sigma$ to $X$. Moreover, the local observables of the A-model $O_{\mathcal{W}}=W_{I_{1} \ldots I_{n}} \chi^{I_{1}} \ldots \chi^{I_{n}}$, where $W_{I_{1} \ldots I_{n}} d \phi^{I_{1}} \ldots d \phi^{I_{n}}$ is a differential form on $X$ of degree $n$, are in one-to-one correspondence with the de Rham cohomology of $X$, since $\left\{Q, \mathcal{O}_{W}\right\}=-\mathcal{O}_{d W}$. Due to an anomaly in the conservation of the ghost charge, correlators of $l$ observables vanish unless

$$
\sum_{k=1}^{l} \operatorname{deg}\left(W_{k}\right)=2 d(1-g)+2 \int_{\Sigma} \phi^{*}\left(c_{1}(X)\right)
$$

The last term vanishes when the Calabi-Yau condition $c_{1}(X)$ is obeyed. For Calabi-Yau threefolds, at genus 0 the only correlator involves three degree 2 forms,

$$
\left\langle\mathcal{O}_{W_{1}} \mathcal{O}_{W_{2}} \mathcal{O}_{W_{3}}\right\rangle=\int W_{1} \wedge W_{2} \wedge W_{3}+\sum_{\beta \in H^{2+}(X)} e^{-t \int_{\beta} J} \int_{\beta} W_{1} \int_{\beta} W_{2} \int_{\beta} W_{3}
$$

At genus 1, only the vacuum amplitude, known as the elliptic genus of $X$ is non-zero. In Section 4.2, we will explain the prescription to construct non-zero amplitudes at any genus, by coupling to topological gravity.

\subsubsection{Topological B-model}

The other inequivalent choice consists in twisting $\psi_{ \pm}^{\bar{i}}$ into worldsheet scalars valued in $T X^{0,1}$, while $\psi_{+}^{i}$ and $+\psi_{-}^{i}$ are $(0,1)$ and $(1,0)$ forms valued in $T X^{1,0}$. Defining $\eta^{\bar{i}}=\psi_{+}^{\bar{i}}+\psi_{-}^{\bar{i}}, \theta_{i}=g_{i \bar{i}}\left(\psi_{+}^{\bar{i}}-\psi_{-}^{\bar{i}}\right.$, and taking $\psi_{ \pm}^{i}$ as the two components of a one-form $\rho^{i}$, the action may be rewritten as

$$
S=i t\{Q, V\}+t W
$$

where

$$
\begin{aligned}
V & =\int_{\Sigma} d^{2} z g_{i \bar{j}}\left(\rho_{z}^{i} \bar{\partial} \phi^{\bar{j}}+\rho_{z}^{i} \bar{\partial} \phi^{\bar{j}}\right) \\
W & =-\int_{\Sigma} d^{2} z\left(\theta_{i} D \rho^{i}+\frac{i}{2} R_{i \bar{i} j \bar{j}} \rho^{i} \wedge \rho^{j} \eta^{\bar{i}} \theta_{k} g^{k \bar{j}}\right)
\end{aligned}
$$

and the nilpotent operator $Q=G_{-}$acts as

$$
\left\{Q, \phi^{i}\right\}=0, \quad\left\{Q, \phi^{\bar{i}}\right\}=-\eta^{\bar{i}}, \quad\left\{Q, \eta^{\bar{i}}\right\}=\left\{Q, \theta_{i}\right\}=0, \quad\left\{Q, \rho^{i}\right\}=-i d \phi^{i}
$$

Again, the energy-momentum tensor is $Q$-exact, so that the model is topological. It is also independent of the Kähler structure of $X$, and has a trivial dependence on $t$, since (apart from contributions from the $Q$-exact term) $t$ may be reabsorbed by 
rescaling $\theta \rightarrow \theta / t$. The semi-classical limit $t \rightarrow \infty$ is therefore again exact, and the path integral localizes on the fixed points of $Q$, which are now constant maps, $d \phi^{i}=0$. After localization, the path integral then reduces to an integral over $X$.

The observables of the B-model are in one-to-one correspondence with degree $(p, q)$ polyvector fields

$$
V=V_{\bar{i}_{1} \ldots \bar{i}_{p}}^{j_{1} \ldots j_{q}} d \bar{z}^{\bar{i}_{1}} \ldots d \bar{z}^{\bar{i}_{p}} \partial_{z^{j_{1}}} \ldots \partial_{z^{j_{q}}} \in H^{p}\left(X, \Lambda^{q} T^{1,0} X\right)
$$

via $d \bar{z}^{\bar{i}} \sim \eta^{\bar{i}}, \partial_{z^{j}} \sim \theta_{j}$, since $\left\{Q, \mathcal{O}_{V}\right\}=-\mathcal{O}_{\bar{\partial} V}$. There are now two conserved ghost charges, and the anomaly in the ghost number conservation requires that

$$
\sum_{k=1}^{l} p_{k}=\sum_{k=1}^{l} q_{k}=d(1-g)
$$

For example, at genus 0 , the only vanishing correlator on a Calabi-Yau three-fold involves three $(1,1)$ polyvector fields $V_{\bar{j}}^{i}$. Using the holomorphic $(3,0)$ form, these are related to $(2,1)$ forms $\Omega_{i j l} V_{\bar{k}}^{l}$ parameterizing the complex structure of $X$. The threepoint function is

$$
\left\langle\mathcal{O}_{V_{1}} \mathcal{O}_{V_{2}} \mathcal{O}_{V_{3}}\right\rangle=\int_{X} V_{\bar{j}_{1}}^{i_{1}} V_{\bar{j}_{2}}^{i_{2}} V_{\bar{j}_{3}}^{i_{3}} \Omega_{i_{1} i_{2} i_{3}} d \bar{z}^{\bar{j}_{1}} \wedge d \bar{z}^{\bar{j}_{2}} \wedge d \bar{z}^{\bar{j}_{3}} \wedge \Omega
$$

giving access to the third derivative of the prepotential.

\subsection{Topological Strings}

Due to the conservation of the ghost number, we have seen that, from the sigma model alone, the only non-vanishing topological correlators are the three-point function on the sphere, and the vacuum amplitude on the torus. It turns out that the coupling to topological gravity allows to lift this constraint, and define arbitrary $n$-point amplitudes at any genus.

Recall that in bosonic string theory, genus $g$ amplitudes are obtained by introducing $6 g-6$ insertions of the dimension 2 ghost (or, rather, "antighost") $b$ of diffeomorphism invariance, folded with Beltrami differentials $\mu_{k} \in H^{1}\left(\Sigma, T^{1,0} \Sigma\right)$ :

$$
F_{g}=\int_{\mathcal{M}_{g}}\left\langle\prod_{k=1}^{6 g-6}\left(b, \mu_{k}\right)\right\rangle
$$

where

$$
(b, \mu)=\int_{\Sigma} d^{2} z\left[b_{z z} \mu_{\bar{z}}^{z}+b_{\bar{z} \bar{z}} \bar{\mu}_{z}^{\bar{z}}\right]
$$


This effectively produces the Weil-Peterson volume element on the moduli space $\mathcal{M}_{g}$ of complex structures on the genus $g$ Riemann surface $\Sigma$ (compactified à la DeligneMumford). Since $b$ has ghost number -1 , this exactly compensates the anomalous background charge.

After the topological twist, which identifies the BRST charge $Q$ with (say) $G_{+}$, it is natural to identify $b$ with $G_{-}$, in such a way that the energy-momentum tensor is given by $T=\{Q, b\}=\left\{G_{+}, G_{-}\right\}$. Hence, the genus $g$ vacuum topological amplitude may be written as

$$
F_{g}=\int_{\mathcal{M}_{g}}\left\langle\prod_{k=1}^{3 g-3}\left(G_{-}, \mu_{k}\right)\left(G_{ \pm}, \bar{\mu}_{k}\right)\right\rangle
$$

where the upper (resp., lower) sign corresponds to the A-model (resp., B-model). Scattering amplitudes may be obtained by inserting vertex operators with zero ghost number; these may be obtained by "descent" from a ghost number 2 operator $\mathcal{O}^{(0)}$,

$$
d \mathcal{O}^{(0)}=\left\{Q, \mathcal{O}^{(1)}\right\}, \quad d \mathcal{O}^{(1)}=\left\{Q, \mathcal{O}^{(2)}\right\}
$$

Prominent examples of $\mathcal{O}^{(0)}$ are of course $W_{i \bar{i}} \chi^{i} \chi^{\bar{i}}$ in the A-model, and $V_{j}^{\bar{i}} \eta^{\bar{i}} \theta_{j}$ in the B-model. These describes the deformations of the Kähler and complex structures, respectively. Arbitrary numbers of integrated vertex operators $\int d^{2} z \mathcal{O}^{(2)}$ can then be inserted in (4.25) without spoiling the conservation of ghost charge number.

Weighting the contributions of different genera by powers of the "topological string coupling" $\lambda$, namely

$$
F_{\text {top }}=\sum_{g=0}^{\infty} \lambda^{2 g-2} F_{g}
$$

we obtain obtain a perturbative definition of the A and B-model topological strings. Since the worldsheet is topological, the target space theory has only a finite number of fields, so is really more a field theory than a string theory. In fact, the scattering amplitudes can be reproduced by a simple action $X$, known as "holomorphic ChernSimons" in the A-model, and "Kodaira-Spencer" in the B-model; these describe the fluctuations of Kähler and complex structures, respectively. We refer the reader to [61] for an extensive discussion of these theories.

\subsection{Gromov-Witten, Gopakumar-Vafa and Donaldson-Thomas Invariants}

We now concentrate on the topological vacuum amplitude (4.27) of the A-model on a Calabi-Yau threefold $X$. Up to holomorphic anomalies that we discuss in the next section, $F_{\text {top }}$ can be viewed as a function of the complexified Kähler moduli $t^{A}=$ 
$\int_{\gamma^{A}} J$. In the large volume limit (or more generally, near a point of maximal unipotent monodromy), it has an asymptotic expansion

$$
F_{\text {top }}=-i \frac{(2 \pi)^{3}}{6 \lambda^{2}} C_{A B C} t^{A} t^{B} t^{C}-\frac{i \pi}{12} c_{2 A} t^{A}+F_{G W}
$$

where $C_{A B C}$ are the triple intersection numbers of the 4-cycles $\gamma_{A}$ dual to $\gamma^{A}$, and $c_{2 A}=\int_{\gamma_{A}} c_{2}\left(T^{(1,0)} X\right)$ are the second Chern classes of these 4-cycles. The first two terms in (4.28) are perturbative in $\alpha^{\prime}$, while $F_{G W}$ contains the effect of worldsheet instantons at arbitrary genus,

$$
F_{G W}=\sum_{g \geq 0} \sum_{\beta \in H_{2}^{+}(X)} N_{g, \beta} e^{2 \pi i \beta_{A} t^{A}} \lambda^{2 g-2}
$$

where the sum runs over effective curves with $\beta_{A} \geq 0$, and $N_{g}^{\beta}$ are (conjecturally) rational numbers known as the Gromov-Witten $(\mathrm{GW})$ invariants of $X$. It is possible to re-organize the sum in (4.29) into

$$
F_{G W}=\sum_{g \geq 0} \sum_{\beta \in H_{2}^{+}(X)} \sum_{d \geq 1} n_{g, \beta} \frac{1}{d}\left[2 \sin \left(\frac{d \lambda}{2}\right)\right]^{2 g-2} e^{2 \pi i d \beta_{A} t^{A}}
$$

The coefficients $n_{g, \beta}$ are known as the Gopakumar-Vafa (GV) invariants, and are conjectured to always be integer: indeed, one may show that the contribution of a fixed $\beta_{A}$ in (4.30) arises from the one-loop contribution of a M2-brane wrapping the isolated holomorphic curve $\beta^{A} \gamma_{A}$ in $X[62,63]$. The GV invariants can be related to the GW invariants by expanding (4.30) at small $\lambda$ and matching on to (4.29), e.g. at leading order $\lambda^{-2}$,

$$
N_{0, \beta}=\sum_{d \mid \beta_{A}} d^{3} n_{0, \beta^{A} / d}
$$

which incorporates the effect of multiple coverings for an isolated genus 0 curve.

It should be noted that the sum in (4.29) or (4.30) includes the term $\beta=0$, which corresponds to degenerate worldsheet instantons. It turns out that the only non-vanishing GV invariant at genus 0 is $n_{0,0}=-\frac{1}{2} \chi(X)$, hence

$$
F_{\text {deg }}=-\frac{1}{2} \chi(X) \sum_{d \geq 1} \frac{1}{d}\left[2 \sin \left(\frac{d \lambda}{2}\right)\right]^{2}:=-\frac{1}{2} \chi(X) f(\lambda)
$$

The function $f(\lambda)$, known as the Mac-Mahon function, may be formally manipulated into

$$
f(\lambda)=-\sum_{d \geq 1} \frac{e^{i d \lambda}}{d\left(1-e^{i d \lambda}\right)^{2}}=-\sum_{d=1}^{\infty} \sum_{n=1}^{\infty} \frac{n q^{n d}}{d}=\sum_{n=1}^{\infty} n \log \left(1-q^{n}\right)
$$


where $q=e^{i \lambda}$. The last expression converges in the upper half plane $\operatorname{Im}(\lambda)>0$, and may be taken as the definition of the Mac-Mahon function, suitable in the large coupling limit $\lambda \rightarrow i \infty$.

Exercice 13 Check that the coefficient of $q^{N}$ in the Taylor expansion of $\exp (-f)$ counts the number of three-dimensional Young tableaux with $N$ boxes.

In order to analyze its contributions at weak coupling $t=-i \lambda \rightarrow 0$, let us compute its Mellin transform ${ }^{11}$

$$
M(s)=\int_{0}^{\infty} \frac{d t}{t^{1-s}} f(t)=-\int_{0}^{\infty} \frac{d t}{t^{1-s}} \sum_{d=1}^{\infty} \sum_{n=1}^{\infty} \frac{n}{d} e^{-n d t}
$$

Exchanging the integral and sums, the result is simply expressed in terms of Euler $\Gamma$ and Riemann $\zeta$ functions,

$$
-\sum_{d=1}^{\infty} \sum_{n=1}^{\infty} \frac{n}{d}(n d)^{-s} \Gamma(s)=-\zeta(s-1) \zeta(s+1) \Gamma(s)
$$

The function $f(t)$ itself may be obtained conversely by

$$
f(t)=\frac{1}{2 \pi i} \int_{\operatorname{Re}(s)=s_{0}} M(s) t^{-s}
$$

where the contour is chosen to lie to the right of any pole of $M(s)$. Moving the contour to the left and crossing the poles generate the Laurent series expansion of $f(t)$.

To perform this computation, recall that $\Gamma(s)$ has simple poles at $s=-n, n=$ $0,1, \ldots$ with residue $(-1)^{n} / n$ !. Moreover, $\zeta(s)$ has a simple pole at $s=1$, and "trivial" zeros at $s=-2,-4,-6, \ldots$ The trivial zeros of $\zeta(s-1)$ and $\zeta(s+1)$ cancel the poles of $\Gamma(s)$ at odd negative integer, leaving only the simple poles at even strictly negative integer, a double pole at $s=0$ and a single pole at $s=2$. Altogether, returning to the variable $\lambda=i t$,we obtain the Laurent series expansion

$$
f(\lambda)=\frac{\zeta(3)}{\lambda^{2}}+\frac{1}{12} \log (i \lambda)-\zeta^{\prime}(1)+\sum_{g=2}^{\infty} \frac{B_{2 g} B_{2 g-2} \lambda^{2 g-2}}{(2 g-2) !(2 g-2)(2 g)}
$$

where we further used the relation $\zeta(3-2 g)=-B_{2 g-2} /(2 g-2)(g \geq 2)$ between the values of $\zeta$ and Bernoulli numbers.

\footnotetext{
${ }^{11}$ The following argument is due to S. Miller (private communication), and considerably simplifies the computation in [64].
} 
The leading term, proportional to $\zeta(3)$, leads to a constant shift $-1 / 2 \chi(X) \zeta(3)$ in the tree-level prepotential, and can be traced back to the tree-level $R^{4}$ term in the 10-dimensional effective action, reduced along $X[65,66,67]$. The terms with $g \geq 2$ were first computed using heterotic/type II duality [68], and impressively agree with an independent computation of the integral over the moduli space $\mathcal{M}_{g}$ [69],

$$
\int_{\mathcal{M}_{g}} c_{g-1}^{3}=-\frac{B_{2 g} B_{2 g-2} \lambda^{2 g-2}}{(2 g-2) !(2 g-2)(2 g)}
$$

The logarithmic correction in (4.37) originates from the double pole of $M(s)$ at $s=0$, and has no simple interpretation yet. It is nevertheless forced if one accepts that the correct non-perturbative completion of the degenerate instanton series is the MacMahon function [70, 64].

For completeness, let us finally mention the relation to a third type of topological invariants, known as Donaldson-Thomas invariants $n_{D T}\left(q_{A}, m\right)$ [71]: these count "ideal sheaves" on $X$, which can be understood physically as bound states of $m$ D0-branes, $q_{A}$ D2-branes wrapped on $q^{A} \gamma_{A} \in H_{2}(\mathbb{Z})$ and a single D6-brane. S-duality implies $[72,73]$ that the partition function of Donaldson-Thomas invariants is related to the partition function of Gromov-Witten invariants by $[74,75]$

$$
\sum_{q^{A} \in H_{2}(\mathbb{Z}), m \in \mathbb{Z}} n_{D T}\left(q_{A}, m\right) e^{i t_{A} q^{A}} q^{m}=\exp \left[F_{G W}(t, \lambda)-\frac{\chi}{2} f(\lambda)\right]
$$

where $q=-e^{i \lambda}$. Such a relation may be understood from the fact that a curve may be represented either by a set of a equations (the Donaldson-Thomas side), or by an explicit parameterization (the Gromov-Witten side). This conjecture has been recently proven for any toric three-fold $X[76]$.

\subsection{Holomorphic Anomalies and the Wave Function Property}

In the previous subsection, we assumed that the topological amplitude was a function of the holomorphic moduli $t_{i}$ only. This is naively warranted by the fact that the variation of the anti-holomorphic moduli $\overline{t^{i}}$ results in the insertion of an (integrated) $Q$-exact operator, $\phi_{\bar{i}}=\left\{G^{+},\left[\bar{G}^{+}, \bar{\phi}_{\bar{i}}\right]\right\}$. By the same naive reasoning, one would expect that the

$n$-point functions $C_{i_{1} \ldots i_{n}}^{(g)}$ be independent of $\bar{t}$, and equal to the $n$-th order derivative of the vacuum amplitude $F_{g}$ with respect to $t^{i_{1}}, \ldots t^{i_{n}}$. Both of these expectations turn out to be wrong, due to boundary contributions in the integral over the moduli space of genus $g$ Riemann surfaces. By analyzing these contributions carefully, Berschadsky, Cecotti, Ooguri and Vafa [61] (BCOV) have shown that the $\bar{t}^{i}$ derivative of $F_{g}$ is related 
to $F_{h<g}$ at lower genera via ${ }^{12}$

$$
\bar{\partial}_{\bar{i}} F_{g}=\frac{1}{2} e^{2 K} \bar{C}_{\overline{i j} \bar{k}} g^{j \bar{j}} g^{k \bar{k}}\left(D_{j} D_{k} F_{g-1}+\sum_{h=1}^{g-1}\left(D_{j} F_{h}\right)\left(D_{k} F_{g-h}\right)\right)
$$

where $D_{i} F_{g}=\left(\partial_{i}-(2-2 g) \partial_{i} K\right) F_{g}$, as appropriate for a section of $\mathcal{L}^{2-2 g}$. In (4.40), the first term on the r.h.s. originates from the boundary of $\mathcal{M}_{g}$ where one non-contractible handle of $\Sigma$ is pinched, whereas the second term corresponds to the limit where a homologically trivial cycle vanishes, disconnecting $\Sigma$ into two Riemann surfaces with genus $h$ and $g-h$. A similar identity can be derived for $n$-point functions. Moreover, the latter are indeed obtained from the vacuum amplitude by derivation with respect to $t^{i}$, provided one uses a covariant derivative taking into account the Levi-Civita and Kähler connections:

$$
C_{i_{1} \ldots i_{n}}^{(g)}=\left\{\begin{array}{cl}
D_{i_{1}} \ldots D_{i_{n}} F_{g} & \text { for } g \geq 1, n \geq 1 \\
D_{i_{1}} \ldots D_{i_{n-3}} C_{i_{n-1} i_{n-1} i_{n}} & \text { for } g=0, n \geq 3 \\
0 & \text { for } 2 g-2+n \leq 0
\end{array}\right.
$$

where $C_{i j k}$ is the tree-level three-point function. The resulting identities may be summarized by defining the "topological wave-function"

$$
\Psi_{\mathrm{BCOV}}=\lambda^{\frac{\chi}{24}-1} \exp \left[\sum_{g=0}^{\infty} \sum_{n=0}^{\infty} \frac{1}{n !} \lambda^{2 g-2} C_{i_{1} \ldots i_{n}}^{(g)} x^{i_{1}} \ldots x^{i_{n}}\right]
$$

Note that $\Psi_{\mathrm{BCOV}}$ does not incorporate the genus 1 vacuum amplitude. In terms of this object, the identities (4.40) (or rather their generalization to $n$-point functions) and (4.41) are summarized by the two equations

$$
\begin{aligned}
& \partial_{\bar{t}^{i}}=\frac{\lambda^{2}}{2} e^{2 K} \bar{C}_{\overline{i j} \bar{k} g^{j j}} g^{k \bar{k}} \frac{\partial^{2}}{\partial x^{j} \partial x^{k}}-g_{\overline{i j}} x^{j}\left(\lambda \frac{\partial}{\partial \lambda}+x^{k} \frac{\partial}{\partial x^{k}}\right) \\
& \partial_{t^{i}}=\Gamma_{i j}^{k} x^{j} \frac{\partial}{\partial x^{k}}-\partial_{i} K\left(\frac{\chi}{24}-1-\lambda \frac{\partial}{\partial \lambda}\right)+\frac{\partial}{\partial x^{i}}-\partial_{i} F_{1}-\frac{1}{2 \lambda^{2}} C_{i j k} x^{j} x^{k}
\end{aligned}
$$

By rescaling $x^{i} \rightarrow \lambda x^{i}, \Psi \rightarrow e^{f_{1}(t)} \Psi$ where $f_{1}(t)$ is the holomorphic function in the general solution

$$
F_{1}=-\frac{1}{2} \log |g|+\left(\frac{n_{V}+1}{2}-\frac{\chi}{24}+1\right) K+f_{1}(t)+\bar{f}_{1}(\bar{t})
$$

\footnotetext{
${ }^{12}$ When $g=1$, the holomorphic equation becomes second order, and can be read off from $(4.43)$ below.
} 
of the holomorphic anomaly equation for $F_{1}$, E. Verlinde [77] was able to recast $(4.43),(4.44)$ in a form involving only special geometry data,

$$
\begin{aligned}
\partial_{\bar{t}^{i}} & =\frac{1}{2} e^{2 K} \bar{C}_{\overline{i j} \bar{k}} g^{j \bar{j}} g^{k \bar{k}} \frac{\partial^{2}}{\partial x^{j} \partial x^{k}}+g_{\overline{i j}} x^{j} \frac{\partial}{\partial \lambda^{-1}} \\
\nabla_{i}-\Gamma_{i j}^{k} x^{j} \frac{\partial}{\partial x^{k}} & =\frac{1}{2} \partial_{t^{i}} \log |g|+\frac{1}{\lambda} \frac{\partial}{\partial x^{i}}-\frac{1}{2} e^{-2 K} C_{i j k} x^{j} x^{k}
\end{aligned}
$$

where

$$
\nabla_{i}=\partial_{i}+\partial_{i} K\left(x^{k} \frac{\partial}{\partial x^{k}}-\lambda \frac{\partial}{\partial \lambda}+\frac{n_{V}+1}{2}\right)
$$

Here, $|g|=\operatorname{det}\left(g_{i j}\right)$. The implications of these equations were understood in [78] and further clarified in $[77,79]: \Psi(t, \bar{t} ; x, \lambda)$ should be thought of as a single state $|\Psi\rangle$ in a Hilbert space, expressed on a $(t, \bar{t})$-dependent basis of coherent states,

$$
\Psi(t, \bar{t} ; x, \lambda)={ }_{(t, \bar{t})}\left\langle x^{i}, \lambda \mid \Psi\right\rangle
$$

This is most easily explained in the B-model, where $\left(x, \lambda^{-1}\right)$ and their complex conjugate can be viewed as the coordinates of a 3 -form $\gamma \in H^{3}(X, \mathbb{R})$ on the Hodge decomposition

$$
\gamma=\lambda^{-1} \Omega+x^{i} D_{i} \Omega+x^{\bar{i}} D_{\bar{i}} \bar{\Omega}+\bar{\lambda}^{-1} \bar{\Omega}
$$

The space $H^{3}(X, \mathbb{R})$ admits a symplectic structure

$$
\omega=i e^{-K}\left(g_{i \bar{j}} d x^{i} \wedge d \bar{x}^{\bar{j}}-d \lambda^{-1} \wedge d \bar{\lambda}^{-1}\right)
$$

inherited from the anti-symmetric pairing $(\alpha, \beta)=\int_{X} \alpha \wedge \beta$, which leads to the Poisson brackets between the coordinates

$$
\left\{\lambda^{-1}, \bar{\lambda}^{-1}\right\}=i e^{K}, \quad\left\{x^{i}, \bar{x}^{\bar{j}}\right\}=-i g^{i \bar{j}}
$$

The phase $H^{3}(X, \mathbb{R})$ may be quantized by considering functions (or rather half-densities, to account for the zero-point energy) of $\left(\lambda^{-1}, x^{i}\right)$ and representing $\bar{\lambda}^{-1}$ and $\bar{x}^{\bar{i}}$ as derivative operators,

$$
\bar{\lambda}^{-1}=-e^{K} \frac{\partial}{\partial \lambda^{-1}}, \quad \bar{x}^{\bar{i}}=e^{K} g^{\overline{i j}} \frac{\partial}{\partial x^{j}}
$$

The resulting wave function $\Psi(t, \bar{t} ; \lambda, x)$ carries a dependence on the "background" variables $(t, \bar{t})$ since the decomposition (4.50) does depend on these variables via $\Omega$. A variation of $t$ and $\bar{t}$ generically mixes $\left(\lambda^{-1}, x\right)$ with their canonical conjugate, and so may be compensated by an infinitesimal Bogolioubov transformation, reflected in 
(4.46),(4.47) do. In fact, we can check that these two equations are hermitean conjugate under the inner product

$$
\begin{aligned}
\left\langle\Psi^{\prime} \mid \Psi\right\rangle= & \int d x^{i} d \bar{x}^{\bar{i}} d \lambda^{-1} d \bar{\lambda}^{-1}|g| e^{-\frac{n_{V}+1}{2} K} \\
& \exp \left(-e^{-K} x^{i} g_{i \bar{j}} \bar{x}^{j}+e^{-K} \lambda^{-1} \bar{\lambda}^{-1}\right) \Psi^{\prime *}(t, \bar{t} ; \bar{x}, \bar{\lambda}) \Psi(t, \bar{t} ; x, \lambda)
\end{aligned}
$$

which is the natural inner product arising in Kähler quantization. In contrast to $\Psi$ and $\Psi^{\prime}$ separately, the inner product is background independent (and, in fact, a pure number), by virtue of the anomaly equations.

Exercice 14 Show that in the harmonic oscillator Hilbert space, the wave functions in the real and oscillator polarizations are related by (abusing notation)

$$
f(q)=\int d a^{\dagger} e^{i a^{\dagger} q \sqrt{2}+q^{2} / 2-\left(a^{\dagger}\right)^{2} / 2} f\left(a^{\dagger}\right)=\int d a e^{-i a q \sqrt{2}-q^{2} / 2+a^{2} / 2} f(a)
$$

Conclude that the inner product in oscillator basis is given by

$$
\int d q f^{*}(q) g(q)=\int d a d a^{\dagger} e^{-a a^{\dagger}} f^{*}(a) g\left(a^{\dagger}\right)
$$

This indicates that there exists a different polarization without any background dependence: choose a real symplectic basis $\gamma^{I}, \gamma_{I}$ of three-cycles in $H_{3}(X, \mathbb{Z})$, and expand

$$
\gamma=p^{I} \gamma_{I}+q_{I} \gamma^{I}
$$

The symplectic form is now just $\omega=d q_{I} \wedge d p^{I}$, so $H_{3}(X, \mathbb{R})$ can be quantized by considering functions of $p^{I}$, and representing $q_{I}$ as $i \partial / \partial p^{I}$; equivalently, one may introduce a set of coherent states $\left|p^{I}\right\rangle$, and define the wave function in the "real" polarization, $\Psi_{\mathbb{R}}\left(p^{I}\right)=\left\langle p^{I} \mid \Psi\right\rangle$. This is related to the wave function in the Kähler polarization by a finite Bogolioubov transformation ${ }^{13}$

$$
\Psi_{\mathrm{BCOV}}(t, \bar{t} ; \lambda, x)=\int d p^{I} \Psi(p ; t, \bar{t} ; \lambda, x) \Psi_{\mathbb{R}}\left(p^{I}\right)
$$

where the intertwiner $\Psi(p ; t, \bar{t} ; \lambda, x)$ is a general solution of $(4.46),(4.47)$, equal to the overlap of the coherent states $\langle x, \lambda \mid p\rangle[77,79]$

$$
\begin{aligned}
\Psi(p ; t, \bar{t} ; \lambda, x) & =e^{-\left(n_{V}+1\right) K / 2} \sqrt{\operatorname{det} g_{i j}} \exp \left[-\frac{1}{2} p^{I} \bar{\tau}_{I J} p^{J}+2 i p^{I}[\operatorname{Im} \tau]_{I J}\left(\lambda^{-1} X^{I}+e^{-K / 2} x^{i} f_{i}^{I}\right)\right. \\
& \left.+i\left(\lambda^{-2} X^{I}[\operatorname{Im} \tau]_{I J} X^{J}+2 \lambda^{-1} e^{-K / 2} x^{i} f_{i}^{I}[\operatorname{Im} \tau]_{I J} X^{J}+e^{-K} x^{i} f_{i}^{I}[\operatorname{Im} \tau]_{I J} f_{j}^{J} x^{j}\right)\right]
\end{aligned}
$$

\footnotetext{
${ }^{13} \mathrm{~A}$ precursor of this formula was already found in BCOV, although not recognized as such.
} 
While the topological wave function in the real polarization has the great merit of being background independent, it is nevertheless not canonical, since it depends on a choice of symplectic basis. As usual in quantum mechanics, changes of symplectic basis correspond are implemented by the metaplectic representation of $S p\left(2 n_{V}+2\right)$ (or rather, of its metaplectic cover). In particular, upon exchanging $A$ and $B$ cycles, $\Psi_{\mathbb{R}}\left(p^{I}\right)$ is turned into its Fourier transform, which is the quantum analogue of the classical property discussed in Exercice 4 on page 14.

For completeness, let us mention that there exists a different "holomorphic" polarization, intermediate between the Kähler and real polarizations, where the topological amplitude is a purely holomorphic function of the background moduli $t^{i}$, satisfying a heat-type equation analogous to the Jacobi theta series [79]. We shall return to this in Section (7.5).

\section{Higher Derivative Corrections and Topological Strings}

In this section, we return to the realm of physical string theory, and explain how a special class of higher-derivative terms in the low-energy effective action can be reduced to a topological string computation. We then discuss how these terms affect the Bekenstein-Hawking entropy of black holes, and formulate the Ooguri-StromingerVafa conjecture, which purportedly relates the topological amplitude to the microscopic degeneracies.

\subsection{Gravitational F-terms and Topological Strings}

In general, higher-derivative and higher-genus corrections in string theory are very hard to compute: the integration measure on supermoduli space is ill-understood beyond genus 2 (see [80] for the state of the art at genus 2), and the current computation schemes (with the exception of the pure spinor superstring, see e.g. [81]) are nonmanifestly supersymmetric, requiring to evaluate many different scattering amplitudes at a given order in momenta.

Fortunately, $\mathcal{N}=2$ supergravity coupled to vector multiplets has an off-shell superspace description, which greatly reduces the number of diagrams to be computed, and also provides a special family of "F-term" interactions, which can be efficiently computed. The most convenient formulation starts from $\mathcal{N}=2$ conformal supergravity and fixes the conformal gauge so as to reduce to Poincaré supergravity (see [35] for an extensive review of this approach). The basic objects are the Weyl and matter 
chiral superfields,

$$
\begin{array}{r}
W_{\mu \nu}(x, \theta)=T_{\mu \nu}-\frac{1}{2} R_{\mu \nu \rho \sigma} \epsilon_{\alpha \beta} \theta^{\alpha} \sigma_{\lambda \rho} \theta^{\beta}+\ldots \\
\Phi^{I}(x, \theta)=X^{I}+\frac{1}{2} \mathcal{F}_{\mu \nu}^{I} \epsilon_{\alpha \beta} \theta^{\alpha} \sigma^{\mu \nu} \theta^{\beta}+\ldots
\end{array}
$$

where $\alpha, \beta=1,2 . T_{\mu \nu}$ is an auxiliary anti-selfdual tensor, identified by the (tree-level) equations of motion as the graviphoton (3.14). From $W$ one may construct the scalar chiral superfield

$$
W^{2}(x, \theta)=T_{\mu \nu} T^{\mu \nu}-2 \epsilon_{i j} \theta^{i} \sigma^{\mu \nu} \theta^{j} R_{\mu \nu \lambda \rho} T^{\lambda \rho}-\left(\theta^{i}\right)^{2}\left(\theta^{j}\right)^{2} R_{\mu \nu \lambda \rho} R^{\mu \nu \lambda \rho}+\ldots
$$

where the anti-self dual parts of $R$ and $T$ are understood. Starting with any holomorphic, homogeneous of degree two function $F\left(\Phi^{I}, W^{2}\right)$, regular at $W^{2}=0$,

$$
F\left(\Phi^{I}, W^{2}\right):=\sum_{g=0}^{\infty} F_{g}\left(\Phi^{I}\right) W^{2 g}
$$

(where $F_{g}$ is homogeneous of degree $2-2 g$ ) one may construct the chiral integral

$$
\int d^{4} \theta d^{4} x F\left(\Phi, W^{2}\right)=S_{\text {tree }}+\int \sum_{g=1}^{\infty} F_{g}\left(X^{I}\right)\left(g R^{2} T^{2 g-2}+2 g(g-1)(R T)^{2} T^{2 g-4}\right)+\ldots
$$

which reproduces the tree-level $\mathcal{N}=2$ supergravity action based on the prepotential $F_{0}$, plus an infinite sum of higher-derivative "F-term" gravitational interactions (plus non-displayed terms). $F\left(\Phi^{I}, W^{2}\right)$ is known as the generalized prepotential.

In order to compute the coefficients $F_{g}\left(X^{I}\right)$, one should compute the scattering amplitude of 2 gravitons and $2 g-2$ graviphotons in type II (A or B) string theory at leading order in momenta compactified on a Calabi-Yau threefold $X$. This problem was studied in [82], where it was shown (as anticipated in [61]) that it reduces to a computation in topological string theory. We now briefly review the argument.

The graviphoton originates from the Ramond-Ramond sector; taking into account the peculiar couplings of RR states to the dilaton, $F_{g}$ is identified as a genus $g$ amplitude $^{14}$. Perturbative contributions from a different loop order or non-perturbative ones are forbidden, since the type II dilaton is an hypermultiplet. The graviton vertex operator (in the 0 superghost picture) is

$$
V_{g}^{(0)}=h_{\mu \nu}\left(\partial X^{\mu}+i p \cdot \psi \psi^{\mu}\right)\left(\bar{\partial} X^{\mu}+i p \cdot \tilde{\psi} \tilde{\psi}^{\mu}\right) e^{i p X}
$$

\footnotetext{
${ }^{14}$ When $X$ is K3-fibered, and in the limit of a large base, one can obtain the generalized prepotential from a one-loop heterotic computation [83, 68].
} 
The vertex operator of the graviphoton (in the $-1 / 2$ superghost picture) is

$$
V_{T}^{(-1 / 2)}=\epsilon_{\mu} p_{\nu} e^{-(\phi+\tilde{\phi}) / 2}\left(S \sigma_{\mu \nu} \tilde{S} \Sigma_{+} \tilde{\Sigma}_{\mp}+c c\right) e^{i p X}
$$

where $S, \tilde{S}$ are spin fields in the 4 non-compact dimensions, and $\Sigma_{ \pm}$is the spectral flow operator (4.7) in the $N=(2,2)$ SCFT. The insertion of $2 g-2$ graviphotons induces a background charge $\int \frac{\sqrt{3}}{2} H R^{(2)}$, which induces the topological twist $L_{0} \rightarrow L_{0}-\frac{1}{2} J$. The same process takes place in the SCFT describing the 4 non-compact directions. As a result, the bosonic and fermionic fluctuation determinants cancel. Moreover, choosing the polarizations of the graviton and graviphotons to be anti-self-dual, only the $\psi \psi \tilde{\psi} \tilde{\psi}$ terms in (5.6) contribute after summing over spin structures, and cancel against the contractions of the spin fields $S \tilde{S}$.

Now we turn to the cancellation of the superghost charge: the integration over supermoduli brings down $2 g-2$ powers of the picture-changing operator $e^{\phi} T_{F} \times c c$, where $T_{F}=G_{+}+G_{-}$is the supercurrent. In order to cancel the superghost background charge $2 g-2$, it is therefore necessary to transform $g-1$ of the $2 g-2$ graviphoton vertex operators in the $+1 / 2$ picture. In total, we thus have $3 g-3$ insertions of $T_{F}$. By conservation of the $U(1)$ charge, it turns out that only the $G_{-}$and $\tilde{G}_{ \pm}$parts of $T_{F}$ and $\tilde{T}_{F}$ contribute. Finally, we reach

$$
A_{g}=(g !)^{2} \int_{\mathcal{M}_{g}}\left\langle\prod_{a=1}^{3 g-3}\left(\mu_{a} G_{-}\right)\left(\tilde{\mu}_{a} \tilde{G}_{ \pm}\right)\right\rangle=(g !)^{2} F_{g}
$$

where the upper (lower) sign corresponds to type IIB (resp. IIA). We conclude that the generalized prepotential $F_{g}(X)$ in type IIA (B) string theory compactified on $X$ is equal to the all genus vacuum amplitude (4.27) of the A (resp. B)-model topological string. The precise identification of the variables is

$$
F_{\text {top }}=\frac{i \pi}{2} F_{S U G R A}, \quad t^{A}=\frac{X^{A}}{X^{0}}, \quad \lambda=\frac{\pi}{4} \frac{W}{X^{0}}
$$

To be more precise, the vacuum topological amplitude $F_{g}(t, \bar{t})$, computes the physical $R^{2} T^{2 g-2}$ coupling; it differs from the the holomorphic "Wilsonian" coupling $F_{g}(X)$ appearing in (5.5) due to the contributions of massless particles. It is often assumed that these contributions are removed by taking $\bar{t} \rightarrow \infty$ keeping $t$ fixed; it would be interesting to determine whether this is indeed equivalent to going to using the real polarized topological wave function (4.58).

For completeness and later reference, let us mention that, by a similar reasoning, the topological B-model (resp. A) in type IIA (resp. B) computes higher-derivative 
interactions between the hypermultiplets, of the form [82]

$$
\tilde{S}=\int d^{4} x \sum_{g=1}^{\infty} \tilde{F}_{g}(X)\left[g(\partial \partial S)^{2}(\partial Z)^{2 g-2}+2 g(g-1)(\partial \partial S \partial Z)^{2}(\partial Z)^{2 g-4}\right]
$$

where $(S, Z)$ describes the universal hypermultiplet. It is also an interesting open problem to construct an off-shell superfield formalism which would describe all these interactions at once as F-terms.

\subsection{Bekenstein-Hawking-Wald Entropy}

In general, higher-derivative corrections affect the macroscopic entropy of black holes in two ways:

i) they affect the actual solution, and in particular the relation between the horizon geometry and the data measured at infinity;

ii) by modifying the stress-energy tensor, they change the relation between geometry and entropy.

Moreover, since subleading contributions to the statistical entropy are non-universal, comparison with the microscopic result requires

iii) specifying the statistical ensemble implicit in the low-energy field theory.

As far as i) is concerned, and provided that we restrict to BPS black holes, the fact that the generalized $\mathcal{N}=2$ supergravity has an off-shell description simplifies the computation drastically: the supersymmetry transformation rules are the same as at tree-level; Cardoso, de Wit and Mohaupt [84, 85, 86, 87] (CdWM) have shown that the horizon geometry is still $A d S_{2} \times S^{2}$, while the value of the moduli is governed by the a generalization of the stabilisation equations (3.42),

$$
\operatorname{Re}\left(Y^{I}\right)=p^{I}, \quad \operatorname{Re}\left(G_{I}\right)=q_{I}, \quad W^{2}=2^{8}
$$

where $G_{I}$ is now the derivative of the generalized prepotential, $G_{I}=\partial F\left(Y, W^{2}\right) / \partial Y^{I}$.

As far as ii) is concerned, Wald [88] has given a general prescription for obtaining an entropy functional that satisfies the first law ${ }^{15}$ of thermodynamics, in the context of a Lagrangian $\mathcal{L}(R)$ with a general dependence on the Riemann tensor:

$$
S_{B H W}=2 \pi \int_{\Sigma} \frac{\partial \mathcal{L}}{\partial R_{\mu \nu \rho \sigma}} \epsilon^{\mu \nu} \epsilon^{\rho \sigma} \sqrt{h} d \Omega
$$

where $h$ is the induced metric on the horizon $\Sigma$, and $\epsilon^{\mu \nu}$ is the binormal.

\footnotetext{
${ }^{15}$ Whether or not it satisfies the second law seems to remain an open problem.
} 
Exercice 15 Show that for $\mathcal{L}=-\frac{1}{16 \pi G} R$, (5.12) reduces to the usual BekensteinHawking area law.

While the $\mathcal{N}=2$ corrected Lagrangian does not have such a simple form, CdWM adapted Wald's construction and found a simple result generalizing (3.43)

$$
S_{B H W}=\frac{i \pi}{4}\left(\bar{Y}^{I} G_{I}-Y^{I} \bar{G}_{I}\right)-\frac{\pi}{2} \operatorname{Im}\left[W \partial_{W} F\right]
$$

where the r.h.s. should be evaluated at the attractor point (5.11).

It should be emphasized that this result takes into account the contributions of the F-terms only; at a given order in momenta, there surely are other "D-terms" interactions which would contribute to the thermodynamical entropy. The results below suggest that such contributions should cancel for BPS black holes: a beautiful proof has been given in [89], but assumes that the black hole can be lifted to 5 dimensions.

\subsection{The Ooguri-Strominger-Vafa Conjecture}

As noticed in [90], using the homogeneity relation $Y^{I} G_{I}+W \partial_{W} F=2 F$, it is possible to perform the same manipulation as in (3.45) to rewrite the entropy (5.13) as a Legendre transform

$$
\begin{aligned}
S_{B H W} & =\frac{i \pi}{4}\left[\left(Y^{I}-2 i \phi^{I}\right) G_{I}-\left(\bar{Y}^{I}+2 i \phi^{I}\right) \bar{G}_{I}\right]+\frac{i \pi}{4}\left[W \partial_{W} F-\bar{W} \partial_{\bar{W}} \bar{F}\right] \\
& =\frac{i \pi}{2}(F-\bar{F})+\frac{\pi}{2} \phi^{I}\left(G_{I}+\bar{G}_{I}\right) \\
& =\mathcal{F}\left(p^{I}, \phi^{I}\right)+\pi \phi^{I} q_{I}
\end{aligned}
$$

of the "topological free energy" $\mathcal{F}\left(p^{I}, \phi^{I}\right)$, which now incorporates the infinite series of higher derivative F-term corrections,

$$
\mathcal{F}\left(p^{I}, \phi^{I}\right)=-\pi \operatorname{Im}\left[F\left(Y^{I}=p^{I}+i \phi^{I} ; W^{2}=2^{8}\right)\right]
$$

In fact, there are now general arguments [91, 89] to the effect that the BekensteinHawking-Wald entropy is equal the Legendre transform of the Lagrangian evaluated on the near-horizon geometry; in the case of $\mathcal{N}=2$ supergravity, the equality of this Lagrangian with the topological free energy $\mathcal{F}(p, \phi)$ was checked recently in [13].

As argued by OSV, the simplicity of (5.16) strongly suggests that the thermodynamical ensemble implicit in the BHW entropy is a "mixed" ensemble, where magnetic charges are treated micro-canonically but electric charges are treated canonically; the thermodynamical relation (5.16) should then perhaps be viewed as an approximation of an exact relation between two different statistical ensembles

$$
\sum_{q_{I} \in \Lambda_{e l}} \Omega\left(p^{I}, q_{I}\right) e^{-\pi \phi^{I} q_{I}} \stackrel{?}{=} e^{\mathcal{F}\left(p^{I}, \phi^{I}\right)}
$$


where $\Omega\left(p^{I}, q_{I}\right)$ are the "microcanonical" degeneracies of states with fixed charges $\left(p^{I}, q_{I}\right)$, and the sum runs over the lattice $\Lambda_{e l}$ of electric charges. Making use of (5.17), the right-hand side may be rewritten as

$$
\sum_{q_{I} \in \Lambda_{e l}} \Omega\left(p^{I}, q_{I}\right) e^{-\pi \phi^{I} q_{I}} \stackrel{?}{=}\left|\Psi_{\mathrm{top}}\left(p^{I}+i \phi^{I}, 2^{8}\right)\right|^{2}
$$

or, conversely,

$$
\Omega\left(p^{I}, q_{I}\right) \stackrel{?}{=} \int d \phi^{I}\left|\Psi_{\mathrm{top}}\left(p^{I}+i \phi^{I}, 2^{8}\right)\right|^{2} e^{\pi \phi^{I} q_{I}}
$$

It should be stressed that going from the "OSV fact" (5.20) to the OSV conjecture (5.19) involves a considerable leap of faith which should not be taken lightly.

In its strongest form, the conjecture provides a way to compute the exact microscopic degeneracies $\Omega\left(p^{I}, q_{I}\right)$ from the topological string amplitude $F\left(X, W^{2}\right)$. However, this would most likely require extending the definition of $F\left(X, W^{2}\right)$ to include non-perturbative contributions in $W$. Conversely, one may hope to understand the non-perturbative completion of the topological string from a detailed knowledge of black hole degeneracies. The weaker, more concrete form of the OSV conjecture states that the relation (5.20) should hold asymptotically to all orders in inverse charges.

The conjecture calls for some immediate remarks:

- While the formula (5.20) at first sight seems to treat electric and magnetic charges differently, it is nevertheless invariant under electric-magnetic duality, provided that the topological amplitude $\Psi_{\text {top }}$ transforms in the metaplectic representation of the symplectic group (see Exercice 17 on page 56 below). Thus, $\Psi_{\text {top }}$ should be understood as the topological wave function $\Psi_{\mathbb{R}}\left(p^{I}\right)$ in the real polarization [77] (which may be different from the $\bar{t} \rightarrow \infty$ limit, as stressed below (5.9)).

- Upon analytically continuing $\phi^{I}=i \chi^{I}$, the r.h.s. of (5.20) defines the Wigner function associated to the quantum state $\Psi_{\text {top }}$ (we shall return to this observation in Section 7). As it is well known in quantum mechanics, it is not definite positive, so if the strong conjecture is to hold, $\Omega(p, q)$ should probably refer to an index rather than to an absolute degeneracy of states. This fits well with the fact that $\Psi_{\text {top }}$ contains only information about F-term interactions, which is probably insufficient to encode the absolute degeneracies.

- Due to charge quantization, the l.h.s. of (5.19) is formally periodic under imaginary shifts $\phi^{I} \rightarrow \phi^{I}+2 i k^{I}, k^{I} \in \mathbb{Z}$, which is not the case of the r.h.s. $\left|\Psi_{\text {top }}\right|^{2}$. This can be repaired by replacing $(5.19)$ by

$$
\sum_{q_{I} \in \Lambda_{e l}} \Omega\left(p^{I}, q_{I}\right) e^{-\pi \phi^{I} q_{I}} \stackrel{?}{=} \sum_{k^{I} \in \Lambda_{e l}^{*}} \Psi^{*}\left(p^{I}-2 k^{I}-i \phi^{I}\right) \Psi\left(p^{I}+2 k^{I}+i \phi^{I}\right)
$$


without affecting the converse statement (5.20). This r.h.s. of this equation is reminiscent of a theta series. Similar averaging have indeed been found to occur in some non-compact models $[92,93]$. Note however that this averaging renders the prospect of recovering the non-perturbative generalization of $\Psi_{\top}$ from $\Omega\left(p^{I}, q_{I}\right)$ more uncertain.

- The sum on the 1.h.s. of (5.19) does not appear to converge, which reflects the thermodynamical instability of the mixed ensemble. Moreover, specifying the integration contour in (5.20) would require understanding the singularities of the topological amplitude. These subtleties do not affect the weak form of the conjecture, since the saddle point approximation to (5.20) is independent of the details of the contour.

- A variant of the OSV conjecture (5.20) has been proposed in [42], which involves an integral over both $X^{I}$ and $\bar{X}^{I}$, or equivalently a thermodynamical ensemble with fixed electric and magnetic potentials (see Exercice 7 on page 21). It would be interesting to demonstrate the equivalence of this approach based on the holomorphic polarization of the topological amplitude [77].

The OSV conjecture has been successfully tested in the case of non-compact CalabiYau manifolds of the form $O(-m) \oplus O(2 g-2+m) \rightarrow \Sigma_{g}$, where $\Sigma$ is a genus $g$ Riemann surface [92, 93]: BPS states are counted by topologically twisted SYM on $N$ D4-brane wrapped on a 4-cycle $O(-m) \rightarrow \Sigma$, which is equivalent to 2D Yang Mills (or a $q$ deformation thereof, when $g \neq 1$ ). At large $N$, the partition function of 2D Yang-Mills indeed factorizes into two chiral halves [94], which indeed agree with the topological amplitude computed independently. Exponentially suppressed corrections to the large $N$ limit of 2D Yang-Mills have been studied in [95], and seem to call for a "second quantization" of the r.h.s. of (5.19). For $\mathcal{N}=4$ and $\mathcal{N}=8$ compactifications on $K 3 \times T^{2}$ and $T^{6}$, the formula (5.20) has been compared to the prediction for dyons degeneracies based on U-dualities, and agreement has been found in the semi-classical approximation [96]. More recently, several "derivations" of the weak form of the OSV conjecture have been given, using a $M 2-\bar{M} 2$ or $D 6-\bar{D} 6$ representation of the black hole, and some modular properties of the partition function [97, 98].

In the next Section, we shall present an extensive test of the OSV conjecture in the context of small black holes in $\mathcal{N}=4$ and $\mathcal{N}=2$ theories, whose microscopic counting can be made exactly. 


\section{Precision Counting of Small Black Holes}

In order to test the OSV conjecture, one should be able to compute subleading corrections to the microscopic degeneracies $\Omega(p, q)$. Due to subtleties in the "black string" CFT description of 4-dimensional black holes, it has not been hitherto possible to reliably compute subleading corrections to (2.17) for generic BPS black holes.

On the other hand, the heterotic string has a variety of BPS excitations which can be counted exactly using standard wordsheet techniques. Since these states are only charged electrically (in the natural heterotic polarization), their Bekenstein-Hawking entropy evaluated using tree-level supergravity vanishes. This means that higherderivative corrections cannot be neglected, and indeed, upon including $R^{2}$ corrections to the effective action, a smooth horizon with finite area is obtained. We refer to these states as "small black holes", to be contrasted with "large black holes" which have non-vanishing entropy already at tree level. Our presentation follows [99, 70, 64].

\subsection{Degeneracies of DH states and the Rademacher formula}

The simplest example to study this phenomenon is the heterotic string compactified on $T^{6}$. A class of perturbative BPS states, known as "Dabholkar-Harvey" (DH) states, can be constructed by tensoring the ground state of the right-moving superconformal theory with a level $N$ excitation of the 24 left-moving bosons, and adding momentum $n$ and winding $w$ along one circle in $T^{6}$ such that the level matching condition $N-1=n w$ is satisfied [100, 101]. The number of distinct DH states with fixed charges $(n, w)$ is $\Omega(n, w)=p_{24}(N)$, where $p_{24}(N)$ is the number of partitions on $N$ into the sum of 24 integers (up to an overall factor of 16 corresponding to the size of short $\mathcal{N}=4$ multiplets, which we will always drop). Accordingly, the generating function of the degeneracies of DH states is

$$
\sum_{N=0}^{\infty} p_{24}(N) q^{N-1}=\frac{1}{\Delta(q)},
$$

where $\Delta(q)$ is Jacobi's discriminant function

$$
\Delta(q)=\eta^{24}(q)=q \prod_{n=1}^{\infty}\left(1-q^{n}\right)^{24}
$$

In order to determine the asymptotic density of states at large $N-1=n w$, it is convenient to extract $d(N)$ from the partition function (6.1) by an inverse Laplace transform,

$$
p_{24}(N)=\frac{1}{2 \pi i} \int_{\epsilon-i \pi}^{\epsilon+i \pi} d \beta e^{\beta(N-1)} \frac{16}{\Delta\left(e^{-\beta}\right)} .
$$


where the contour $C$ runs from $\epsilon-i \pi$ to $\epsilon+i \pi$, parallel to the imaginary axis. One may now take the high temperature limit $\epsilon \rightarrow 0$, and use the modular property of the discriminant function

$$
\Delta\left(e^{-\beta}\right)=\left(\frac{\beta}{2 \pi}\right)^{-12} \Delta\left(e^{-4 \pi^{2} / \beta}\right) .
$$

As $e^{-4 \pi^{2} / \beta} \rightarrow 0$, we can approximate $\Delta(q) \sim q$ and write the integral as

$$
p_{24}(N)=\frac{16}{2 \pi i} \int_{C} d \beta\left(\frac{\beta}{2 \pi}\right)^{12} e^{\beta(N-1)+4 \frac{\pi^{2}}{\beta}}
$$

This integral may be evaluated by steepest descent: the saddle point occurs at $\beta=$ $2 \pi / \sqrt{N-1}$, leading to the characteristic Hagedorn growth

$$
p_{24}(N) \sim \exp (4 \pi \sqrt{n w})
$$

for the spectrum of DH states.

To calculate the sub-leading terms systematically in an asymptotic expansion at large $N$, one may recognize that (6.5) is proportional to the integral representation of a modified Bessel function,

$$
\hat{I}_{\nu}(z)=-i(2 \pi)^{\nu} \int_{\epsilon-i \infty}^{\epsilon+i \infty} \frac{d t}{t^{\nu+1}} e^{\left(t+z^{2} / 4 t\right)} \hat{I}_{\nu}(z)
$$

We thus obtain

$$
\Omega(n, w)=p_{24}(N) \sim 2^{4} \hat{I}_{13}(4 \pi \sqrt{n w}) .
$$

Using the standard asymptotic expansion of $\hat{I}_{\nu}(z)$ at large $z$

$\hat{I}_{\nu}(z) \sim 2^{\nu}\left(\frac{z}{2 \pi}\right)^{-\nu-\frac{1}{2}}\left[1-\frac{(\mu-1)}{8 z}+\frac{(\mu-1)\left(\mu-3^{2}\right)}{2 !(8 z)^{2}}-\frac{(\mu-1)\left(\mu-3^{2}\right)\left(\mu-5^{2}\right)}{3 !(8 z)^{3}}+\ldots\right]$,

where $\mu=4 \nu^{2}$, we can compute the subleading corrections to the microscopic entropy of DH states to arbitrary high order,

$$
\log \Omega(n, w) \sim 4 \pi \sqrt{|n w|}-\frac{27}{4} \log |n w|+\frac{15}{2} \log 2-\frac{675}{32 \pi \sqrt{|n w|}}-\frac{675}{2^{8} \pi^{2}|n w|}-\ldots
$$

This is still not the complete asymptotic expansion of $\Omega(n, w)$ at large charge. Exponentially suppressed corrections to (6.8) can be computed by using the Rademacher formula (see [102] for a physicist account)

$$
\begin{aligned}
F_{\nu}(n)= & \sum_{c=1}^{\infty} \sum_{\mu=1}^{r} c^{w-2} \operatorname{Kl}(n, \nu, m, \mu ; c) \sum_{m+\Delta_{\mu}<0} F_{\mu}(m) \\
& \left|m+\Delta_{\mu}\right|^{1-w} \hat{I}_{1-w}\left[\frac{4 \pi}{c} \sqrt{\left|m+\Delta_{\mu}\right|\left(n+\Delta_{\nu}\right)}\right] .
\end{aligned}
$$


In this somewhat formidable expression, $F_{\mu}(m)$ denote the Fourier coefficients of a vector of modular forms

$$
f_{\mu}(\tau)=q^{\Delta_{\mu}} \sum_{m \geq 0} F_{\mu}(m) q^{m} \quad \mu=1, \ldots, r
$$

which transforms as a finite-dimensional unitary representation of the modular group of weight $w<0$, with

$$
\begin{aligned}
& f_{\mu}(\tau+1)=e^{2 \pi i \Delta_{\mu}} f_{\mu}(\tau) \\
& f_{\mu}(-1 / \tau)=(-i \tau)^{w} S_{\mu \nu} f_{\nu}(\tau)
\end{aligned}
$$

The coefficients $\operatorname{Kl}(n, \nu, m, \mu ; c)$ are generalized Kloosterman sums, defined as

$$
\mathrm{Kl}(n, \nu ; m, \mu ; c):=\sum_{0<d<c ; d \wedge c=1} e^{2 \pi i \frac{d}{c}\left(n+\Delta_{\nu}\right)} M\left(\gamma_{c, d}\right)_{\nu \mu}^{-1} e^{2 \pi i \frac{a}{c}\left(m+\Delta_{\mu}\right)}
$$

where

$$
\gamma_{c, d}=\left(\begin{array}{cc}
a & (a d-1) / c \\
c & d
\end{array}\right)
$$

is an element of $S l(2, \mathbb{Z})$ and $M(\gamma)$ its matrix representation. For $c=1$ in particular, we have:

$$
\mathrm{Kl}(n, \nu, m, \mu ; c=1)=S_{\nu \mu}^{-1}
$$

Going back to (6.11), we see that the growth of the Fourier coefficients is determined only by the Fourier coefficients of the "polar" part $F_{\mu}(m)$ where $m+\Delta_{\mu}<0$, as well as some modular data. The Ramanujan-Hardy formula

$$
F_{\mu}(n) \sim \exp \left[2 \pi \sqrt{\frac{c_{\text {eff }}}{6} n}\right]
$$

is reproduced by keeping the leading term $c=1$ only, using $\Delta=c_{\text {eff }} / 24, w=-c_{\text {eff }} / 2$ and the asymptotic behavior (6.9). The terms with $c>1$ also grow exponentially, but at a slower rate than the term with $c=1$. They therefore contribute exponentially suppressed contributions to $\log F_{\nu}(n)$.

Applying (6.11) to the case at hand, we have the convergent series expansion

$$
\Omega(n, w)=2^{4} \sum_{c=1}^{\infty} c^{-14} \operatorname{Kl}(n w+1,0 ; c) \hat{I}_{13}\left(\frac{4}{c} \pi \sqrt{|n w|}\right)
$$




\subsection{Macroscopic entropy and the topological amplitude}

We now turn to the macroscopic side, and determine the Bekenstein-Hawking-Wald entropy for a BPS black hole with the above charges. Since the attractor formalism is tailored for $\mathcal{N}=2$ supergravity, one should first decompose the spectrum in $\mathcal{N}=2$ multiplets: the $\mathcal{N}=4$ spectrum decomposes into one $\mathcal{N}=2$ gravity multiplet, 2 gravitino multiplets and $n_{V}=23$ vector multiplets (not counting the graviphoton). Provided the charges under the 4 vectors in the gravitino multiplets vanish, the $\mathcal{N}=2$ attractor mechanism applies.

The topological amplitude $F_{1}$ has been computed in [103], and can be obtained as the holomorphic part of the $R^{2}$ amplitude at one-loop,

$$
f_{R^{2}}=24 \log \left(T_{2}|\eta(T)|^{4}\right)
$$

where $T, U$ denote the Kähler and complex structure moduli of the torus $T^{2}$. All higher topological amplitudes $F_{g}$ for $g>1$ vanish for models with $\mathcal{N}=4$ supersymmetry. We therefore obtain the generalized prepotential

$$
F\left(X^{I}, W^{2}\right)=-\frac{1}{2} \sum_{a, b=2}^{23} C_{a b} \frac{X^{a} X^{b} X^{1}}{X^{0}}-\frac{W^{2}}{128 \pi i} \log \Delta(q)
$$

where $C_{a b}$ is the intersection matrix on $H^{2}(K 3), T=X^{1} / X^{0}$ and $q=e^{2 \pi i T}$. The appearance of the same discriminant function $\Delta(q)$ as in the microscopic heterotic counting (6.1) is at this stage coincidental.

Identifying $p^{1}=w, q_{0}=n$ and allowing for arbitrary electric charges $q_{0}, q_{i=2 . .23}$, the black hole free energy (5.17) reduces to

$$
\mathcal{F}\left(\phi^{I}, p^{I}\right)=-\frac{\pi}{2} C_{a b} \frac{\phi^{a} \phi^{b} p^{1}}{\phi^{0}}-\log |\Delta(q)|^{2}
$$

where

$$
q=\exp \left[\frac{2 \pi}{\phi^{0}}\left(p^{1}+i \phi^{1}\right)\right]
$$

The Bekenstein-Hawking-Wald entropy is then obtained by performing a Legendre transform over all electric potentials $\phi^{I}, I=0, \ldots 23$. The Legendre transform over $\phi^{a=2 . .23}$ sets $\phi^{a}=\left(\phi^{0} / p^{1}\right) C^{a b} q_{b}$, where $C^{a b}$ is the inverse of the matrix $C_{a b}$. We will check a posteriori that in the large charge limit, it is consistent to approximate $\Delta(q) \sim q$, whereby all dependence on $\phi^{1}$ disappears. We thus obtain

$$
S_{B H W} \sim\left\langle\left[-\frac{\pi}{2} \frac{C^{a b} q_{a} q_{b}}{p^{1}} \phi^{0}+4 \pi \frac{p^{1}}{\phi^{0}}+\pi \phi^{0} q_{0}\right]\right\rangle_{\phi^{0}}
$$


The extremum of the bracket lies at

$$
\phi_{*}^{0}=\frac{1}{2} \sqrt{-p^{1} / \hat{q}_{0}}, \quad \hat{q}_{0}:=q_{0}+\frac{1}{2 p^{1}} C^{a b} q_{a} q_{b}
$$

so that at the horizon the Kähler class $\operatorname{Im} T \sim \sqrt{-p^{1} \hat{q}_{0}}$ is very large, justifying our assumption. Evaluating (6.24) at the extremum, we find

$$
S_{B H} \sim 4 \pi \sqrt{Q^{2} / 2}, \quad Q^{2}=2 p^{1} q_{0}+C^{a b} q_{a} q_{b}
$$

in agreement with the leading exponential behavior in (6.10), including the precise numerical factor. Note that the argument up to this stage is independent of the OSV conjecture, and relies only on the classical attractor mechanism in the presence of higher-derivative corrections. This observation, first made in [99], indicates that the tree-level $R^{2}$ coupling in the effective action of the heterotic string on $T^{6}$ (or, equivalently, large volume limit of the 1 -loop $R^{2}$ coupling in type IIA $/ K 3 \times T^{2}$ ) is sufficient to cloak the singularity of the small black hole behind a smooth horizon. This is in fact confirmed by a study of the corrected geometry [104, 105].

\subsection{Testing the OSV Formula}

We are now ready to test the proposal (5.20) and evaluate the inverse Laplace transform of $\exp (\mathcal{F})$ with respect to the electric potentials,

$$
\Omega_{O S V}(p)=\int d \phi^{0} d \phi^{1} d^{22} \phi^{a} \frac{1}{|\Delta(q)|^{2}} \exp \left[-\frac{\pi}{2} C_{a b} \frac{\phi^{a} \phi^{b} p^{1}}{\phi^{0}}+\pi \phi^{0} q_{0}+\pi \phi^{a} q_{a}\right]
$$

Due to the non-definite signature of $C_{a b}$, the integral over $\phi^{a}$ diverges for real values. This may be avoided by rotating the integration contour to $\epsilon+i \mathbb{R}$ for all $\phi$ 's. The integral over $\phi^{a}$ is now a Gaussian, leading to

$$
\Omega_{O S V}(Q)=\int d \phi^{0} d \phi^{1}\left(\frac{\phi_{0}}{p^{1}}\right)^{11} \frac{1}{|\Delta(q)|^{2}} \exp \left(-\frac{1}{2} \frac{C^{a b} q_{a} q_{b}}{p^{1}} \phi^{0}+q_{0} \phi^{0}\right)
$$

where we dropped numerical factors and used the fact that $\operatorname{det} C=1$. The asymptotics of $\Omega$ is independent of the details of the contour, as long as it selects the correct classical saddle point (6.25) at large charge. Approximating again $\Delta(q) \sim q$, we find the quantum version of $(6.24)$,

$$
\Omega_{O S V}(Q) \sim \int d \phi^{0} d \phi^{1}\left(\frac{\phi_{0}}{p^{1}}\right)^{11} \exp \left(-\frac{1}{2} \frac{C^{a b} q_{a} q_{b}}{p^{1}} \phi^{0}-4 \pi \frac{p^{1}}{\phi^{0}}+q_{0} \phi^{0}\right)
$$

The integral over $\phi^{1}$ superficially leads to an infinite result. However, since the free energy is invariant under $\phi^{1} \rightarrow \phi^{1}+\phi^{0}$, it is natural to restrict the integration to a 
single period $\left[0, \phi^{0}\right]$, leading to an extra factor of $\phi^{0}$ in (6.29). The integral over $\phi^{0}$ is now of Bessel type, leading to

$$
\Omega_{O S V}(Q)=\left(p^{1}\right)^{2} \hat{I}_{13}\left(4 \pi \sqrt{Q^{2} / 2}\right)
$$

in impressive agreement with the microscopic result (6.8) at all orders in $1 / Q$.

Some remarks on this computation are in order:

- Note that the extra factor $\left(p^{1}\right)^{2}$ in Equation (6.30) is inconsistent with $S O(6,22, \mathbb{Z})$ duality, which requires the exact degeneracies to be a function of $Q^{2}$ only. Moreover, the agreement depends crucially on discarding the non-holomorphic correction proportional to $\log T_{2}$ in $F_{1}$. Both of these issues call for a better understanding of the relation between the physical amplitude and the topological wave function in the real polarization. It should be mentioned that an alternative approach has been developped by Sen, keeping the non-holomorphic corrections but using a different statistical ensemble [106, 107].

- The "all order" result (6.30) depends only on the number of $\mathcal{N}=2$ vector multiplets, as well as on the leading large volume behavior of $F_{1} \sim q /(128 \pi i)$. By heterotic/type II duality, this term is mapped to a tree-level $R^{2}$ interaction on the heterotic side, which is in fact universal. We thus conclude that in all $\mathcal{N}=2$ models which admit a dual heterotic description, provided higher genus $F_{g>1}$ and genus 0,1 Gromov-Witten instantons can be neglected, the degeneracies of small black holes predicted by (5.20) are given by

$$
\Omega_{O S V}(Q) \propto \hat{I}_{\frac{n_{V}+3}{2}}\left(4 \pi \sqrt{Q^{2} / 2}\right)
$$

where $n_{V}$ is the number of Abelian gauge fields, including the graviphoton. We return to the validity of the assumption in the next subsection.

Exercice 16 By applying a similar argument to large black holes with $p^{0}=0$, assuming that only the large-volume limit of $F_{1}$ contributes, show that the OSV conjecture (5.20), in the saddle point approximation, predicts [70, 64]

$$
\Omega\left(p^{A}, q_{A}\right) \sim \pm \frac{1}{2}\left|\operatorname{det} C_{a b}(p)\right|^{-1 / 2}(\hat{C}(p) / 6)^{\frac{n_{V}+2}{2}} \times \hat{I}_{\frac{n_{V}+2}{2}}\left(2 \pi \sqrt{-\hat{C}(p) \hat{q}_{0} / 6}\right)
$$

where

$$
C_{A B}(p)=C_{A B C} p^{C}, \quad C(p)=C_{A B C} p^{A} p^{B} p^{C}, \quad \hat{C}(p)=C(p)+c_{2 A} p^{A},
$$

and compare to the microscopic counting (2.19). 
- In order to see if the strong version of the OSV conjecture has a chance to hold, it is instructive to change variable to $\beta=\pi / t$ in (6.3) and rewrite the exact microscopic result as

$$
\Omega_{\text {exact }}(Q)=\int d t t^{-14} \frac{\exp \left(\frac{\pi n w}{t}\right)}{\Delta\left(e^{-4 \pi t}\right)}
$$

On the other hand, it is convenient to change variables in the OSV integral (6.28) to $\tau_{1}=\phi^{1} / \phi^{0}, \tau_{2}=-p^{1} / \phi^{0}$, with Jacobian $d \phi^{0} d \phi^{1}=8\left(p^{1}\right)^{2} d \tau_{1} d \tau_{2} / \tau_{2}^{3}$, leading to

$$
\Omega_{O S V}(Q) \sim \int d \tau_{1} d \tau_{2} \tau_{2}^{-14} \frac{\exp \left(\frac{\pi n w}{\tau_{2}}\right)}{\left|\Delta\left(e^{-2 \pi \tau_{2}+2 \pi i \tau_{1}}\right)\right|^{2}}
$$

Despite obvious similarities, it appears unlikely that the two results are equal non-perturbatively.

- Just as the perturbative result (6.8), the result (6.30) misses subleading terms in the Rademacher expansion (6.19). It does not seem possible to interpret any of the terms with $c>1$ as the contribution of a subleading saddle point in either (6.5) or (6.28).

Despite these difficulties, it is remarkable that the black hole partition function in the OSV ensemble, obtained from purely macroscopic considerations, reproduces the entire asymptotic series exactly to all orders in inverse charge.

\section{$6.4 \mathcal{N}=2$ Orbifolds}

We conclude this section with a few words on small black holes in $\mathcal{N}=2$ orbifolds, referring to $[70,64]$ for detailed computations. We find that the agreement found in $\mathcal{N}=4$ models broadly continues to hold, with the following caveats:

- In contrast to $\mathcal{N}=4$ cases, the neglect of Gromov-Witten instantons is harder to justify rigorously: when $\chi(X) \neq 0$, the series of point-like instantons contribution becomes strongly coupled in the regime of validity of the Rademacher formula, $\hat{q}_{0} \gg \hat{C}(p)$. The strong coupling behavior is controlled, up to a logarithmic term, by the Mac-Mahon function (4.33), which is exponentially suppressed in this regime. The logarithmic term in (4.37) may be reabsorbed into a redefinition of the topological string amplitude $\Psi_{\text {top }} \rightarrow \lambda^{\chi / 24} \Psi_{\text {top }}$. As for non-degenerate instantons, they are exponentially suppressed provided all magnetic charges are non zero. This is unfortunately not the case for the small black holes dual to the heterotic DH states, whose Kähler classes are attracted to the boundary of the Kähler cone at the horizon. 
- For BPS states in twisted sectors of $\mathcal{N}=2$ orbifolds, we find that the instantondeprived OSV proposal appears to successfully reproduce the absolute degeneracies, equal to the indexed degeneracies, to all orders. For untwisted DH states of the OSV prediction appears to agree with the absolute degeneracies of untwisted DH states to leading order ( which have the same exponential growth as twisted DH states), but not at subleading order (as the subleading corrections in the untwisted sector are moduli-dependent, and uniformly smaller than in the twisted sectors). The indexed degeneracies are exponentially smaller than absolute degeneracies, due to cancellations of pairs of DH states, so plainly disagree with the OSV prediction.

\section{Quantum Attractors and Automorphic Partition Functions}

In this final chapter, we investigate an interesting proposal by Ooguri, Verlinde and Vafa [41], to interpret the OSV conjecture as a holographic duality between the Hilbert space of black hole micro-states in usual time-like quantization, and the Hilbert space of spherically symmetric geometries in a radial quantization scheme. As we shall explain, this approach naturally suggests a relation between exact black hole degeneracies and Fourier coefficients of certain automorphic forms, reminiscent of the DVV proposal discussed in Section 2.23, which may be viewed as a non-perturbative generalization of the OSV conjecture [108].

\subsection{OSV Conjecture and Quantum Attractors}

In order to motivate this approach, recall that, after analytically continuing $\phi^{I}=i \chi^{I}$ to the imaginary axis, the r.h.s.of the OSV conjecture (5.20)

$$
\Omega\left(p^{I}, q_{I}\right) \sim \int d \chi^{I} \Psi_{\mathrm{top}}^{*}\left(p^{I}+\chi^{I}\right) \Psi_{\mathrm{top}}\left(p^{I}-\chi^{I}\right) e^{i \pi \chi^{I} q_{I}}:=W_{\Psi_{\mathrm{top}}}\left(p^{I}, q_{I}\right)
$$

could be interpreted as the Wigner distribution associated to the wave function $\Psi_{\text {top }}$. In usual quantum mechanics, the Wigner distribution $W_{\psi}(p, q)$ is a function on phase space associated to a wave function $\psi(q)$, such that quantum averages of Weyl-ordered operators on $\psi$ are equal to classical averages of their symbols with respect to $W_{\psi}$,

$$
\langle\psi|\mathcal{O}(\hat{p}, \hat{q})| \psi\rangle=\int d p d q W_{\psi}(p, q) \mathcal{O}(p, q)
$$

Moreover, when $\psi$ satisfies the Schrödinger equation, $W$ satisfies the classical Liouville equation to leading order in $\hbar$; the Wigner distribution is thus a useful tool to study the semi-classical limit. The above observation thus begs the question: what is the 
physical quantum system of which $\Psi_{\text {top }}$ is the wave function ${ }^{16}$, and how does it encode the black hole degeneracies?

Exercice 17 Show that

$$
W_{\tilde{\psi}}\left(p^{I}, q_{I}\right)=W_{\psi}\left(q_{I}, p^{I}\right)
$$

where $\tilde{\psi}$ is the Fourier transform of $\psi$.

In order to try and answer this question, it is useful to reabsorbe the dependence on the charges $\left(p^{I}, q_{I}\right)$ into the state itself, by defining

$$
\Psi_{p, q}(\chi):=e^{i q \chi} \Psi_{\text {top }}(\chi-p):=V_{p, q} \cdot \Psi_{\text {top }}(\chi)
$$

Equation (7.2) is then rewritten more suggestively as an overlap of two wave functions,

$$
\Omega(p, q) \sim \int d \chi \Psi_{p, q}^{*}(\chi) \Psi_{p, q}(\chi)
$$

On the other hand, recall that the near horizon geometry $A d S_{2} \times S_{2}$, written in global coordinates as

$$
d s^{2}=|Z|^{2}\left(\frac{-d \tau^{2}+d \sigma^{2}}{\cos ^{2} \sigma}+d^{2} \Omega\right)
$$

has two different boundaries at $\sigma=0, \pi$, respectively; its Euclidean sections at finite temperature have the topology of a cylinder (see Figure 2).

Exercice 18 The metric (7.6) is equivalent to (2.13) upon changing coordinates $\tau=$ $\arctan (z+t)-\arctan (z-t), \quad \sigma=\arctan (z+t)+\arctan (z-t)$. Map out the portion of the global geometry covered by the Poincaré coordinates $z, t$.

With this in mind, it is tempting to view (7.5) as an analogue of open/closed duality for conformal field theory on the cylinder,

$$
\operatorname{Tr} e^{-\pi t H_{\text {open }}}=\left\langle B^{\prime}\left|e^{-\frac{\pi}{t} H_{\text {closed }}}\right| B\right\rangle
$$

where $|B\rangle$ and $\left|B^{\prime}\right\rangle$ are closed string boundary states. The r.h.s. of (7.5), analogue of the closed string channel, is identified as the partition function of quantum gravity on $A d S^{2} \times S^{2}$ in radial quantization from $\sigma=0$ to $\sigma=\pi$, with boundary conditions specified by the "boundary states" $\Psi_{p, q} \Psi_{p, q}^{*}$; the l.h.s. is identified as a trace in the Hilbert space of "open string" degrees of freedom, corresponding to the usual time-like quantization along $\tau$. The fact that there is no analogue of the Hamiltonians $H_{\text {open }}$

\footnotetext{
${ }^{16}$ Or, to paraphrase Ford Prefect, what is the Question to the Answer $\Psi_{\text {top }}$ ?
} 
and $H_{\text {closed }}$ in (7.2) should cause no worry since, in diffeomorphism invariant theories, physical states have to be solutions of the Wheeler-De Witt equation $H|\psi\rangle=0$.

In order for this interpretation to make sense, it should be possible to view $\Psi_{\text {top }}$ as a state in the Hilbert space for radial quantization. Since, as explained in Section (4.4), $\Psi_{\text {top }}$ lives in a Hilbert space of finite functional dimension, it certainly cannot describe the full quantum state in radial quantization, but at best a "mini-superspace" truncation ${ }^{17}$ thereof, where only static, spherically symmetric BPS geometries are kept. We are thus lead to the problem of quantizing the attractor flow (3.27),(3.28), and showing that the resulting "BPS" Hilbert space is the correct habitat for $\Psi_{\text {top }}$. On top of this, an outstanding question is to find a physical principle that selects $\Psi_{\text {top }}$ among the infinite number of states in the BPS Hilbert space.

Several remarks about this approach are in order:

- This heuristic reasoning agrees with the usual AdS/CFT intuition that the black hole micro-states should be described by "gauge theoretical" degrees of freedom living on the boundary of $A d S_{2}$, which would holographically describe the gravitational physics in the bulk. Contrary to higher dimensional AdS spaces, the conformal quantum mechanics describing $A d S_{2}$ is still largely mysterious, but the above reasoning suggests that one should be able to have indirect access to the CFT spectrum, by relating it to an overlap of wave functions in the Hilbert space of radially-quantized AdS. At the risk of belaboring, let us stress that the two Hilbert spaces are different, just like the open string and closed string Hilbert spaces are different.

- The idea of radial quantization of static black holes already has a long history in the canonical gravity literature, e.g. [109, 110, 111, 112, 113, 114]. One novelty here is the embedding in a supersymmetric context, where the mini-superspace approximation might be under better control. Furthermore, holography is a new ingredient which supports the idea that the spectrum of the global time Hamiltonian can be reconstructed from the radial wave functions. Let us also note that the idea of quantizing the BPS sector configurations has been applied successfully in various set-ups $[115,116,117,118]$, and in particular to the problem of two-charge black holes [119].

- The proposal turns the question whether $\Omega(p, q)$ corresponds to absolute or index degeneracies into a choice of boundary conditions for fermions along the thermal

\footnotetext{
${ }^{17}$ One may hope that such a truncation may be innocuous for computing indexed degeneracies of BPS black holes, but this needs to be justified a posteriori.
} 
circle. It is unlikely that the mini-superspace truncation can hold true with supersymmetry-breaking boundary conditions, which favors the second option.

- One usually assumes that black hole micro-states can be described only in terms of the near horizon geometry. The above proposal to quantize the whole attractor flow seems to be at odds with this idea. A possible way out is that the topological wave function is a fixed point of the quantum attractor flow. In the sequel, we will study the full quantum attractor flow, from asymptotic infinity to the horizon, as a function of the Poincaré radial coordinate $r$ (rather than the "global radial coordinate $\sigma "$, which is well defined only near the horizon).

- The analogy between global $A d S_{2}$ and open strings goes in fact further: due to pair production of charged particles, a black hole may fragment in different throats, analogous to the joining and splitting interactions of open strings [120] (see [121] for a perturbative approach to this problem). It has been suggested (and observed in certain non-compact Calabi-Yau threefolds) that the attractor flow should be "second quantized" to allow for this possibility [95]. It is unclear whether there exists a black hole analogue of the the process whereby the two ends of an open string join to form a closed string.

- Finally, let us mention some further interest for this issue arising from the analogy between black hole attractor equations and the equations that determine supersymmetric vacua in flux compactifications (see e.g. [12] for a recent discussion). Upon double analytic continuation, one may hope to relate the black hole wave function to a wave function of the Universe, and address vacuum selection in the Landscape [41]. There are however many difficulties with this idea that we shall not discuss here.

We now turn to a detailed discussion of the quantization of the attractor flow, which we achieve by showing its equivalence with the quantization of BPS geodesic motion.

\subsection{Attractor Flows and Geodesic Motion}

The most convenient way to perform radial quantization is to reformulate the attractor flow equations discussed in Section 3 as geodesic motion on a larger space [108]. For this purpose, let us generalize the ansatz (3.25) to allow for general stationary metrics and gauge connections,

$$
d s_{4}^{2}=-e^{2 U}(d t+\omega)^{2}+e^{-2 U} \gamma_{i j} d x^{i} d x^{j}, \quad A_{4}^{I}=\zeta^{I} d t+A_{i}^{I} d x^{i}
$$

where $\gamma_{i j}, U, \omega, A_{i}^{I}, \zeta^{I}$, together with the four-dimensional scalars $z^{i} \in \mathcal{M}_{4}$, are independent of time. The four-dimensional equations of motion can then be expressed in terms 

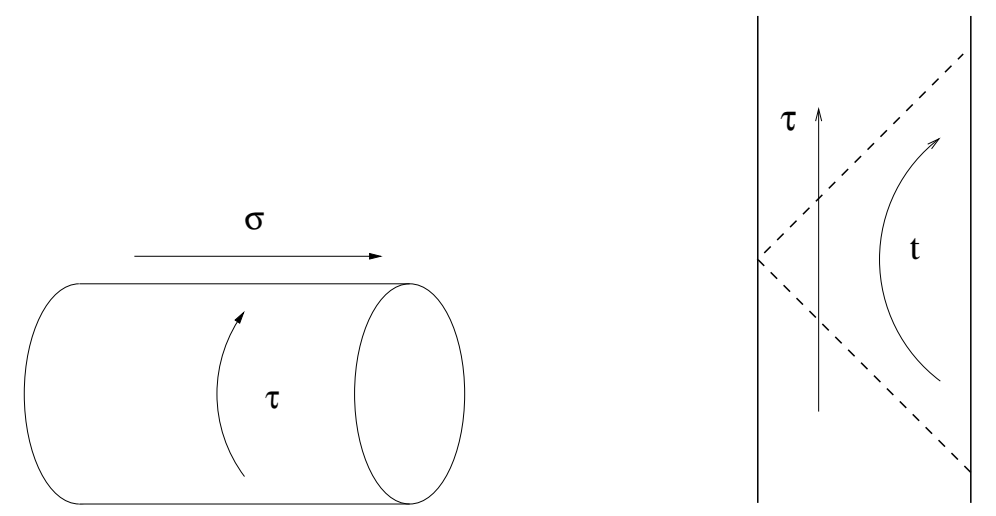

Figure 2: Left: the cylinder amplitude in string theory can be viewed either as a trace over the open string Hilbert space (quantizing along $\tau$ ) channel) or as an inner product between two wave functions in the closed string Hilbert space (quantizing along $\sigma$ ). Right: The global geometry of Lorentzian $A d S_{2}$ has the topology of a strip; its Euclidean continuation at finite temperature becomes a cylinder. $\tau$ and $t$ are the global and Poincaré time, respectively.

a field theory in 3 Euclidean dimensions. Since the isometric direction $t$ is time-like, we refer to this reduction as $K K^{*}$ reduction.

In the usual Kaluza-Klein reduction, the one-forms $A_{i}^{I}$ and $\omega$ can be dualized into axionic scalars $\tilde{\zeta}_{I}, a$, using the usual duality " $\star d \omega=d a$ " between one-forms and pseudoscalars in three dimensions; together with $z^{i}, \zeta^{I}$, these scalars make a larger Riemannian space $\mathcal{M}_{3}$. The 4D Einstein-Maxwell equations then reduce to gravity in $2+1$ dimensions, coupled to a non-linear sigma model on $\mathcal{M}_{3}$. The $\mathrm{KK}^{*}$ reduction is similar,

$$
S=\int d^{3} x \sqrt{\gamma}\left(R[\gamma]-g_{a b} \gamma^{i j} \partial_{i} \phi^{a} \partial_{j} \phi^{b}\right)
$$

except that the scalar fields $\phi^{a}$ live in a pseudo-Riemannian space $\mathcal{M}_{3}^{*}$ with metric $g_{a b}$, with non-positive definite signature $[122,123] . \mathcal{M}_{3}^{*}$ is simply related $\mathcal{M}_{3}$ by analytic continuation $\left(\zeta^{I}, \tilde{\zeta}_{I}\right) \rightarrow i\left(\zeta^{I}, \tilde{\zeta}_{I}\right)$. We shall give explicit examples of $\mathcal{M}_{3}^{*}$ for $\mathcal{N}=2$ supergravities in the next subsections.

One important feature of $\mathcal{M}_{3}^{*}$ is that it always possesses $2 n+2$ isometries corresponding to the gauge symmetries of $A^{I}, \tilde{A}_{I}, \omega$, as well as rescalings of time $t$. The Killing vector fields satisfy the Lie-bracket algebra

$$
\left[p^{I}, q_{J}\right]=2 \delta_{J}^{I} k, \quad\left[m, p^{I}\right]=p^{I},\left[m, q_{I}\right]=q_{I},[m, k]=2 k
$$

The notation anticipates the fact that the conserved charges associated to these isometries will be identified with the electric and magnetic charges, NUT charge and ADM mass of the black hole. 
Now, let us restrict to spherically symmetric, stationary solutions: the spatial slices can be parameterized as

$$
\gamma_{i j} d x^{i} d x^{j}=N^{2}(\rho) d \rho^{2}+r^{2}(\rho) d \Omega_{2}^{2}
$$

while all scalars on $\mathcal{M}_{3}^{*}$ become functions of $\rho$ only. The three-dimensional sigma-model action reduces to the classical mechanics

$$
S=\int d \rho\left[\frac{N}{2}+\frac{1}{2 N}\left(r^{\prime 2}-r^{2} g_{a b} \phi^{\prime a} \phi^{\prime b}\right)\right]
$$

where the prime denotes a derivative with respect to $\rho$, and we dropped a total derivative term. This Lagrangian describes the free motion of a fiducial particle on a cone ${ }^{18}$ $\mathcal{C}=\mathbb{R}^{+} \times \mathcal{M}_{3}^{*}$ over the three-dimensional moduli space $\mathcal{M}_{3}$. The lapse $N$ is an auxiliary field; its equation of motion enforces the mass shell condition

$$
r^{\prime 2}-r^{2} g_{a b} \phi^{\prime a} \phi^{\prime b}=N^{2}
$$

or equivalently, the Hamiltonian constraint

$$
H_{W D W}=\left(p_{r}\right)^{2}-\frac{1}{r^{2}} g^{a b} p_{a} p_{b}-1 \equiv 0
$$

Provided one enforces this condition, one may impose the gauge choice $N=1$ in (7.12). Solutions are thus massive geodesics on the cone, with fixed mass equal to 1. The motion separates into geodesic motion on $\mathcal{M}_{3}^{*}$, with affine parameter $\tau$ such that $d \tau=d \rho / r(\rho)^{2}$, and motion along the radial direction $r$, governed by the conformal quantum mechanics Hamiltonian [126],

$$
\left(p_{r}\right)^{2}-\frac{C^{2}}{r^{2}}-1 \equiv 0, \quad g^{i j} p_{i} p_{j} \equiv C^{2}
$$

where $p_{r}=r^{\prime}=\dot{r} / r^{2}$ and $p_{i}=r^{2} \phi^{\prime i}=\dot{\phi}^{i}$; here the dot denotes a derivative with respect to $\tau$.

\subsubsection{Conserved charges}

As anticipated in (7.10), the isometries of $\mathcal{M}_{3}$ imply conserved Noether charges, identified as the electric, magnetic and NUT charges $p^{I}, q_{I}, k$. Their Poisson bracket reflect the Lie algebra of the isometries,

$$
\left\{p^{I}, q_{J}\right\}=2 \delta_{J}^{I} k, \quad \text { etc. }
$$

\footnotetext{
${ }^{18} \mathrm{~A}$ similar system arises in mini-superspace cosmology [124, 125].
} 
In particular, the electric and magnetic charges satisfy an Heisenberg algebra, the center of which is the NUT charge $k$. The latter is related to the off-diagonal term in the metric (7.8) via $\omega=k \cos \theta d \phi$. When $k \neq 0$, the metric

$$
d s_{4}^{2}=-e^{2 U}(d t+k \cos \theta d \phi)^{2}+e^{-2 U}\left[d \rho^{2}+r^{2}\left(d \theta^{2}+\sin ^{2} \theta d \phi^{2}\right)\right]
$$

has closed timelike curves (CTC) near $\theta=0$, all the way from infinity to the horizon. Bona fide 4D black holes therefore need to have $k=0$, a kind of classical limit for the Heisenberg algebra (7.16). In the following, we shall nevertheless retain $k \neq 0$, as it will turn out to greatly extend the symmetries of the problem. Eventually, one should always return to the case $k=0$.

\subsubsection{Extremal black holes and light-like geodesics}

In order to understand the meaning of the integration constant $C$ in (7.15), note that the first equation may be integrated in terms of the affine parameter $\tau$ to yield

$$
r=\frac{C}{\sinh (C \tau)}, \quad \rho=\frac{C}{\tanh C \tau}
$$

Assuming that the sphere $S^{2}$ reaches a finite area $A$ at the horizon $\tau=\infty$, so that $e^{-2 U} r^{2} \rightarrow A /(4 \pi)$, the metric (7.8) may be rewritten as [127]

$$
d s^{2} \sim \frac{C^{2}}{\sinh ^{2}(C \tau)}\left(-\frac{4 \pi}{A}(d t+\omega)^{2}+\frac{A}{4 \pi} d \tau^{2}\right)+\frac{A}{4 \pi} d^{2} \Omega
$$

The horizon at $\tau=\infty$ is degenerate for $C^{2}=0$, and non-degenerate for $C^{2}>0$, corresponding to extremal and non-extremal black holes, respectively. We conclude that extremal black holes correspond to light-like geodesics on $\mathcal{M}_{3}^{*}$. It is indeed fortunate that $\mathcal{M}_{3}^{*}$ is a pseudo-Riemannian manifold, so that light-like geodesics do exist.

Exercice 19 Show that the extremality parameter $C$ is related to the BekensteinHawking entropy and Hawking temperature by $C^{2}=2 S_{B H} T_{H}$.

Setting $C=0$ in (7.15), we moreover see that $r=\rho=1 / \tau$, and therefore that the spatial slices in the ansatz (7.8) are flat. We could therefore have set $N=1, r=1 / \tau$ from the start, and obtained the action for geodesic motion on $\mathcal{M}_{3}$ in affine parameterization,

$$
S=\int d \tau \frac{1}{2} g_{a b} \dot{\phi}^{a} \dot{\phi}^{b}
$$

Retaining the variable $r$ may however be important for defining observables such as the horizon area, $A_{H}=\left.e^{-2 U} r^{2}\right|_{U \rightarrow-\infty}$ and the ADM mass $M_{A D M}=\left.r\left(e^{2 U}-1\right)\right|_{U \rightarrow 0}$. 


\subsubsection{BPS Black Holes and BPS Geodesics}

In order to determine which extremal back holes are supersymmetric, it is necessary to reduce the full $D=4$ SUGRA on the stationary, spherically symmetric ansatz. One thus obtains the Lagrangian for a spinning particle moving on $\mathcal{M}_{3}^{*}$, schematically ${ }^{19}$

$$
S=\int d \tau\left[g_{a b} \dot{\phi}^{a} \dot{\phi}^{b}+g_{a b} \psi^{a} D_{\tau} \psi^{b}+R_{a b c d} \psi^{a} \psi^{b} \psi^{c} \psi^{d}\right]
$$

where $D_{\tau}$ contains the spin connection. This Lagrangian is invariant under supersymmetry transformations of the form

$$
\delta \phi^{a}=O(\psi), \quad \delta \psi^{A \alpha}=V_{a}^{A \beta} \dot{\phi}^{a} \epsilon_{\beta}^{\alpha}+O\left(\psi^{2}\right)
$$

where we used the special holonomy of $\mathcal{M}_{3}^{*}$ to convert the vector index $a$ on $\psi$ into a pair of indices $A \alpha$ (we shall be more precise about the nature of these indices in the next subsections). The object $V_{a}^{A \alpha}$ is a viel-bein on $\mathcal{M}_{3}^{*}$, which converts the one-form index $a$ into $A \alpha$. Thus one can think of $P^{A \alpha}=V_{a}^{A \alpha} \dot{\phi}^{a}$ as the momentum of the fiducial particle on $\mathcal{M}_{3}^{*}$. The black hole solution is BPS if there exists $\epsilon_{\beta}^{\alpha} \neq 0$ such that $\delta \psi^{A \alpha}=0$ : this is equivalent to the viel-bein $P$ having one or more zero eigenvectors. This implies in particular that the momentum $P$ is light-like, but is a more stringent condition. In theories with $N \geq 2$ supersymmetry in 4 dimensions, black holes preserving different fractions of supersymmetry will correspond to different orbits of the momentum $P$, distinguished according to their isotropy group.

\subsubsection{Radial flow in $\mathcal{N}=8$ and $\mathcal{N}=4$ SUGRA}

In order to make the discussion more concrete, let us consider the maximally supersymmetric case, namely black holes in $\mathcal{N}=8$ SUGRA. The moduli space in 4 dimensions is the 70-dimensional symmetric space $E_{7(7)} / S U(8)$, where $E_{7(7)}$ is the split real form ${ }^{20}$ of the exceptional group $E_{7}$ (see [128] for a review of U-duality). Upon KK reduction 3 dimensions, one obtains the 128-dimensional spaces

$$
\mathcal{M}_{4}=\frac{E_{7(7)}}{S U(8)} \rightarrow \quad \mathcal{M}_{3}=\frac{E_{8(8)}}{S O(16)}, \quad \mathcal{M}_{3}^{*}=\frac{E_{8(8)}}{S O^{*}(16)}
$$

where $S O^{*}(16)$ is the real form of $S O(16)$ with maximal non-compact group $U(8)$. The supersymmetry variation of the fermions is

$$
\delta \lambda_{A}=\epsilon_{I} \Gamma_{A \dot{A}}^{I} P^{\dot{A}}
$$

\footnotetext{
${ }^{19}$ For simplicity, we drop the cone direction $r$ in this discussion.

${ }^{20}$ The number in parenthesis indicates the signature of the real form, i.e. the difference of the number of non-compact and compact generators, $70-63=7$ in this case.
} 
where the SUSY parameter $\epsilon_{I}$ is in a vector of the R-symmetry group $S O^{*}(16)$, the momentum $P^{\dot{A}}$ is a 128 spinor of $S O^{*}(16)$ (corresponding to the tangent space to $\left.E_{8(8)} / S O^{*}(16)\right)$, and $\lambda_{A}$ is a conjugate spinor. The smallest orbit of the spinor of $S O^{*}(16)$, which defines Cartan's pure spinor ${ }^{21}$ has dimension 58 , and is associated to 1/2-BPS trajectories. 1/4-BPS and 1/8-BPS black holes are associated to 92 and 114 dimensional orbits of spinors of lesser purity. As we shall see in Section 7.4.3 below, these $S O(16)$ orbits are in one-to-one relation with the nilpotent co-adjoint orbits of $E_{8(8)}$. This is a manifestation of the general Kostant-Sekiguchi correspondence between nilpotent co-adjoint orbits of $G(\mathbb{R})$ and nilpotent orbits of $K(\mathbb{C})$ [129, 130].

Similarly, the moduli space of $\mathcal{N}=4$ SUGRA with $n_{v}$ vector multiplets was given in (2.21) above. After compactification to three dimensions, one obtains

$$
\mathcal{M}_{3}=\frac{S O\left(8, n_{v}+2\right)}{S O(8) \times S O\left(n_{v}+2\right)}, \quad \mathcal{M}_{3}^{*}=\frac{S O\left(8, n_{v}+2\right)}{S O(6,2) \times S O\left(2, n_{v}\right)}
$$

The fermionic SUSY variation is

$$
\delta \lambda_{A}^{a}=\epsilon_{I} \Gamma_{A \dot{A}}^{I} P^{\dot{A} a}
$$

where $\epsilon_{I}$ is a vector of the R-symmetry group $S O(6,2)$, and $P^{\dot{A} a}\left(a=1 \ldots n_{v}\right)$, are a collection of $n_{v}$ spinors of $S O(6,2)$ corresponding to the tangent space of $S O\left(8, n_{v}+\right.$ $2) / S O(6,2) \times S O\left(2, n_{v}\right)$. Supersymmetric solutions can be obtained by requiring that the momentum factorizes into $P^{\dot{A} a}=\lambda^{\dot{A}} v^{a}$. 1/2 SUSY trajectories correspond to pure spinors of $S O(6,2)$.

\subsubsection{Attractor Flow in $\mathcal{N}=2$ SUGRA and the $c^{*}$-map}

In this section, we apply this framework to the case of BPS black holes in $D=4 \mathcal{N}=2$ supergravity, and derive the attractor equations which were postulated in Section 3.3.

For this purpose, we reduce the $D=4$ SUGRA action to 3 dimensions along a time-like direction. In the usual space-like case, the answer is well known: the threedimensional space is a quaternionic-Kähler space, known as the "c-map" of the special Kähler manifold $\mathcal{M}_{V}$ describing the vector multiplet scalars in 4 dimensions [131, 132]. The effect of the $K K^{*}$ reduction is to analytically continue $\left(\zeta^{I}, \tilde{\zeta}_{I}\right) \rightarrow i\left(\zeta^{I}, \tilde{\zeta}_{I}\right)$, leading to the metric ${ }^{22}$

$$
\begin{aligned}
& d s^{2}=2(d U)^{2}+g_{i \bar{j}}(z, \bar{z}) d z^{i} d z^{\bar{j}}+\frac{1}{2} e^{-4 U}\left(d a+\zeta^{I} d \tilde{\zeta}_{I}-\tilde{\zeta}_{I} d \zeta^{I}\right)^{2} \\
& +e^{-2 U}\left[(\operatorname{Im} \mathcal{N})_{I J} d \zeta^{I} d \zeta^{J}+\left(\operatorname{Im} \mathcal{N}^{-1}\right)^{I J}\left(d \tilde{\zeta}_{I}+(\operatorname{Re} \mathcal{N})_{I K} d \zeta^{K}\right)\left(d \tilde{\zeta}_{J}+(\operatorname{Re} \mathcal{N})_{J L} d \zeta^{L}\right)\right]
\end{aligned}
$$

\footnotetext{
${ }^{21}$ Recall that Cartan's pure spinor of $S O(2 n)$ is isomorphic to $\mathbb{C} \times S O(2 n) / U(n)$.

${ }^{22}$ Recall that $\operatorname{Im} \mathcal{N}$ is a negative-definite, so $\mathcal{M}_{3}$ has split signature $\left(n_{V}+2, n_{V}+2\right)$.
} 
The manifold $\mathcal{M}_{3}^{*}$ is an example of a "para-quaternionic-Kahler manifold" [133]; we shall refer to it as the "c*-map" of the special Kähler manifold $\mathcal{M}_{V}$. This space has isometries

$$
\zeta^{I} \rightarrow \zeta^{I}+\eta^{I}, \quad \tilde{\zeta}_{I} \rightarrow \zeta_{I}+\chi_{I}, \quad a \rightarrow a+\alpha+\chi_{I} \zeta^{I}-\eta^{I} \tilde{\zeta}_{I}
$$

leading to the conserved charges

$$
\begin{aligned}
q_{I} & =-2 e^{-2 U}\left[(\operatorname{Im} \mathcal{N})_{I J} d \zeta^{J}+(\operatorname{Re} \mathcal{N})_{I J}\left(\operatorname{Im} \mathcal{N}^{-1}\right)^{J L}\left(d \tilde{\zeta}_{L}+(\operatorname{Re} \mathcal{N})_{L M} d \zeta^{M}\right)\right]+2 k \tilde{\zeta}_{I} \\
p^{I} & =-2 e^{-2 U}\left(\operatorname{Im} \mathcal{N}^{-1}\right)^{I L}\left(d \tilde{\zeta}_{L}+(\operatorname{Re} \mathcal{N})_{L M} d \zeta^{M}\right)-2 k \zeta^{I} \\
k & =e^{-4 U}\left(d a+\zeta^{I} d \tilde{\zeta}_{I}-\tilde{\zeta}^{I} d \zeta_{I}\right)
\end{aligned}
$$

satisfying the Poisson brackets (7.16). The Hamiltonian corresponding to geodesic motion on $\mathcal{M}_{3}^{*}$ may be rewritten in terms of these charges as

$$
\begin{aligned}
H= & p_{U}^{2}+g^{i \bar{j}^{j}} p_{z^{i}} \bar{p}_{z^{\bar{j}}}-\frac{1}{8} e^{2 U}\left(\operatorname{Im} \mathcal{N}^{-1}\right)_{I K}\left(q_{I}-2 k \tilde{\zeta}_{I}-\mathcal{N}_{I J}\left(p^{J}+2 k \zeta^{J}\right)\right) \\
& \times\left(q_{K}-2 k \tilde{\zeta}_{K}-\overline{\mathcal{N}}_{K L}\left(p^{L}+2 k \zeta^{L}\right)\right)+\frac{1}{2} e^{4 U} k^{2}
\end{aligned}
$$

For vanishing NUT charge $k=0$, the dependence in the axionic scalars $\zeta^{I}, \tilde{\zeta}_{J}$ disappears, and one obtains the Hamiltonian for the radial flow of static black holes in 4 dimensions, which was the starting point for the derivation of the attractor equations in $[40]^{23}$. The general dynamics in the "universal sector" will be discussed in the next Subsection.

In order to find which solutions preserve supersymmetry, we have to determine the supersymmetry variations for a spinning particle propagating on $\mathcal{M}_{3}^{*}$. For this purpose, it is useful to recall some properties of the usual quaternionic-Kähler spaces: such spaces have special holonomy $U S p(2) \times U S p(2 n) \subset S O(4 n)$, and admit three almost complex structures $J^{i}, i=1,2,3$ satisfying the quaternion algebra,

$$
J^{i} \cdot J^{j}=-\delta^{i j}+\epsilon^{i j k} J^{k}
$$

The associated 2-forms $\Omega^{i}(X, Y)=g\left(X, J^{i} Y\right)$ are covariantly constant with respect to a $U S p(2)=S U(2)$ connection $p^{i}$ whose curvature is proportional to $\Omega^{i}$,

$$
d \Omega^{i}+\epsilon^{i j k} p^{j} \wedge \Omega^{k}=0, \quad d p^{i}+\epsilon^{i j k} p^{j} \wedge p^{k}=-i \Omega^{i}
$$

\footnotetext{
${ }^{23}$ In that work, the attractor equations were found by writing $H$ as a sum of squares, and requiring each term to vanish. The derivation we now present has the advantage of making supersymmetry manifest.
} 
The $U S p(2) \times U S p(2 n)$ connection $p+q$ may be obtained from a covariantly constant "quaternionic viel-bein" $V^{\alpha \Gamma}, \alpha=1,2, \Gamma=1, . ., 2 n$ such that

$$
\Omega^{i}=\epsilon_{\alpha \beta}\left(\sigma^{i}\right)_{\gamma}^{\beta} \rho_{\Gamma \Gamma^{\prime}} V^{\alpha \Gamma} \wedge V^{\gamma \Gamma^{\prime}}, \quad d s^{2}=\epsilon_{\alpha \beta} \rho_{\Gamma \Gamma^{\prime}} V^{\alpha \Gamma} \otimes V^{\beta \Gamma^{\prime}}, \quad(d+\Omega) V=0
$$

where $\epsilon_{\alpha \beta}$ and $\rho_{\Gamma \Gamma^{\prime}}$ are the antisymmetric tensors invariant under $U S p(2)$ and $U S p(2 n)$, respectively.

The quaternionic viel-bein enters in the supersymmetric variation of the fermions (7.22). In the case of the $c$-map $\mathcal{M}_{3}$, it was computed in [132]:

$$
V^{\alpha \Gamma}=\left(\begin{array}{cc}
u & v \\
e^{A} & E^{A} \\
-\bar{E}^{A} & \bar{e}^{A} \\
-\bar{v} & \bar{u}
\end{array}\right)
$$

where $e^{A}=e_{i}^{A} d z^{i}$ is a viel-bein of the Special Kähler manifold, $e_{i}^{A} \bar{e}_{A \bar{j}}=g_{i \bar{j}}$, and

$$
\begin{aligned}
u & =e^{K / 2-U} X^{I}\left(d \tilde{\zeta}_{I}+\mathcal{N}_{I J} d \zeta^{J}\right) \\
v & =-d U+\frac{i}{2} e^{-2 U}\left(d a+\zeta^{I} d \tilde{\zeta}_{I}-\tilde{\zeta}^{I} d \zeta_{I}\right) \\
E^{A} & =e^{-U} e_{i}^{A} g^{i \bar{j}} \bar{f}_{\bar{j}}^{I}\left(d \tilde{\zeta}_{I}+\mathcal{N}_{I J} d \zeta^{J}\right)
\end{aligned}
$$

In the case of the $c^{*}$-map, the same results are valid but reality conditions change: $\bar{u}=-u^{*}, \bar{E}=-E^{*}$.

Expressing $d \zeta^{I}, d \tilde{\zeta}_{I}, d a$ in terms of the conserved charges (7.29), the entries in the quaternionic viel-bein may be rewritten as

$$
\begin{aligned}
u & =-\frac{i}{2} e^{K / 2+U} X^{I}\left[q_{I}-2 k \tilde{\zeta}_{I}-\mathcal{N}_{I J}\left(p^{J}+2 k \zeta^{J}\right)\right], \\
v & =-d U+\frac{i}{2} e^{2 U} k \\
e^{A} & =e_{i}^{A} d z^{i} \\
E^{A} & =-\frac{i}{2} e^{U} e^{A i} g^{i \bar{j}} \bar{f}_{\bar{j}}^{I}\left[q_{I}-2 k \tilde{\zeta}_{I}-\mathcal{N}_{I J}\left(p^{J}+2 k \zeta^{J}\right)\right]
\end{aligned}
$$

Now, we return to the supersymmetry variation of the fermions (7.22): the existence of $\epsilon_{\alpha}^{\beta}$ such that $\delta \psi^{A \alpha}$ vanishes implies that the first row of $V$ has to be proportional to the second, hence

$$
\begin{aligned}
-d U+\frac{i}{2} e^{2 U} k & =-\frac{i}{2} e^{i \theta} e^{K / 2+U} X^{I}\left(q_{I}-k \tilde{\zeta}_{I}-\mathcal{N}_{I J}\left(p^{J}+k \zeta^{J}\right)\right) \\
d z^{i} & =-\frac{i}{2} e^{i \theta} e^{U} g^{i \bar{j}} \bar{f}_{\bar{j}}^{I}\left(q_{I}-k \tilde{\zeta}_{I}-\mathcal{N}_{I J}\left(p^{J}+k \zeta^{J}\right)\right)
\end{aligned}
$$


where the phase $\theta$ is determined by requiring that $U$ stays real. These equations may be further simplified by defining the "generalized central charge"

$$
Z(p, q, k)=e^{K / 2}\left[\left(q_{I}-2 k \tilde{\zeta}_{I}\right) X^{I}-\left(p^{I}+2 k \zeta^{I}\right) F_{I}\right]
$$

and generalizing (3.33):

$$
-d U+\frac{i}{2} e^{2 U} k=-\frac{i}{2} e^{i \theta} e^{U} Z, \quad d z^{i}=-i e^{i \theta} \frac{|Z|}{Z} e^{U} g^{i \bar{j}} \partial_{\bar{j}}|Z|
$$

For vanishing NUT charge, we indeed recognize the attractor flow equations (3.27),(3.28). For $k \neq 0$, these equations can also be obtained as a special case of the multi-centered geometries studied in [134].

\subsubsection{The Universal Sector}

At this stage, it is instructive to work out the dynamics in the "universal sector", which encodes the scale $U$, the graviphoton electric and magnetic charges, and the NUT charge $k$. The resulting pseudo-quaternionic-Kähler manifold is the symmetric space $\mathcal{M}_{3}^{*}=S U(2,1) / S l(2) \times U(1)$; it is an analytic continuation of the quaternionicKähler space $\mathcal{M}_{3}=S U(2,1) / S U(2) \times U(1)$, which describes the tree-level couplings of the universal hypermultiplet in 4 dimensions. It is obtained by applying the c-map procedure to the prepotential $F=-i\left(X^{0}\right)^{2}$. The Hamiltonian (excluding the cone direction)

$$
H=\frac{1}{8}\left(p_{U}\right)^{2}-\frac{1}{4} e^{2 U}\left[\left(p_{\tilde{\zeta}}-k \zeta\right)^{2}+\left(p_{\zeta}+k \tilde{\zeta}\right)^{2}\right]+\frac{1}{2} e^{4 U} k^{2}
$$

separates between the $(\tilde{\zeta}, \zeta)$ plane and the $U$ direction (the NUT potential $a$ has been eliminated in favor of its conjugate momentum $k=e^{-4 U}(\dot{a}+\zeta \dot{\tilde{\zeta}}-\tilde{\zeta} \dot{\zeta})$. The potential is depicted on Figure 3. The motion in the $(\tilde{\zeta}, \zeta)$ plane is that of a charged particle in a constant magnetic field. The electric, magnetic charges and the angular momentum $J$ in the plane (not to be confused with that of the black hole, which vanishes by spherical symmetry)

$$
p=p_{\tilde{\zeta}}+\zeta k, \quad q=p_{\zeta}-\tilde{\zeta} k, \quad J=\zeta p_{\tilde{\zeta}}-\tilde{\zeta} p_{\zeta}
$$

satisfy the usual algebra of the Landau problem,

$$
[p, q]=k,[J, p]=q,[J, q]=-p
$$

where $p$ and $q$ are the "magnetic translations". The motion in the $U$ direction is governed effectively by

$$
H=\frac{1}{8}\left(p_{U}\right)^{2}+\frac{1}{2} e^{4 U} k^{2}-\frac{1}{4} e^{2 U}\left[p^{2}+q^{2}-4 k J\right]=C^{2}
$$



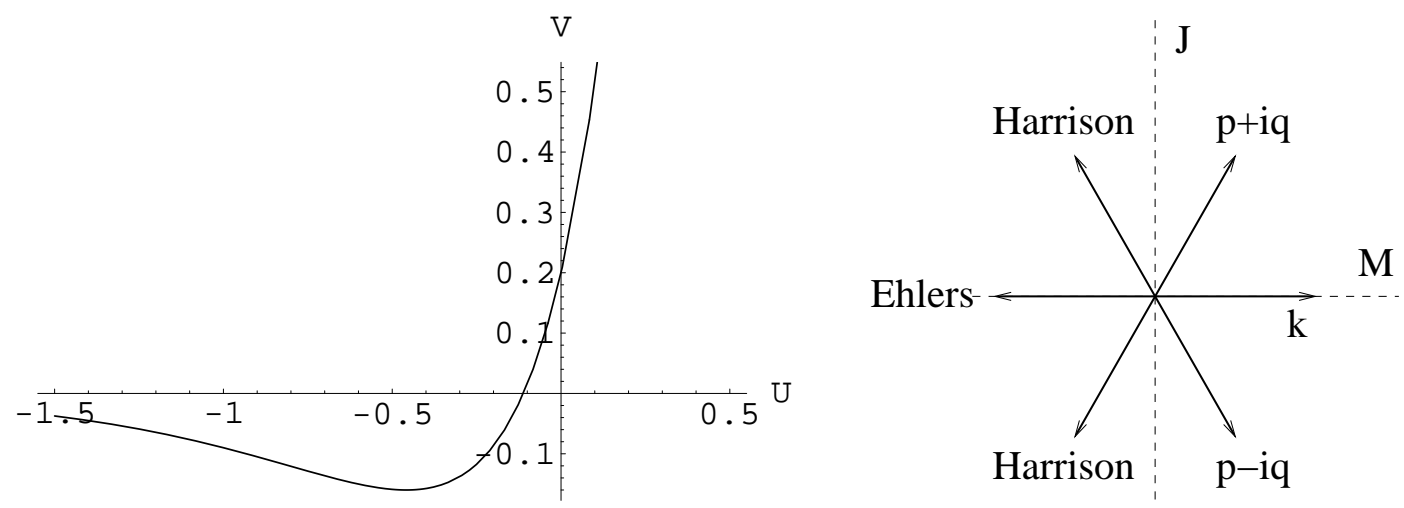

Figure 3: Left: Potential governing the motion along the $U$ variable in the universal sector. The horizon is reached at $U \rightarrow-\infty$. Right: Root diagram of the $S U(2,1)$ symmetries in the universal sector.

At spatial infinity $(\tau=0)$, one may impose the initial conditions $U=\zeta=\tilde{\zeta}=a=0$. The momentum $p_{U}$ at infinity is proportional to the ADM mass, and $J$ vanishes, so the mass shell condition becomes

$$
M^{2}+2 k^{2}-\left(p^{2}+q^{2}\right)=C^{2}
$$

In this simple case, the extremality condition $C^{2}=0$ is equivalent to supersymmetry, since the viel-bein $V$ is a $2 \times 2$ matrix. Equation (7.50) is the BPS mass condition, generalized to non-zero NUT charge. Note that for a given value of $p, q$, there is a maximal value of $k$ such that $M^{2}$ remains positive.

At the horizon $U \rightarrow-\infty, \tau \rightarrow \infty$, the last term in (7.46) is irrelevant, and one may integrate the equation of motion of $U$, and verify that the metric (7.8) becomes $A d S_{2} \times S_{2}$ with area

$$
A=2 \pi\left(p^{2}+q^{2}\right)=2 \pi \sqrt{\left(p^{2}+q^{2}\right)^{2}}
$$

in agreement with the Bekenstein-Hawking entropy of Reissner-Nordström black holes (2.15).

Since the universal sector is a symmetric space, there must exist 3 additional conserved charges, so that the total set of conserved charges can be arranged in an element $Q$ in the Lie algebra $\mathfrak{g}_{3}=s u(2,1)$ (or rather, in its dual $\mathfrak{g}_{3}^{*}$ ). The physical origin of these are the Ehlers and Harrison transformations, which are well known to general relativists. The root diagram of $S U(2,1)$ is depicted on Figure 3. The Casimir invariants of $Q$ are easily computed, and read

$$
\operatorname{Tr}\left(Q^{2}\right)=H, \quad \operatorname{det}(Q)=0
$$


The last condition ensures that the conserved quantities do not overdetermine the motion. The co-adjoint action $Q \rightarrow h Q h^{-1}$ of $G_{3}$ on $\mathfrak{g}_{3}^{*}$ relates different trajectories with the same value of $H$. The phase space, at fixed value of $H$, is therefore a generic co-adjoint orbit of $G_{3}$, of dimension 6 (the symplectic quotient of the full 8-dimensional phase space by the Hamiltonian $H$ ). By the Kirillov-Kostant construction, it carries a canonical symplectic form such that the Noether charges represent the Lie algebra $\mathfrak{g}_{3}$.

As we have just seen, BPS solutions have $H=0$. The standard property of $3 \times 3$ matrices

$$
Q^{3}-\operatorname{Tr}(Q) Q^{2}+\frac{1}{2}\left[\operatorname{Tr}\left(Q^{2}\right)-(\operatorname{Tr} Q)^{2}\right] Q-\operatorname{det}(Q)=0
$$

then implies that $Q^{3}=0$, as a matrix equation in the fundamental representation; more intrinsically, in terms of the adjoint representation, this is equivalent to

$$
[\operatorname{Ad}(Q)]^{5}=0
$$

Thus, $Q$ is a nilpotent element of order 5 in $\mathfrak{g}_{3}^{*}$. This condition is invariant under the coadjoint action of $G_{3}$. We conclude that the classical phase space of BPS configurations is a nilpotent coadjoint orbit ${ }^{24}$ of $G_{3}$. As we shall see, this fact continues to hold in very special gravities where $\mathcal{M}_{3}^{*}$ is a symmetric space.

\subsection{Quantum Attractors}

We now return to general $\mathcal{N}=2$ supergravities, and discuss the radial quantization of stationary, spherically symmetric black holes. As we have seen, the radial evolution equation of the metric, scalar fields and gauge fields in dimension 4 can be recast as the geodesic motion of a fiducial particle on the cone $\mathcal{C}=\mathbb{R}^{+} \times \mathcal{M}_{3}^{*}$.

There is a standard way of quantizing geodesic motion: namely, replace the classical trajectories by square integrable functions wave functions on $\mathcal{C}$, and impose quantum mass-shell condition

$$
\left[-\frac{\partial^{2}}{\partial r^{2}}+\frac{\Delta}{r^{2}}-1\right] \Psi\left(r, \phi^{a}\right)=0
$$

Here $\Delta$ is the Laplace-Beltrami operator on $\mathcal{M}_{3}^{*}$. Since we are dealing with the geodesic motion of a spinning superparticle, we should also represent the fermion operators $\psi^{A \alpha}$ as an element of a Clifford algebra, so that the wave function becomes a section of the Clifford bundle on $\mathcal{C}$, or equivalently a set of differential forms.

Before discussing this more specifically in the context of $\mathcal{N}=2$ supergravities, let us make some comments on the physical meaning of the wave function $\Psi$ :

\footnotetext{
${ }^{24}$ It is a peculiarity of this model that the dimension of this nilpotent orbit is the same -6 - as that of the generic semi-simple orbits. In general, nilpotent orbits can be much smaller than the generic ones.
} 
- As usual in diffeomorphism invariant theories (e.g. quantum cosmology), the wave function is independent of the "time" variable $\rho$, and some other variable should be chosen as a "clock". A natural choice of clock is $r$, as it goes from 0 at the horizon to $\infty$ at spatial infinity. One could also use $e^{U}$, or the black hole area $A=e^{-2 U} r^{2}$ itself, although classically its range depends on the charges

- The wave function is in principle a function of all variables $\phi^{a}$. When $k=0$, one can diagonalize $p^{I}$ and $q_{I}$, simultaneously, which fixes the dependence on $\zeta^{I}, \tilde{\zeta}_{I}$ and $a$. We expect the resulting wave function to be peaked towards the attractor values of the moduli and the horizon area as $r \rightarrow 0$ [135].

- Since (7.55) is a second-order differential equation of Klein-Gordon type, the natural inner product is the Wronskian (or $U(1)$ charge) at a fixed (arbitrary) value of $r$ :

$$
\langle\Psi \mid \Psi\rangle=\int d \phi^{a} \Psi^{*} \stackrel{\leftrightarrow}{\partial_{r}} \Psi
$$

Unfortunately, this product is famously known not to be positive definite. A possible way out is "third quantization" where the wave function $\Psi$ becomes itself an operator [136]. This may be useful in describing the possible black hole fragmentation near the horizon. In the case of spherically symmetric BPS black holes, the extremality constraint $p_{r}=1$ allows to eliminate the radial dependence. We shall therefore drop the radial direction $r$, continue $\mathcal{M}_{3}^{*}$ back to the Riemannian manifold $\mathcal{M}_{3}$, and use the usual $L^{2}$ norm on $\mathcal{M}_{3}$.

\subsubsection{BPS Hilbert space and twistors}

Having described the quantization of general spherically symmetric geometries, we now want to extract the subspace $\mathcal{H}_{\mathrm{BPS}}$ of the full Hilbert space $\mathcal{H}$ which cooresponds to BPS black holes. In this section, we focus on the case of $\mathcal{N}=2$ supersymmetry and give a heuristic derivation which is sufficient for our purposes.

Classically, BPS trajectories are characterized by the existence of a supersymmetry parameter $\epsilon_{\beta}^{\alpha}$ such that $V_{a}^{A \beta} \dot{\phi}^{a} \epsilon_{\beta}^{\alpha}$ vanishes. This constraint may be quantized schematically as

$$
\exists \epsilon / \epsilon_{\beta}^{\alpha} V_{a}^{A \beta} \frac{\partial}{\partial \phi^{a}} \Psi=0
$$

At fixed (projective) $\epsilon$, this implies that the function does not depend on half of the coordinates $X^{A}$. One concludes that $\Psi$ should be a holomorphic function with respect to the complex structure determined by $\epsilon_{\beta}^{\alpha}$. In order to make this statement more precise, let us introduce the twistor space $T$ of the quaternionic-Kähler manifold $\mathcal{M}_{3}$ [137]. This is a $S^{2}$ bundle over $\mathcal{M}_{3}$, which, in contrast to the quaternionic-Kähler base, 
carries a canonical complex (and in fact Kähler) structure. The statement is that $\Psi$ should be a holomorphic section of a line bundle over $T$, more precisely an element of the sheaf cohomology group $H_{l}(T, O(-h))$; the determination of the values $l, h$ would require a better understanding of the fermion zero-mode than we have now. In more mundane terms, the BPS Hilbert space consists of holomorphic functions of $2 n_{V}+1$ variables. As we shall see in the next section, these variables may be thought of as the potentials $\zeta^{I}, \tilde{\zeta}_{I}$ and $a$ conjugate to the electric, magnetic and NUT charge, respectively.

The fact that a special class of massless solutions of the Klein-Gordon equation on $\mathcal{M}_{3}$ can be obtained from certain holomorphic sections on the twistor space $T$ is reminscent of the Penrose - Atiyah - Hitchin - Singer twistor transform in 4 dimensions [138]. In fact, a higher dimensional, quaternionic version of this twistor transform already appears in the math literature [139, 140], and is presumably identical to the one discussed above.

\subsection{Very Special Quantum Attractors}

In preparation for our conjectures about black hole degeneracies and automorphic forms, we now apply this construction to the case of very special $\mathcal{N}=2$ supergravities which we introduced in Section 3.5.

\subsubsection{Quasiconformal Action and Twistor Space}

Recall that the vector-multiplet moduli space of very special supergravities are hermitean symmetric tube domains (3.58), built out of the invariance groups of Jordan algebras $J$ with a cubic norm $N$. The result of the $c$-map ${ }^{25}$ construction [141] is a symmetric space in Alekseevskii's list of quaternionic spaces with transitive solvable isometries [142],

$$
\mathcal{M}_{3}=\frac{\mathrm{Q} \operatorname{Conf}(J)}{\widetilde{\operatorname{Conf}}(J) \times S U(2)}, \quad \mathcal{M}_{3}^{*}=\frac{\mathrm{Q} \operatorname{Conf}(J)}{\operatorname{Conf}(J) \times S l(2)}
$$

where QConf $(J)$ is the "quasi-conformal group" associated to the Jordan algebra $J$ (in its quaternionic, rank 4 real form), and $\widetilde{\operatorname{Conf}}(J)$ is the compact real form of $\operatorname{Conf}(J)$. These spaces can read off from Table 2 on page 25. A two-dimensional projection of the root diagram of $G$ is displayed in Figure 4 (when $J=\mathbb{R}$, this is recognized as the root diagram of $G_{2}$ ).

The group QConf $(J)$ may be defined as the invariance group" ${ }^{26}$ of the "quartic light-cone" $\Delta\left(\Xi, \Xi^{\prime}\right)=0[143]$ where $\Xi$ denotes a triplet $\left(\zeta^{I}, \tilde{\zeta}_{I}, a\right)$ of electric, magnetic

\footnotetext{
${ }^{25}$ The result of the $c^{*}$ map appears in [122].

${ }^{26}$ More precisely, QConf $(J)$ admits an action on $\Xi$ such that, upon acting simultaneously on $\Xi$ and $\Xi^{\prime}, \Delta\left(\Xi, \Xi^{\prime}\right)$ is multiplied by $f(\Xi) f\left(\Xi^{\prime}\right)$
} 


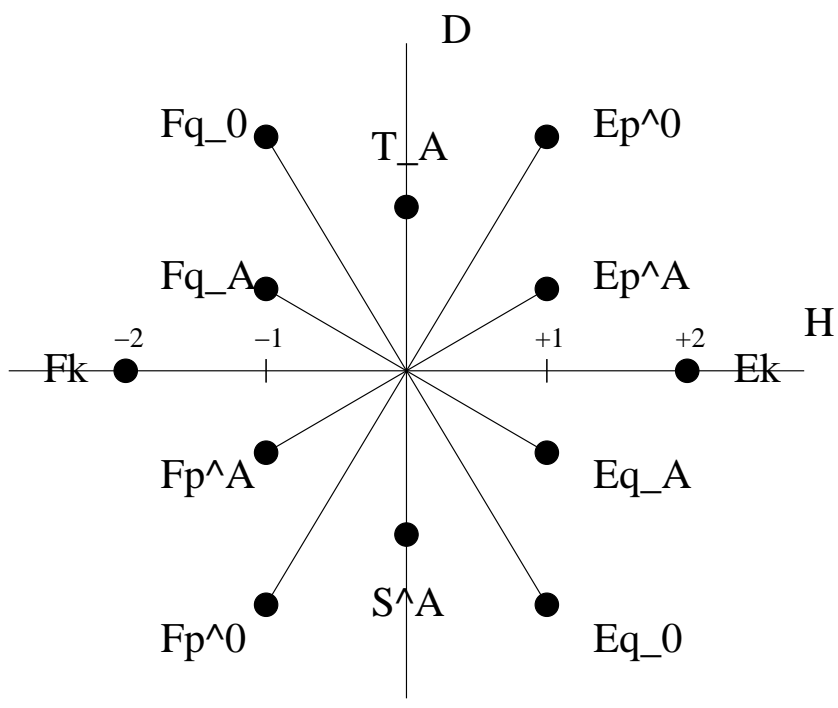

Figure 4: Two-dimensional projection of the root diagram of the quasi-conformal group associated to a cubic Jordan algebra $J$. The five-grading corresponds to the horizontal axis. The long roots are singlets, generating a $S U(2,1)$ universal subgroup, while the short roots are valued in the Jordan algebra $J$.

and NUT potentials, and

$$
\Delta\left(\Xi ; \Xi^{\prime}\right)=I_{4}\left(\zeta^{I}-\zeta^{\prime I}, \tilde{\zeta}_{I}-\tilde{\zeta}_{I}\right)+2\left(a-a^{\prime}+\zeta^{\prime I} \tilde{\zeta}_{I}-\zeta^{I} \tilde{\zeta}_{I}^{\prime}\right)^{2}
$$

where $I_{4}$ is the quartic invariant of $\operatorname{Conf}(J)$. The action of $G=\mathrm{QConf}(J)$ on $\Xi$ follows from the 5-graded decomposition

$$
G=G_{-2} \oplus G_{-1} \oplus G_{0} \oplus G_{+1} \oplus G_{+2}
$$

The top space $G_{+2}$ is one-dimensional, therefore $G_{+1} \oplus G_{+2}$ form an Heisenberg algebra with center $G_{+2}$ : we identify it with the Heisenberg algebra $\left[p^{I}, q_{J}\right]=2 k \delta_{J}^{I}$ of electric, magnetic and NUT isometries (7.16). Symmetrically, the $G_{-1} \oplus G_{-2}$ form an Heisenberg algebra with one-dimensional center $G_{-2}$. The grade 0 space is $G_{0}=\operatorname{Conf}(J) \times U(1)$; Together with $G_{-2}$ and $G_{+2}$, the center $H$ of $G_{0}$ generates an $S U(2)$ subgroup which commutes with $\operatorname{Conf}(J)$, and yields the above 5 -grading above. Finally, $\operatorname{Conf}(J)$ acts linearly on $G_{+1} \sim\left\{p^{I}, q_{I}\right\}$ in the usual way, leaving $G_{+2} \sim\{k\}$ invariant. Since the $H$ charge is additive, the sum $P=G_{-2} \oplus G_{-1} \oplus G_{0}$ closes under commutation, and is known as the Heisenberg parabolic subgroup of $G$. Thus, we may consider the quotient $P \backslash G$, isomorphic to the Heisenberg algebra $G_{+1} \oplus G_{+2} \sim\left(\zeta^{I}, \tilde{\zeta}_{I}, a\right)$ : the action of $G$ on $P \backslash G$ by right multiplication gives the above-mentionned action on $\Xi$. We refer to this action as the "quasiconformal action" of $G$ on $\Xi=\left(\zeta^{I}, \tilde{\zeta}_{I}, a\right)$. This is an exemple of 
the mathematical construction of irreducible representations by unitary induction from the parabolic $P$ to $G$ (see e.g. [144] for a review of this concept): it may be "twisted" by a unitary character $\chi$ of $P$, i.e. by considering functions on $G$ which transform by $\chi$ under the right action of $G$. To be completely explicit, the generators in $G_{+1} \oplus G_{+2}$ act on functions of $\Xi$ as

$$
E_{k}=\partial_{a}, \quad E_{p^{I}}=\partial_{\tilde{\zeta}_{I}}+\zeta^{I} \partial_{a} \quad E_{q_{I}}=\partial_{\zeta^{I}}-\tilde{\zeta}_{I} \partial_{a}
$$

while the generator in $G_{-2}$ acts as

$$
\frac{1}{2}\left(\frac{\partial I_{4}}{\partial \tilde{\zeta}_{I}}-4 a \zeta^{I}\right) \partial_{\zeta^{I}}+\frac{1}{2}\left(-\frac{\partial I_{4}}{\partial \zeta^{I}}-4 a \tilde{\zeta}_{I}\right) \partial_{\tilde{\zeta}_{I}}+\left(I_{4}-2 a^{2}\right) \partial_{a}+4 \nu
$$

where $I_{4}=I_{4}\left(\zeta^{I}, \tilde{\zeta}_{I}\right)$ and $\nu$ is a complex number parametrizing the character $\chi$. The rest of the generators can be obtained by commutation and $\operatorname{Conf}(J)$ rotations. This representation is known as the "quaternionic discrete series" representation of $G$ [145].

By the standard "orbit philosophy" [146], this representation can also be viewed as the quantization of the co-adjoint orbit of a nilpotent element of order 5. Analyzing the BPS equation $V^{A \alpha} \epsilon_{\alpha}^{\beta}=0$ classically, one can show ${ }^{27}$ that the Noether charge for BPS black is also nilpotent of order 5 , generalizing the $S U(2,1)$ result (7.54). This suggests that the quantum Hilbert space for BPS black holes should indeed be the quasi-conformal representation of $G$.

On the other hand, in Section 7.3.1 we argued that the BPS Hilbert space should be given by functions (or rather sections of a line bundle on) the twistor space $T$ on $\mathcal{M}_{3}$. In this case, it is easy to guess what $T$ should look like: it should be a Einstein-Kähler space of complex dimension $2 n_{V}+1$, with an isometric action of QConf $(J)$. There is a natural candidate, namely the space with Kähler potential

$$
K_{T}=-\frac{1}{2} \log \Delta(\Xi, \Xi)
$$

Indeed, we have seen (footnote 26) that $K_{T}$ transforms by Kähler transformations under the quasi-conformal action of $G$ on $\Xi=\left(\zeta^{I}, \tilde{\zeta}_{I}, a\right)$ and its complex conjugate. The formula (7.63) can in fact proven in full generality using projective superspace methods [147]. Thus, holomorphic sections on the twistor space indeed realize the quasi-conformal representation, as suggested by the classical analysis.

We conclude that the BPS Hilbert space $\mathcal{H}_{B P S}$ for the radial quantization of BPS black holes in $\mathcal{N}=2$ supergravity is the representation space of the quasi-conformal representation of the three-dimensional U-duality group.

${ }^{27}$ The BPS equation implies that $V$ can be conjugated in the grade 1 space $G_{+1}$; due to the 5 grading, $[A d(V)]^{5}=0$ is automatic. It remains to observe that the Noether charges $Q$ are in the same conjugacy class with the momentum $V$. 


\subsubsection{Embedding $H_{B P S}$ in $H$ and the Spherical Vector}

In order to extract physical data from the BPS wave function, it is necessary to embed the BPS Hilbert space $\mathcal{H}_{B P S}$ in the Hilbert space of general, non-supersymmetric configurations, $\mathcal{H}$, in other words, to find the "twistor transform" between holomorphic sections of the twistor space $T$ and functions on $\mathcal{M}_{3}$. In the case of very special supergravities, where $\mathcal{M}_{3}$ is a symmetric space $G / K$, this may be done easily once a special vector $f_{K}$ in $\mathcal{H}_{B P S}$, invariant under the maximal compact subgroup $K$, is found: to any vector $f \in \mathcal{H}_{B P S}$ we can then associate the function on $G$

$$
\Psi_{f}(g)=\left\langle f|\rho(g)| f_{K}\right\rangle
$$

Since $f_{K}$ is invariant under $K, \Psi_{f}$ descends to the quotient $G / K$. This construction is standard in representation theory, where $f_{K}$ is referred to as a spherical vector (whereas $\Psi_{f_{K}}$, a special case of (7.64), is referred to as a spherical function) (see again [144]).

The spherical vector in the quasiconformal representation is unique and can be computed explicitely [148]. For the simple case of the universal sector $S U(2,1) / S U(2) \times$ $U(1)$, where the quartic invariant is $I_{4}=\frac{1}{2}\left(\zeta^{2}+\tilde{\zeta}^{2}\right)^{2}$, one may check that

$$
f_{K}(\zeta, \tilde{\zeta}, a)=\left(1+\zeta^{2}+\tilde{\zeta}^{2}+a^{2}+\frac{1}{2} I_{4}\right)^{\nu}
$$

is indeed invariant under $S U(2) \times U(1)$. Acting with $\rho(g)$ where $g$ is parameterized by $U, \zeta^{\prime}, \tilde{\zeta}^{\prime}, a^{\prime}$, one obtains

$$
\left[\rho(g) f_{K}\right](\zeta, \tilde{\zeta}, a)=\left[e^{2 U}+\left(\tilde{\zeta}-\tilde{\zeta}^{\prime}\right)^{2}+\left(\zeta-\zeta^{\prime}\right)^{2}+\frac{1}{4} e^{-2 U} \Delta\left(\zeta, \tilde{\zeta}, a ; \zeta^{\prime}, \tilde{\zeta}^{\prime}, a^{\prime}\right)\right]^{\nu}
$$

Thus, we conclude that BPS wave functions, in the unconstrained Hilbert space $\mathcal{H}$, are given by

$$
\Psi\left(U, \zeta^{\prime}, \tilde{\zeta}^{\prime}, a^{\prime}\right):=\int d \zeta d \tilde{\zeta} d a f^{*}(\zeta, \tilde{\zeta}, a)\left[\rho(g) f_{K}\right]\left(U, \zeta^{\prime}, \tilde{\zeta}^{\prime}, a^{\prime} ; \zeta, \tilde{\zeta}, a\right)
$$

The wave function for a BPS black hole with fixed electric and magnetic charges and zero NUT charge is obtained by substituting $f(\zeta, \tilde{\zeta}, a)=e^{i p \tilde{\xi}+q \zeta}$ in this expression. This computation may be generalized to all very special supergravities, and it may be shown that the wave function is indeed peaked at the attractor value of the 4D moduli [135].

\subsubsection{The Minimal Representation}

We have argued that the radial wave functions for BPS black holes were holomorphic sections of the twistor space $T$, hence holomorphic functions of $2 n_{V}+1$ variables. On 
the other hand, the topological string amplitude, in the real polarization, is a function of $n_{V}$ variables: if the suggestion in [41] is correct, it should then occur in a particular subspace of $\mathcal{H}_{B P S}$.

In fact, it is known in mathematics that the quasiconformal representation, for low values of the parameter $\nu$, is no longer irreducible, nor does it belong to the discrete series any more [145]. This may be understood from the fact that the quasi-conformal action on $\Xi=\left(\zeta^{I}, \tilde{\zeta}_{I}, a\right)$ perserves a certain "flag" decomposition $V \supset X \supset Y \supset Z$, where

$$
V=\left\{\zeta^{I}, \tilde{\zeta}_{I}\right\}, \quad X=\left\{I_{4}=0\right\}, \quad Y=\left\{d I_{4}=0\right\}, \quad Z=\left\{\operatorname{Hess} I_{4}=0\right\}
$$

Here, Hess denotes the projection of the Hessian of $I_{4}$, viewed as an element in $V \otimes_{S} V$, on the adjoint representation of the "four-dimensional U-duality group" $\operatorname{Conf}(J)$. The conditions in (7.68) have a transparent interpretation if one substitutes $\zeta^{I}, \tilde{\zeta}_{I}$ with their conjugate, the black hole charges $p^{I}, q_{I}$ : $V$ corresponds to the generic "large" black hole with 4 independent charges, while $X, Y, Z$ correspond to "small" black holes with 3,2 and 1 charges, respectively. In the context of $\mathcal{N}=8$ supergravity, where $I_{4}$ is the quartic invariant of $E_{7(7)}, M, X, Y, Z$ would be identified as large 1/8-BPS, small 1/8-BPS, small 1/4-BPS and small 1/2-BPS black holes, respectively [149].

It follows that each of these subloci, supplemented by the NUT variable $a$, allows for a representation of $\mathrm{QConf}(J)$, on functions of $2 n_{V}+3,2 n_{V}+2,\left(5 n_{v}+1\right) / 3$ and $n_{V}+2$ variables, respectively. These may be shown to be associated by the "orbit philosophy" to nilpotent co-adjoint orbits of order 5,4,3,2, respectively. In particular, the action of $\mathrm{QConf}(J)$ on functions on $\mathbb{R} \times Z$ is known as the minimal representation of $G$, and plays a distinguished role in mathematics. In physics ${ }^{28}$, it was introduced in $[151,152,153]$ in an attempt to quantize BPS membranes, and in $[154,155]$ by "quantizing the quasi-conformal representation" 29. A recent unified approach can be found in [156].

\subsection{Speculations}

To conclude this chapter, we indulge ourselves in some speculations suggested by our study of the quantum attractor flow for very special supergravities.

\subsubsection{The Generalized Topological String Amplitude}

Comparing the number of variables, $n_{V}+1$, in the real polarized topological string amplitude to the dimension, $n_{V}+2$ of the minimal representation, suggests the existence

\footnotetext{
${ }^{28}$ Early attempts to use the minimal representation of $S l(3)$ for strong interactions go back to [150].

${ }^{29}$ This is somewhat of a misnomer, since the quasi-conformal representation is quantum already.
} 
of a one-parameter generalization of the topological string amplitude, living in the representation space of the minimal representation of $G=\mathrm{QConf}(J)$.

As a first piece of evidence for this claim, the spherical vector of the minimal representation in the split case, computed in [152], reduces to the topological amplitude in a certain limit, at least for the leading cubic part [108]. Preliminary results on the spherical vector for the quaternionic real form confirm this expectation [148].

Moreover, the holomorphic anomaly equations (4.46) obeyed by the usual topological amplitude may be recast in a purely holomorphic form, and shown to follow from the quadratic relations of the "Joseph ideal" which annihilates the minimal representation of $G$, by restriction to the "Fourier-Jacobi group" $P / Z$, where $Z$ is the center of the Heisenberg parabolic [79]. This is in precise analogy with the heat equation satisfied by the classical Jacobi theta series,

$$
\left[i \partial_{\tau}-\partial_{z}^{2}\right] \theta_{1}(\tau, z)=0
$$

which follows from quadratic relations in the minimal (i.e. metaplectic) representation of $S p(4) \supset S l(2)$. In this restriction, the generator $k=G_{+2}$ becomes central and can be fixed to an arbitrary non-zero value, reducing the total number of variables from $n_{V}+2$ down to $n_{V}+1$.

Notice also that the dimension of the minimal representation, $n_{V}+2$ is one quarter of the dimension $4 n_{V}+8$ of the hyperkähler cone over the quaternionic space $\mathrm{QConf}(J) / \operatorname{Conf}(J) \times S U(2)$ (recall for any quaternionic-Kähler space $Q K$ of dimension $4 n_{V}$, one can consider the real cone over the total space of a $S U(2)$ principal bundle over $Q K$; this is a hyperkähler manifold with a $S U(2)$ group of isometries [137]). This suggests that the minimal representation should be viewed as the space of tri-holomorphic functions on the hyperKähler cone $H K C$, acted upon by $G$ (see [157] for background on tri-holomorphic functions). Put together, these facts suggest the following speculations:

a) The usual topological string amplitude $\Psi_{\mathbb{R}}\left(p^{I}\right)$ should arise as a "Fourier-Jacobi" coefficient of a "generalized topological amplitude" $\Psi_{\text {gen }}\left(p^{I}, k\right)$ at $k=1$. It is natural to expect that other values of $k$ would be related to Donaldson-Thomas invariants of ideal sheaves with rank $k$ [79].

b) In Type IIA string theory (and for the A-model topological string), the $k$ dependence of $\Psi_{\text {gen }}$ should capture instanton effects on $\mathcal{M}_{3}$, coming from fourdimensional black holes running around the thermal circle. This seems like a direct route to proving a "modified" OSV conjecture, where $\Psi_{\text {top }}$ would be replaced by $\Psi_{g e n}$. 
c) By T-duality along that circle and decompactification to four dimensions, the same $\Psi_{\text {gen }}$ should capture instanton corrections ${ }^{30}$ to the " $\tilde{F}$-term" interactions (5.10) on the hypermultiplet space of Type IIB string theory compactified on the same Calabi-Yau $X$. Moreover, there should exist an off-shell superspace formulation of these " $\tilde{F}$-term" interactions in terms of a tri-holomorphic function on the hyperkähler cone over the QK space.

d) By virtue of the S-duality of type IIB, or the exchange of the thermal and Mtheory directions on the type IIA side, one expects that $\Psi_{\text {gen }}$ should be a generalized Jacobi form for $S l(2, \mathbb{Z})$. For very special supergravities, it is natural to conjecture that this extends to an invariance of $\Psi_{\text {gen }}$ to the full 3-dimensional U-duality group QConf $(J, \mathbb{Z})$.

\subsubsection{Automorphic Partition Functions}

The last point gives a plausible answer to the question raised in Section 7.1: the black hole radial wave function which, by holography, should capture the exact microscopic degeneracies of BPS black holes in real time quantization, should be a $\operatorname{QConf}(J, \mathbb{Z})$ invariant vector $f_{\mathbb{Z}}$ in $H_{B P S}$. This is a very strong constraint, which, in the case of the minimal representation $H_{\text {min }} \subset H_{B P S}$, is known to have a unique solution. It can be constructed "adelically" by computing the spherical vector over the $p$-adic field $\mathbb{Q Q}_{p}$ and taking the product over all primes $p$, see [144] for a pedestrian introduction to these adelic techniques.

By applying (7.64) with $f=f_{\mathbb{Z}}$, we can produce a function $\theta$ on $G(\mathbb{Z}) \backslash G(\mathbb{R}) / K$, i.e. an automorphic form $\theta$ of $G(\mathbb{Z})$. There is a general prescription for extracting Fourier coefficients labelled by the electric and magnetic charges $q_{I}, p^{I}$ (but not by the NUT charge), namely to integrate over the unipotent radical $N$ of the Heisenberg parabolic subgroup $P$

$$
\hat{\theta}\left(p^{I}, q_{I}\right)=\int_{N(\mathbb{R}) / N(\mathbb{Z})} \xi(g) \theta(g) d g
$$

with a character

$$
\xi_{p, q}=e^{i\left(q_{I} \zeta^{I}+p^{I} \tilde{\zeta}_{I}\right)}
$$

One can show that the result is invariant under the linear action of $\operatorname{Conf}(J, \mathbb{Z})$ on the charges, and has support only on charges with $I_{4}\left(p^{I}, q_{I}\right) \geq 0$ (see e.g. [158] in the case of $\left.G_{2(2)}\right)$. In the case of the small representations associated to $X, Y$ or $Z$, the coefficients

\footnotetext{
${ }^{30}$ The success of the OSV conjecture at large charge requires that the corrections to the hypermultiplet space should factorize as $\Psi_{\text {gen }} \sim\left|\Psi_{\text {top }}\right|^{2}$ in the weak coupling limit. It is unclear yet how this effect could take place.
} 
further vanish outside the corresponding locus. For the minimal representation, the result is easy to calculate,

$$
\hat{\theta}(p, q)=\int d \zeta^{I} e^{i q_{I} \zeta^{I}} f_{\mathbb{Z}}^{*}\left(p^{I}-\zeta^{I}, 0\right) f_{K}\left(p^{I}+\zeta^{I}, 0\right)
$$

and is formally close, yet significantly different, from the OSV conjecture (5.20). According to our conjecture, the coefficients should be identified with the degeneracies of "very small" black holes, which satisfy $\operatorname{Hess}\left(I_{4}\right)\left(p^{I}, q_{I}\right)=0$. Indeed, it is known that Fourier coefficients of the theta function associated to the minimal representation grow as a power of the entropy $\sqrt{I_{4}}$, rather than exponentially [158]. It would be very interesting to construct the automorphic forms attached to the nilpotent orbit of order 5, and see if their Fourier coefficients have the required exponential growth.

\section{Conclusion}

In these lectures, we have reviewed some recent attempts in generalizing the microscopic counting of BPS black holes beyond leading order. Our main emphasis was on the conjecture by Ooguri, Strominger and Vafa, which relates the microscopic degeneracies of four-dimensional BPS black holes to the topological string amplitude, which captures an infinite series of higher-derivative corrections in the macroscopic, low energy theory.

By analyzing the case of "small" black holes, which can be easily counted in the heterotic description, we have found that the topological amplitude captures the microscopic degeneracies with impressive precision. At the same time, it is clear that some kind of non-perturbative generalization of the topological string is required, if one wants to obtain exact agreement for finite charges.

Motivated by the "holographic" interpretation of the OSV conjecture as a channel duality between radial and time-like quantization, we studied the quantization of the attractor flow for stationary, spherically symmetric black holes, by reformulating the attractor flow in terms of a geodesic flow on the moduli space in three-dimensions. While we found a natural habitat for the topological string wave function, the resulting structure is considerably richer than envisaged in [41], and hints at a one-parameter generalization of the usual topological string amplitude. The inclusion of higher F-term interactions, rotating or multi-centered geometries are interesting subjects for further research.

These considerations lend support to the idea that the three-dimensional U-duality group should play a rôle as a spectrum-generating symmetry for 4-dimensional black holes $[149,143,159,48]$. Our framework suggests that the black hole degeneracies 
should be indeed be related to Fourier coefficients of automorphic forms for the threedimensional U-duality group $G$, attached to the representations of $G$ which appear in the radial quantization of stationary, spherically symmetric BPS black holes. However, the corresponding automorphic forms have yet to be constructed.

The most obvious application is to BPS black holes in $\mathcal{N}=4$ and $\mathcal{N}=8$ string theory: in this case, the three-dimensional U-duality groups are $S O(8,24, \mathbb{Z})$ and $E_{8(8)}(\mathbb{Z})$, respectively. In the $\mathcal{N}=4$ case, our proposal thus differs from the DVV formula, which relies on an automorphic form of $S p(4, \mathbb{Z})$. It is interesting to remark that $S p(4)$ can be viewed as a "degeneration" of the three- dimensional U-duality group QConf $(J)$ (for any $J$ ), upon collapsing all electric and magnetic charges $p^{I}$ and $q_{I}$ to just two charges $p, q$. This indicates that our proposal may be able to resolve differences between black holes which have the same continuous U-duality invariant, but sit in different orbits of the discrete U-duality group. It will be interesting to see if agreement with the DVV formula is obtained at least for certain orbits.

Exercice 20 Show that the root diagram of $S p(4)$ is "tic-tac-toe"-shaped. Compare to the root diagram of $Q C \operatorname{Conf}(J)$ in Figure 4 on page 71.

Finally, we believe that some automorphic structure could be present in general Calabi-Yau compactifications (even though the moduli space is not a symmetric space), relying on the monodromy group of $X$. It would be very interesting to unravel this arithmetic structure.

\section{Acknowledgements}

These notes represent the content of lectures delivered at the RTN Winter School on Strings, Supergravity and Gauge theories, (CERN, January 16-20, 2006), the 11-th APCTP/KIAS String Winter School (Pohang, Feb 8-15 2005) and the Winter School on the Attractor Mechanism (Frascati, March 20-24, 2006). I am very grateful to the respective organizers for inviting me to give these lectures, and to the participants for their interest and questions. I also wish to thank A. Dabholkar, F. Denef, M. Gunaydin, G. Moore, A. Neitzke and A. Waldron for an enjoyable collaboration leading to the results presented in Sections 6 and 7, and I. Bena, S. Belluci, B. de Wit, R. Dijkgraaf, E. Gimon, R. Kallosh, A. Keurentjes, P. Kraus, M. Marino, S. Miller, M. Rocek, S. Vandoren, E. Verlinde for useful discussions on the some of the material covered in these lectures. The hospitality of CIRM, Luminy during the final write-up of these notes is kindly acknowledged. This research is supported in part by the EU under contracts MTRN-CT-2004-005104, MTRN-CT-2004-512194, and by ANR (CNRSUSAR) contract No 05-BLAN-0079-01. 


\section{References}

[1] S. A. Hughes, "Trust but verify: The case for astrophysical black holes," ECONF C0507252 (2005) L006, hep-ph/0511217.

[2] T. Banks, "A critique of pure string theory: Heterodox opinions of diverse dimensions," hep-th/0306074.

[3] P. K. Townsend, "Black holes," gr-qc/9707012.

[4] T. Damour, "The entropy of black holes: A primer," hep-th/0401160.

[5] S. W. Hawking, "Particle creation by black holes," Commun. Math. Phys. 43 (1975) 199-220.

[6] W. G. Unruh, "Notes on black hole evaporation," Phys. Rev. D14 (1976) 870.

[7] R. Wald, "Black hole thermodynamics," Living Rev. Relativity 4 (2001) http://www. livingreviews.org/lrr-2001-6.

[8] A. Strominger, "Ads(2) quantum gravity and string theory," JHEP 01 (1999) 007, hep-th/9809027.

[9] E. Witten and D. I. Olive, "Supersymmetry algebras that include topological charges," Phys. Lett. B78 (1978) 97.

[10] P. K. Tripathy and S. P. Trivedi, "Non-supersymmetric attractors in string theory," JHEP 03 (2006) 022, hep-th/0511117.

[11] K. Goldstein, N. Iizuka, R. P. Jena, and S. P. Trivedi, "Non-supersymmetric attractors," Phys. Rev. D72 (2005) 124021, hep-th/0507096.

[12] R. Kallosh, "New attractors," JHEP 12 (2005) 022, hep-th/0510024.

[13] B. Sahoo and A. Sen, "Higher derivative corrections to non-supersymmetric extremal black holes in $\mathrm{n}=2$ supergravity," hep-th/0603149.

[14] N. Arkani-Hamed, L. Motl, A. Nicolis, and C. Vafa, "The string landscape, black holes and gravity as the weakest force," hep-th/0601001.

[15] Y. Kats, L. Motl, and M. Padi, "Higher-order corrections to mass-charge relation of extremal black holes," hep-th/0606100.

[16] J. M. Maldacena, "Black holes in string theory," hep-th/9607235.

[17] A. W. Peet, "Tasi lectures on black holes in string theory," hep-th/0008241. 
[18] J. R. David, G. Mandal, and S. R. Wadia, "Microscopic formulation of black holes in string theory," Phys. Rept. 369 (2002) 549-686, hep-th/0203048.

[19] S. D. Mathur, "The quantum structure of black holes," Class. Quant. Grav. 23 (2006) R115, hep-th/0510180.

[20] A. Strominger and C. Vafa, "Microscopic origin of the Bekenstein-Hawking entropy," Phys. Lett. B379 (1996) 99-104, hep-th/9601029.

[21] J. M. Maldacena and A. Strominger, "Statistical entropy of four-dimensional extremal black holes," Phys. Rev. Lett. 77 (1996) 428-429, hep-th/9603060.

[22] C. V. Johnson, R. R. Khuri, and R. C. Myers, "Entropy of 4d extremal black holes," Phys. Lett. B378 (1996) 78-86, hep-th/9603061.

[23] J. M. Maldacena, A. Strominger, and E. Witten, "Black hole entropy in M-theory," JHEP 12 (1997) 002, hep-th/9711053.

[24] R. Dijkgraaf, H. L. Verlinde, and E. P. Verlinde, "Counting dyons in $\mathcal{N}=4$ string theory," Nucl. Phys. B484 (1997) 543-561, hep-th/9607026.

[25] M. Cvetic and A. A. Tseytlin, "Solitonic strings and bps saturated dyonic black holes," Phys. Rev. D53 (1996) 5619-5633, hep-th/9512031.

[26] G. L. Cardoso, B. de Wit, J. Kappeli, and T. Mohaupt, "Asymptotic degeneracy of dyonic $\mathrm{N}=4$ string states and black hole entropy," hep-th/0412287.

[27] D. Shih, A. Strominger, and X. Yin, "Recounting dyons in $\mathcal{N}=4$ string theory," hep-th/0505094.

[28] D. P. Jatkar and A. Sen, "Dyon spectrum in chl models," JHEP 04 (2006) 018, hep-th/0510147.

[29] J. R. David, D. P. Jatkar, and A. Sen, "Product representation of dyon partition function in chl models," hep-th/0602254.

[30] A. Dabholkar and S. Nampuri, "Spectrum of dyons and black holes in chl orbifolds using borcherds lift," hep-th/0603066.

[31] D. Gaiotto, "Re-recounting dyons in $\mathrm{n}=4$ string theory," hep-th/0506249.

[32] A. Ceresole, R. D'Auria, and S. Ferrara, "The symplectic structure of n=2 supergravity and its central extension," Nucl. Phys. Proc. Suppl. 46 (1996) 67-74, hep-th/9509160. 
[33] P. Fre, "Supersymmetry and first order equations for extremal states: Monopoles, hyperinstantons, black holes and p- branes," Nucl. Phys. Proc. Suppl. 57 (1997) 52-64, hep-th/9701054.

[34] G. W. Moore, "Arithmetic and attractors," hep-th/9807087.

[35] T. Mohaupt, "Black hole entropy, special geometry and strings," Fortsch. Phys. 49 (2001) 3-161, hep-th/0007195.

[36] K. Hori, S. Katz, A. Klemm, R. Pandharipande, R. Thomas, C. Vafa, R. Vakil, and E. Zaslow, Mirror symmetry, vol. 1 of Clay Mathematics Monographs. American Mathematical Society, Providence, RI, 2003. With a preface by Vafa.

[37] M. Huebscher, P. Meessen, and T. Ortin, "Supersymmetric solutions of $n=2 d=4$ sugra: the whole ungauged shebang," hep-th/0606281.

[38] S. Ferrara, R. Kallosh, and A. Strominger, “N $=2$ extremal black holes," Phys. Rev. D52 (1995) 5412-5416, hep-th/9508072.

[39] S. Ferrara and R. Kallosh, "Universality of supersymmetric attractors," Phys. Rev. D54 (1996) 1525-1534, hep-th/9603090.

[40] S. Ferrara, G. W. Gibbons, and R. Kallosh, "Black holes and critical points in moduli space," Nucl. Phys. B500 (1997) 75-93, hep-th/9702103.

[41] H. Ooguri, C. Vafa, and E. P. Verlinde, "Hartle-Hawking wave-function for flux compactifications," hep-th/0502211.

[42] G. Lopes Cardoso, B. de Wit, J. Kappeli, and T. Mohaupt, "Black hole partition functions and duality," JHEP 03 (2006) 074, hep-th/0601108.

[43] M. Gunaydin, G. Sierra, and P. K. Townsend, "The geometry of $\mathcal{N}=2$ Maxwell-Einstein supergravity and Jordan algebras," Nucl. Phys. B242 (1984) 244.

[44] K. McCrimmon, "Jordan algebras and their applications," Bull. Amer. Math. Soc. 84 (1978), no. 4, 612-627.

[45] P. Jordan, J. von Neumann, and E. P. Wigner, "On an algebraic generalization of the quantum mechanical formalism," Annals Math. 35 (1934) 29-64.

[46] M. Gunaydin, G. Sierra, and P. K. Townsend, "Exceptional supergravity theories and the magic square," Phys. Lett. B133 (1983) 72.

[47] S. Ferrara, E. G. Gimon, and R. Kallosh, "Magic supergravities, $\mathrm{n}=8$ and black hole composites," hep-th/0606211. 
[48] B. Pioline, "BPS black hole degeneracies and minimal automorphic representations," JHEP 0508 (2005) 071, hep-th/0506228.

[49] D. Etingof P., Kazhdan and A. Polishchuk, "When is the Fourier transform of an elementary function elementary ?," math.AG/0003009.

[50] B. Pioline, "Cubic free field theory," hep-th/0302043.

[51] M. J. Duff, "String triality, black hole entropy and cayley's hyperdeterminant," hep-th/0601134.

[52] R. Kallosh and A. Linde, "Strings, black holes, and quantum information," Phys. Rev. D73 (2006) 104033, hep-th/0602061.

[53] R. Kallosh and B. Kol, "e $e_{7}$ symmetric area of the black hole horizon," Phys. Rev. D53 (1996) 5344-5348, hep-th/9602014.

[54] D. Gaiotto, A. Strominger, and X. Yin, "New connections between 4d and 5d black holes," JHEP 02 (2006) 024, hep-th/0503217.

[55] E. Witten, "Mirror manifolds and topological field theory," hep-th/9112056.

[56] M. Marino, "Chern-simons theory and topological strings," Rev. Mod. Phys. 77 (2005) 675-720, hep-th/0406005.

[57] M. Marino, "Les houches lectures on matrix models and topological strings," hep-th/0410165.

[58] A. Neitzke and C. Vafa, "Topological strings and their physical applications," hep-th/0410178.

[59] M. Vonk, "A mini-course on topological strings," hep-th/0504147.

[60] S. Cordes, G. W. Moore, and S. Ramgoolam, "Lectures on 2-d yang-mills theory, equivariant cohomology and topological field theories," Nucl. Phys. Proc. Suppl. 41 (1995) 184-244, hep-th/9411210.

[61] M. Bershadsky, S. Cecotti, H. Ooguri, and C. Vafa, "Kodaira-Spencer theory of gravity and exact results for quantum string amplitudes," Commun. Math. Phys. 165 (1994) 311-428, hep-th/9309140.

[62] R. Gopakumar and C. Vafa, "M-theory and topological strings. I," hep-th/9809187.

[63] R. Gopakumar and C. Vafa, "M-theory and topological strings. II," hep-th/9812127.

[64] A. Dabholkar, F. Denef, G. W. Moore, and B. Pioline, "Precision counting of small black holes," JHEP 10 (2005) 096, hep-th/0507014. 
[65] M. T. Grisaru, A. E. M. van de Ven, and D. Zanon, "Four loop divergences for the $\mathrm{n}=1$ supersymmetric nonlinear sigma model in two-dimensions," Nucl. Phys. B277 (1986) 409.

[66] P. Candelas, X. C. De La Ossa, P. S. Green, and L. Parkes, "A pair of Calabi-Yau manifolds as an exactly soluble superconformal theory," Nucl. Phys. B359 (1991) $21-74$.

[67] I. Antoniadis, S. Ferrara, R. Minasian, and K. S. Narain, " $R^{4}$ couplings in M- and type II theories on Calabi-Yau spaces," Nucl. Phys. B507 (1997) 571-588, hep-th/9707013.

[68] M. Marino and G. W. Moore, "Counting higher genus curves in a Calabi-Yau manifold," Nucl. Phys. B543 (1999) 592-614, hep-th/9808131.

[69] C. Faber and R. Pandharipande, "Hodge integrals and Gromov-Witten theory," math. AG/9810173.

[70] A. Dabholkar, F. Denef, G. W. Moore, and B. Pioline, "Exact and asymptotic degeneracies of small black holes," JHEP 08 (2005) 021, hep-th/0502157.

[71] R. P. Thomas, "Gauge theories on Calabi-Yau manifolds," 1997. Available as of July 2006 at http://www.ma.ic.ac.uk/ rpwt/thesis.pdf.

[72] N. A. Nekrasov, H. Ooguri, and C. Vafa, "S-duality and topological strings," hep-th/0403167.

[73] A. Kapustin, "Gauge theory, topological strings, and S-duality," JHEP 09 (2004) 034, hep-th/0404041.

[74] D. Maulik, N. A. Nekrasov, A. Okounkov, and R. Pandharipande, "Gromov-Witten theory and Donaldson-Thomas theory," math.AG/0312059.

[75] D. Maulik, N. A. Nekrasov, A. Okounkov, and R. Pandharipande, "Gromov-Witten theory and Donaldson-Thomas theory, II," math.AG/0406092.

[76] A. Okounkov and R. Pandharipande, "The local Donaldson-Thomas theory of curves," math.AG/0512573.

[77] E. Verlinde, "Attractors and the holomorphic anomaly," hep-th/0412139.

[78] E. Witten, "Quantum background independence in string theory," hep-th/9306122.

[79] M. Gunaydin, A. Neitzke, and B. Pioline, "Topological wave functions and heat equations," hep-th/0607200. 
[80] E. D'Hoker and D. H. Phong, "Complex geometry and supergeometry," hep-th/0512197.

[81] N. Berkovits, "Ictp lectures on covariant quantization of the superstring," hep-th/0209059.

[82] I. Antoniadis, E. Gava, K. S. Narain, and T. R. Taylor, "Topological amplitudes in string theory," Nucl. Phys. B413 (1994) 162-184, hep-th/9307158.

[83] I. Antoniadis, E. Gava, K. S. Narain, and T. R. Taylor, "N=2 type II heterotic duality and higher derivative F terms," Nucl. Phys. B455 (1995) 109-130, hep-th/9507115.

[84] G. Lopes Cardoso, B. de Wit, and T. Mohaupt, "Corrections to macroscopic supersymmetric black-hole entropy," Phys. Lett. B451 (1999) 309-316, hep-th/9812082.

[85] G. Lopes Cardoso, B. de Wit, and T. Mohaupt, "Deviations from the area law for supersymmetric black holes," Fortsch. Phys. 48 (2000) 49-64, hep-th/9904005.

[86] G. Lopes Cardoso, B. de Wit, and T. Mohaupt, "Macroscopic entropy formulae and non-holomorphic corrections for supersymmetric black holes," Nucl. Phys. B567 (2000) 87-110, hep-th/9906094.

[87] G. Lopes Cardoso, B. de Wit, and T. Mohaupt, "Area law corrections from state counting and supergravity," Class. Quant. Grav. 17 (2000) 1007-1015, hep-th/9910179.

[88] R. M. Wald, "Black hole entropy in the Noether charge," Phys. Rev. D48 (1993) 3427-3431, gr-qc/9307038.

[89] P. Kraus and F. Larsen, "Microscopic black hole entropy in theories with higher derivatives," hep-th/0506176.

[90] H. Ooguri, A. Strominger, and C. Vafa, "Black hole attractors and the topological string," Phys. Rev. D70 (2004) 106007, hep-th/0405146.

[91] A. Sen, "Black hole entropy function and the attractor mechanism in higher derivative gravity," JHEP 09 (2005) 038, hep-th/0506177.

[92] C. Vafa, "Two dimensional Yang-Mills, black holes and topological strings," hep-th/0406058.

[93] M. Aganagic, H. Ooguri, N. Saulina, and C. Vafa, "Black holes, q-deformed 2d Yang-Mills, and non-perturbative topological strings," hep-th/0411280. 
[94] D. J. Gross and I. Taylor, Washington, "Two-dimensional qcd is a string theory," Nucl. Phys. B400 (1993) 181-210, hep-th/9301068.

[95] R. Dijkgraaf, R. Gopakumar, H. Ooguri, and C. Vafa, "Baby universes in string theory," hep-th/0504221.

[96] D. Shih and X. Yin, "Exact black hole degeneracies and the topological string," JHEP 04 (2006) 034, hep-th/0508174.

[97] D. Gaiotto, A. Strominger, and X. Yin, "From ads(3)/cft(2) to black holes / topological strings," hep-th/0602046.

[98] F. Denef and G. W. Moore. To appear.

[99] A. Dabholkar, "Exact counting of black hole microstates," hep-th/0409148.

[100] A. Dabholkar and J. A. Harvey, "Nonrenormalization of the superstring tension," Phys. Rev. Lett. 63 (1989) 478.

[101] A. Dabholkar, G. W. Gibbons, J. A. Harvey, and F. Ruiz Ruiz, "Superstrings and solitons," Nucl. Phys. B340 (1990) 33-55.

[102] R. Dijkgraaf, J. M. Maldacena, G. W. Moore, and E. Verlinde, "A black hole Farey tail," hep-th/0005003.

[103] J. A. Harvey and G. W. Moore, "Fivebrane instantons and $R^{2}$ couplings in $\mathrm{N}=4$ string theory," Phys. Rev. D57 (1998) 2323-2328, hep-th/9610237.

[104] A. Dabholkar, R. Kallosh, and A. Maloney, "A stringy cloak for a classical singularity," JHEP 12 (2004) 059, hep-th/0410076.

[105] V. Hubeny, A. Maloney, and M. Rangamani, "String-corrected black holes," hep-th/0411272.

[106] A. Sen, "Black holes and the spectrum of half-bps states in $\mathrm{n}=4$ supersymmetric string theory," Adv. Theor. Math. Phys. 9 (2005) 527-558, hep-th/0504005.

[107] A. Sen, "Black holes, elementary strings and holomorphic anomaly," JHEP 07 (2005) 063, hep-th/0502126.

[108] M. Gunaydin, A. Neitzke, B. Pioline, and A. Waldron, "Bps black holes, quantum attractor flows and automorphic forms," Phys. Rev. D73 (2006) 084019, hep-th/0512296.

[109] H. A. Kastrup and T. Thiemann, "Canonical quantization of spherically symmetric gravity in Ashtekar's selfdual representation," Nucl. Phys. B399 (1993) 211-258, gr-qc/9310012. 
[110] K. V. Kuchar, "Geometrodynamics of Schwarzschild black holes," Phys. Rev. D50 (1994) 3961-3981, gr-qc/9403003.

[111] M. Cavaglia, V. de Alfaro, and A. T. Filippov, "Hamiltonian formalism for black holes and quantization," Int. J. Mod. Phys. D4 (1995) 661-672, gr-qc/9411070.

[112] H. Hollmann, "Group theoretical quantization of Schwarzschild and Taub-NUT," Phys. Lett. B388 (1996) 702-706, gr-qc/9609053.

[113] H. Hollmann, "A harmonic space approach to spherically symmetric quantum gravity," gr-qc/9610042.

[114] P. Breitenlohner, H. Hollmann, and D. Maison, "Quantization of the Reissner-Nordström black hole," Phys. Lett. B432 (1998) 293-297, gr-qc/9804030.

[115] G. Mandal, "Fermions from half-bps supergravity," JHEP 08 (2005) 052, hep-th/0502104.

[116] L. Maoz and V. S. Rychkov, "Geometry quantization from supergravity: The case of 'bubbling ads'," JHEP 08 (2005) 096, hep-th/0508059.

[117] L. Grant, L. Maoz, J. Marsano, K. Papadodimas, and V. S. Rychkov, "Minisuperspace quantization of 'bubbling ads' and free fermion droplets," JHEP $\mathbf{0 8}$ (2005) 025, hep-th/0505079.

[118] I. Biswas, D. Gaiotto, S. Lahiri, and S. Minwalla, "Supersymmetric states of $\mathrm{n}=4$ yang-mills from giant gravitons," hep-th/0606087.

[119] V. S. Rychkov, "D1-d5 black hole microstate counting from supergravity," JHEP 01 (2006) 063, hep-th/0512053.

[120] J. M. Maldacena, J. Michelson, and A. Strominger, "Anti-de Sitter fragmentation," JHEP 02 (1999) 011, hep-th/9812073.

[121] B. Pioline and J. Troost, "Schwinger pair production in $\operatorname{ads}(2)$," JHEP 03 (2005) 043, hep-th/0501169.

[122] P. Breitenlohner, G. W. Gibbons, and D. Maison, "Four-dimensional black holes from Kaluza-Klein theories," Commun. Math. Phys. 120 (1988) 295.

[123] C. M. Hull and B. L. Julia, "Duality and moduli spaces for time-like reductions," Nucl. Phys. B534 (1998) 250-260, hep-th/9803239.

[124] T. Damour, M. Henneaux, and H. Nicolai, "Cosmological billiards," Class. Quant. Grav. 20 (2003) R145-R200, hep-th/0212256. 
[125] B. Pioline and A. Waldron, "Quantum cosmology and conformal invariance," Phys. Rev. Lett. 90 (2003) 031302, hep-th/0209044.

[126] V. de Alfaro, S. Fubini, and G. Furlan, "Conformal invariance in quantum mechanics," Nuovo Cim. A34 (1976) 569.

[127] R. Kallosh, N. Sivanandam, and M. Soroush, "The non-bps black hole attractor equation," JHEP 03 (2006) 060, hep-th/0602005.

[128] N. A. Obers and B. Pioline, "U-duality and M-theory," Phys. Rept. 318 (1999) 113-225, hep-th/9809039.

[129] J. Sekiguchi, "Remarks on real nilpotent orbits of a symmetric pair," J. Math. Soc. Japan 39 (1987), no. 1, 127-138.

[130] M. Vergne, "Instantons et correspondance de Kostant-Sekiguchi," C. R. Acad. Sci. Paris Sér. I Math. 320 (1995), no. 8, 901-906.

[131] S. Cecotti, S. Ferrara, and L. Girardello, "Geometry of type II superstrings and the moduli of superconformal field theories," Int. J. Mod. Phys. A4 (1989) 2475.

[132] S. Ferrara and S. Sabharwal, "Quaternionic manifolds for type II superstring vacua of Calabi-Yau spaces," Nucl. Phys. B332 (1990) 317.

[133] V. Cortes, C. Mayer, T. Mohaupt, and F. Saueressig, "Special geometry of euclidean supersymmetry. ii: Hypermultiplets and the c-map," JHEP 06 (2005) 025, hep-th/0503094.

[134] F. Denef, "Supergravity flows and d-brane stability," JHEP 08 (2000) 050, hep-th/0005049.

[135] M. Gunaydin, A. Neitzke, B. Pioline, and A. Waldron, "Quantum attractors." To appear.

[136] S. B. Giddings and A. Strominger, "Baby universes, third quantization and the cosmological constant," Nucl. Phys. B321 (1989) 481.

[137] B. de Wit, M. Rocek, and S. Vandoren, "Hypermultiplets, hyperkaehler cones and quaternion-kaehler geometry," JHEP 02 (2001) 039, hep-th/0101161.

[138] M. F. Atiyah, N. J. Hitchin, and I. M. Singer, "Self-duality in four-dimensional Riemannian geometry," Proc. Roy. Soc. London Ser. A 362 (1978), no. 1711, 425-461.

[139] S. M. Salamon, "Differential geometry of quaternionic manifolds," Annales Scientifiques de l'École Normale Supérieure Sr. 4, 19 (1986) 31-55.

[140] R. J. Baston, "Quaternionic complexes," J. Geom. Phys. 8 (1992), no. 1-4, 29-52. 
[141] S. Cecotti, "Homogeneous kahler manifolds and t algebras in $\mathrm{n}=2$ supergravity and superstrings," Commun. Math. Phys. 124 (1989) 23-55.

[142] D. V. Alekseevskiū, "Classification of quaternionic spaces with transitive solvable group of motions," Izv. Akad. Nauk SSSR Ser. Mat. 39 (1975), no. 2, 315-362, 472.

[143] M. Gunaydin, K. Koepsell, and H. Nicolai, "Conformal and quasiconformal realizations of exceptional Lie groups," Commun. Math. Phys. 221 (2001) 57-76, hep-th/0008063.

[144] B. Pioline and A. Waldron, "Automorphic forms: A physicist's survey," hep-th/0312068.

[145] B. H. Gross and N. R. Wallach, "On quaternionic discrete series representations, and their continuations," J. Reine Angew. Math. 481 (1996) 73-123.

[146] A. A. Kirillov, "Merits and demerits of the orbit method," Bull. Amer. Math. Soc. (N.S.) 36 (1999), no. 4, 433-488.

[147] M. Günaydin, B. Pioline, S. Vandoren, and M. Rocek. To appear.

[148] M. Gunaydin, A. Neitzke, O. Pavlyk, B. Pioline, and A. Waldron, "Quasiconformal, minimal representations and their spherical vectors." To appear.

[149] S. Ferrara and M. Gunaydin, "Orbits of exceptional groups, duality and BPS states in string theory," Int. J. Mod. Phys. A13 (1998) 2075-2088, hep-th/9708025.

[150] L. C. Biedenharn, R. Y. Cusson, M. Y. Han, and O. L. Weaver, "Hadronic regge sequences as primitive realizations of sl(3,r) symmetry," Phys. Lett. B42 (1972) $257-260$.

[151] B. Pioline, H. Nicolai, J. Plefka, and A. Waldron, " $R^{4}$ couplings, the fundamental membrane and exceptional theta correspondences," JHEP 03 (2001) 036, hep-th/0102123.

[152] D. Kazhdan, B. Pioline, and A. Waldron, "Minimal representations, spherical vectors, and exceptional theta series. I," Commun. Math. Phys. 226 (2002) 1-40, hep-th/0107222.

[153] B. Pioline and A. Waldron, "The automorphic membrane," JHEP 06 (2004) 009, hep-th/0404018.

[154] M. Gunaydin, K. Koepsell, and H. Nicolai, "The minimal unitary representation of $E_{8(8)}, "$ Adv. Theor. Math. Phys. 5 (2002) 923-946, hep-th/0109005. 
[155] M. Gunaydin and O. Pavlyk, "Minimal unitary realizations of exceptional U-duality groups and their subgroups as quasiconformal groups," JHEP 01 (2005) 019, hep-th/0409272.

[156] M. Gunaydin and O. Pavlyk, "A unified approach to the minimal unitary realizations of noncompact groups and supergroups," hep-th/0604077.

[157] D. Anselmi and P. Fre, "Topological sigma models in four-dimensions and triholomorphic maps," Nucl. Phys. B416 (1994) 255-300, hep-th/9306080.

[158] W. T. Gan, B. H. Gross, and G. Savin, "Fourier coefficients of modular forms on $G_{2}$," Duke Math. J. 115 (2002), no. 1, 105-169.

[159] M. Gunaydin, "Unitary realizations of U-duality groups as conformal and quasiconformal groups and extremal black holes of supergravity theories," AIP Conf. Proc. 767 (2005) 268-287, hep-th/0502235. 